\title{
Simultaneous Nonvanishing of Twists of Automorphic L-Functions
}

\author{
P. MICHEL, ${ }^{1, \star}$ and J. VANDERKAM ${ }^{2}$ \\ ${ }^{1}$ Mathématiques, Université Montpellier II, CC 051, 34095, Montpellier Cedex 05, France. \\ e-mail:michel@darboux.math.univ-montp2.fr \\ ${ }^{2}$ Center for Communications Research, Thanet Road, Princeton, NJ 08540, U.S.A. \\ e-mail:vanderkm@idaccr.org
}

(Received: 30 December 2000; accepted in final form: 13 September 2001)

\begin{abstract}
Given three distinct primitive complex characters $\chi_{1}, \chi_{2}, \chi_{3}$ satisfying some technical conditions, we prove that the triple product of twisted $L$-functions $L\left(f \cdot \chi_{1}, 1 / 2\right) L\left(f \cdot \chi_{2}, 1 / 2\right)$ $L\left(f \cdot \chi_{3}, 1 / 2\right)$ does not vanish for a positive proportion of weight 2 primitive forms for $\Gamma_{0}(q)$, when $q$ goes to infinity through the set of prime numbers. This result, together with some variants, implies the existence of quotients of $J_{0}(q)$ of large dimension satisfying the Birch-Swinnerton-Dyer conjecture over cyclic number fields of degree less than 5.
\end{abstract}

Mathematics Subject Classifications (2000). Primary: 11F66; Secondary: 11M41, 11G40.

Key words. automorphic $L$-functions, central values, mollification.

\section{Introduction}

In recent years, questions about nonvanishing of automorphic $L$-functions at their critical point have received considerable attention [BFH, Du, Lu1, MM, IS, PS]. One reason for this is their connections with topics such as the Phillips-Sarnak deformation theory of Maass forms, the conjecture of Birch and Swinnerton-Dyer, and the theory of liftings of automorphic forms.

This work deals with the nonvanishing for central values of $L$ functions attached to the family $S_{2}^{*}(q)$ of primitive Hecke eigenforms forms of weight 2, for the group $\Gamma_{0}(q)$ with trivial nebentypus. In this context, the proportion of nonvanishing for the central value of a single $L$ function was investigated first by Duke in [Du]; subsequently, using mollification techniques, his results were improved by E. Kowalski and the authors in [KM2, V1, KMV1] to yield a large positive proportion of nonvanishing central values.

In their seminal paper [IS] on the Landau-Siegel zero problem, Iwaniec and Sarnak demonstrated the importance of establishing, for a positive proportion of primitive forms, the simultaneous nonvanishing of central values of $L$ functions of

${ }^{\star}$ P.M. is partially supported by NSF Grant DMS-97-2992 and by the Ellentuck fund (by grants to the Institute for Advanced Study) and by the Institut Universitaire de France. 
primitive forms twisted by two different characters. They solved this problem (ineffectively) for the case when the first character is trivial and the second is real. In [KMV2], using mollification of fourth moments, the question of simultaneous nonvanishing for the first character trivial and the second arbitrary was solved. The present work extends the methods of [KMV2] to treat simultaneous nonvanishing for central values of $L$ functions with three twists and discuss applications connected to the Birch-Swinnerton-Dyer conjecture for $J_{0}(q)$.

A primitive form $f \in S_{2}^{*}(q)$ admits a Fourier expansion at infinity

$$
f(z):=\sum_{n \geqslant 1} \sqrt{n} \lambda_{f}(n) e(n z),
$$

with $\lambda_{f}(1)=1$. The Fourier coefficients $\sqrt{n} \lambda_{f}(n)$ are real algebraic integers. For $\chi$ a primitive Dirichlet character of modulus $D$, the twist of $f$ by $\chi$, namely

$$
(f \cdot \chi)(z):=\sum_{n \geqslant 1} \chi(n) \sqrt{n} \lambda_{f}(n) e(n z),
$$

is a cuspidal modular form of level $q D^{2}$ and nebentypus $\chi^{2}$. If $D$ is coprime with $q$ (which we henceforth assume), $f \cdot \chi$ is a primitive form. The associated $L$-function is

$$
L(f \cdot \chi, s)=\sum_{n} \frac{\lambda_{f}(n) \chi(n)}{n^{s}}=\prod_{p}\left(1-\frac{\lambda_{f}(p) \chi(p)}{p^{s}}+\frac{\varepsilon_{q}(p) \chi^{2}(p)}{p^{2 s}}\right)^{-1},
$$

where $\varepsilon_{q}$ is the trivial character modulo $q$. This has analytic continuation to the whole complex plane and satisfies the functional equation [Li]

$$
\Lambda(f \cdot \chi, s)=-\chi(-q) \frac{G(\chi)}{G(\bar{\chi})} \varepsilon_{f} \Lambda(f \cdot \bar{\chi}, 1-s)
$$

where $G(\chi)$ is the Gauss sum, $\varepsilon_{f}= \pm 1$, and

$$
\Lambda(f \cdot \chi, s)=\left(q D^{2}\right)^{s / 2}(2 \pi)^{-s} \Gamma\left(s+\frac{1}{2}\right) L(f \cdot \chi, s) .
$$

When $q$ is squarefree, [Li]

$$
\varepsilon_{f}=\mu(q) \sqrt{q} \lambda_{f}(q) .
$$

Let $\chi_{1}, \chi_{2}, \chi_{3}$ be three distinct primitive characters of moduli $D_{1}, D_{2}, D_{3}$ respectively. In this paper we consider the simultaneous nonvanishing of the central value

$$
L\left(f \cdot \chi_{1}, \frac{1}{2}\right) L\left(f \cdot \chi_{2}, \frac{1}{2}\right) L\left(f \cdot \chi_{3}, \frac{1}{2}\right)
$$

when the level $q$ is prime.

THEOREM 1.1. There exists an effective positive constant c satisfying: for any $\chi_{1}, \chi_{2}, \chi_{3}$ three distinct primitive characters whose conductors $D_{1}, D_{2}, D_{3}$ are squarefree, and such that $\chi_{1}^{2}, \chi_{2}^{2}, \chi_{3}^{2}$ remain primitive with the same moduli, we have

$$
\left|\left\{f \in S_{2}^{*}(q), L\left(f \cdot \chi_{1}, \frac{1}{2}\right) L\left(f \cdot \chi_{2}, \frac{1}{2}\right) L\left(f \cdot \chi_{3}, \frac{1}{2}\right) \neq 0\right\}\right| \geqslant c\left|S_{2}^{*}(q)\right|
$$

for all sufficiently large primes $q$, where the requisite size of $q$ depends only on $D_{1}, D_{2}, D_{3}$. 
As is now standard, this is proved through mollification techniques, combined with estimates for the twisted fourth moments of $L\left(f \cdot \chi_{i}, 1 / 2\right)$. Bounds on moments of this sort are analyzed thoroughly in [DFI2], and indeed we adopt their methods to control the largest remainder terms. However, for mollification we also need the asymptotics of these moments. When $\chi_{i}$ is the trivial character these asymptotics were computed for the first time in [KMV2], and the bulk of the present paper is spent obtaining the necessary variant when $\chi_{i}$ is nontrivial. There is no significant distinction in the handling of the remainder terms in this case, but the asymptotics are considerably more intricate (since the Eisenstein series arising from $\chi \otimes \bar{\chi}$ has square level). The two assumptions on $\chi$ in Theorem 1.1 are there to simplify the calculations, not because we expect the results to be false in other settings. For the applications we have in mind (see below) the assumptions do not cause us any significant difficulties.

In the course of the proof, we obtain the following precise asymptotic for the fourth moment:

PROPOSITION 1.2. Let $\chi$ be a complex primitive character of squarefree conductor $D>1$, such that $\chi^{2}$ is primitive. If $q$ is prime, then

$$
\begin{aligned}
& \sum_{f \in S_{2}^{*}(q)} \frac{1}{4 \pi(f, f)}|L(f \cdot \chi, 1 / 2)|^{4} \\
& \quad=P_{\chi}\left(\log \left(q D^{2} / 4 \pi^{2}\right)\right)+2 \Re e\left(\chi(q) C_{\chi}\right)+O_{\varepsilon, D}\left(q^{-1 / 12+\varepsilon}\right),
\end{aligned}
$$

where $C_{\chi}$ is a constant depending on $\chi$ only and $P_{\chi}(X)$ is a polynomial of degree 4 , whose coefficients depend only on $\chi$, with leading coefficient

$$
\frac{1}{12 \zeta(2)} \prod_{p \mid D} \frac{(1-1 / p)^{3}}{(1+1 / p)}\left|L\left(\chi^{2}, 1\right)\right|^{2} .
$$

This should be compared with Corollary 1.3 of [KMV2] which treated the case of the trivial character and where the degree of the corresponding polynomial is 6 . The difference in degrees is explained by the fact that the family $\{L(f \cdot \chi, s)\}_{f \in S_{2}^{*}(q)}$ is predicted to admit an 'unitary' symmetry (in the terminology of [KS]) when $\chi$ is complex, and an 'orthogonal' symmetry when $\chi$ is trivial and $f$ is restricted to 'even forms'. In both cases our computations fit well with the predictions of Conrey and Farmer $[\mathrm{CF}]$ and Keating and Snaith [KeSn] using random matrix models. Note that $C_{\chi}$ and the coefficients of $P_{\chi}$ can be explicitly computed.

\subsection{ARITHMETIC APPLICATIONS}

These investigations on simultaneous nonvanishing were motivated by the recent progress made towards the Birch-Swinnerton-Dyer conjecture for quotients of $J_{0}(q)$ (the Jacobian of the modular curve $X_{0}(q)$ ) over abelian number fields. Let $K$ be an Abelian extension of $\mathbf{Q}$. By the Kronecker-Weber Theorem, $K$ is a subfield of some cyclotomic extension $\mathbf{Q}(\exp (2 \pi \mathrm{i} / D))$. Identifying $\operatorname{Gal}(\mathbf{Q}(\exp (2 \pi \mathrm{i} / D)))$ with 
$(\boldsymbol{Z} / D \boldsymbol{Z})^{*}$, there exists a subgroup $\widehat{\mathrm{Gal}(K)}$ of the Dirichlet characters of modulus $D$ (which is identified with the group of characters of $\operatorname{Gal}(K)$ ) such that the Dedekind $L$-function of the field $K$ takes the form

$$
L(K, s):=\prod_{\mathfrak{p} \in \operatorname{Spec}\left(O_{K}\right)}\left(1-\frac{1}{N_{K / \mathbf{Q}}(\mathfrak{p})^{s}}\right)^{-1}=\prod_{\chi \in \operatorname{Gal}(K)} L(\tilde{\chi}, s),
$$

where $\tilde{\chi}$ is the underlying primitive character of $\chi$. For $f \in S_{2}^{*}(q)$, by successive applications of the cyclic base change theory (due to Saito, Shintani and Langlands [La]) there is an automorphic form $f_{K}$ over $G L_{2}\left(\mathbf{A}_{K}\right)$ called the base change lift of $f$ from $\mathbf{Q}$ to $K$ whose associated $L$ function is given by

$$
L\left(f_{K}, s\right)=\prod_{\chi \in \operatorname{Gal}(K)} L(f \cdot \tilde{\chi}, s) .
$$

Hence, Theorem 1.1 implies the following

COROLLARY 1.3. There exists an effective positive constant $c$ satisfying: let $K$ be a Galois extension of degree 5 unramified at 5 , then for any sufficiently large prime $q$ (depending on $K)\left|\left\{f \in S_{2}^{*}(q), L\left(f_{K}, \frac{1}{2}\right) \neq 0\right\}\right| \geqslant c\left|S_{2}^{*}(q)\right|$.

The condition that 5 is unramified insures that the conductor of any $\chi \in \widehat{\operatorname{Gal}(K)}$ is square-free. The corollary fits with the following results proven in [KMV2]:

THEOREM. There exists an effective positive constant c satisfying: let $K$ be a Galois extension of degree 2 or 3 , then for any sufficiently large prime $q$ (depending on $K$ )

$$
\left|\left\{f \in S_{2}^{*}(q), L\left(f_{K}, \frac{1}{2}\right) \neq 0\right\}\right| \geqslant c\left|S_{2}^{*}(q)\right|,
$$

if $K$ is quadratic and $\chi_{K}(-q)=1$ (here $\chi_{K}$ is the Kronecker symbol of $K$ ) or if $K$ is cubic. If $K$ is quadratic and $\chi_{K}(-q)=-1$ then

$$
\mid\left\{f \in S_{2}^{*}(q), \text { ord }_{s=\frac{1}{2}} L\left(f_{K}, s\right)=1\right\}|\geqslant c| S_{2}^{*}(q) \mid .
$$

Note that we have said nothing so far about extensions of degree 4 . The following variant of Theorem 1.1, along with its corollary, takes care of the case of cyclic extensions:

THEOREM 1.4. There exists an effective positive constant $c$ satisfying: let $\chi_{2}$ a real character and $\chi_{3}$ a complex character of squarefree conductors $D_{2}, D_{3}$ such that $\chi_{D_{3}}^{2}$ is primitive, then

$$
\begin{aligned}
& \mid\left\{f \in S_{2}^{*}(q), \chi_{2}(-q)=1, \text { ord }_{s=\frac{1}{2}} L(f, s) L\left(f \cdot \chi_{2}, s\right) L\left(f \cdot \chi_{3}, s\right)=0\right\}|\geqslant c| S_{2}^{*}(q) \mid, \\
& \mid\left\{f \in S_{2}^{*}(q), \chi_{2}(-q)=-1, \text { ord }_{s=\frac{1}{2}} L(f, s) L\left(f \cdot \chi_{2}, s\right) L\left(f \cdot \chi_{3}, s\right)=1\right\}|\geqslant c| S_{2}^{*}(q) \mid,
\end{aligned}
$$

for all sufficiently large primes $q$ (depending on $D_{2}, D_{3}$ ). 
COROLLARY 1.5. There exists an effective positive constant $c$ satisfying: let $K$ be a cyclic extension of degree 4 unramified at 2 and such that $K$ is unramified over its (unique) quadratic subfield $K^{\prime}$. Let $\chi_{K^{\prime}}$ be the Kronecker symbol associated to $K^{\prime}$, then for any $q$ prime large enough (depending on $K$ )

$$
\begin{aligned}
& \left|\left\{f \in S_{2}^{*}(q), \chi_{K^{\prime}}(-q)=1, \operatorname{ord}_{s=\frac{1}{2}} L\left(f_{K}, s\right)=0\right\}\right| \geqslant c\left|S_{2}^{*}(q)\right| . \\
& \left|\left\{f \in S_{2}^{*}(q), \chi_{K^{\prime}}(-q)=-1, \operatorname{ord}_{s=\frac{1}{2}} L\left(f_{K}, s\right)=1\right\}\right| \geqslant c\left|S_{2}^{*}(q)\right| .
\end{aligned}
$$

We will not give the proof of Theorem 1.4 here as it turns out to be easier in many aspects than that of Theorem 1.1; however we shortly discuss how this variant is obtained in Section 11.

Remark. The remaining degree four case is that of the biquadratic field. By our methods this would require precise asymptotics for the twisted sixth moments of $L(f \cdot \chi, 1 / 2)$, which are beyond the reach of current techniques.

Combining these results with the work of Gross, Zagier, Kolyvagin, Logachev, and Kato, [GZ, Ko, KL, Ru, Sc] we obtain the following theorem:

THEOREM 1.6. Let $K$ be a cyclic extention of $\mathbf{Q}$ of degree at most five such that 2 (respectively 5 ) is unramified if $[K: \mathbf{Q}]=4$ (respectively 5 ). If $K$ is quartic assume also that $K$ is unramified over its unique quadratic subfield. There exists an absolute positive constant $c$ such that for $q$ a sufficiently large prime (depending on $K$ ), $J_{0}(q)$ admits a quotient $J$ of dimension $\operatorname{dim} J \geqslant c \operatorname{dim} J_{0}(q)$, whose group of $K$-rational points satisfies the weak Birch-Swinnerton-Dyer conjecture. More precisely, denote by $J_{K}$ the $K$ rational abelian variety obtained from $J$ by extension of scalars to $K$, and by $L\left(J_{K}, s\right)$ the associated L-function. Then

$$
\begin{aligned}
\operatorname{rank}_{\mathrm{Z}} J(K) & =\operatorname{ord}_{s=1} L\left(J_{K}, s\right) \\
& =\left\{\begin{array}{cl}
\operatorname{dim} J & \text { if } K \text { contains } K^{\prime}, \text { quadratic, with } \chi_{K^{\prime}}(-q)=-1, \\
0 & \text { else. }
\end{array}\right.
\end{aligned}
$$

Our paper is organized as follows: after introducing some notation and definitions in Section 2, we show in Section 3 how Theorem 1.1 and its variant 1.4 implies the Corollaries 1.3, 1.5, and 1.6. In Section 4 we explain how the proof of Theorem 1.1 reduces to the estimate of (mollified) third and fourth moments. The triple moment is computed in Sections 5 and 6. The computation of the fourth moment is handled in Sections 7, 8 and 9. In Section 7, we isolate the main terms and compute the easiest ones, the so-called 'diagonal' and 'off-diagonal' terms. In Sections 8 and 9 we compute the third main term (which, lacking a better term, we call 'off-off-diagonal'); this is by far the most difficult and technical portion of the paper. The key is that, as in [KMV2], the off-off-diagonal term is defined by a double integral whose integrand is an odd function of both variables ( $s, t$ say); hence by contour shift, the OOD term equals the residue of the integrand at $s=t=0$ (see (9.5) and (9.10)). Let us 
emphasize that without this rather delicate property, it is not possible to mollify properly the OOD portion. The mollification (and the completion of the proof of Theorem 1.1) is sketched in Section 10, most of the techniques being those of [KMV2] Sections 5 to 7. In Section 11 we discuss the proof of Theorem 1.4. We end with a series of appendices dealing with some general forms of the Poisson formula and the study of various exponential sums encountered in this paper.

\section{Notation and Formulae}

We refer the reader to [KMV2] for background on modular forms. In addition to the formulae mentioned above, we will also use the multiplicative relation for the coefficients of Hecke eigenforms in $S_{2}^{*}(q)$ :

$$
\lambda_{f}(m) \lambda_{f}(n)=\sum_{d \mid(m, n)} \varepsilon_{q}(d) \lambda_{f}\left(\frac{m n}{d^{2}}\right) .
$$

Mobius inversion then gives the inverse equation

$$
\lambda_{f}(m n)=\sum_{d \mid(m, n)} \varepsilon_{q}(d) \mu(d) \lambda_{f}\left(\frac{m}{d}\right) \lambda_{f}\left(\frac{n}{d}\right),
$$

where $\varepsilon_{q}$ is the trivial character modulo $q$.

The fundamental formula of this paper is Petersson's trace formula, which we now review. Let $\mathcal{B}_{2}(q)$ be an orthogonal basis of $S_{2}^{*}(q)$. Then

$$
\sum_{f \in \mathcal{B}_{2}(q)} \frac{\psi_{f}(m) \bar{\psi}_{f}(n)}{4 \pi(f, f)}=\delta_{m, n}+\Delta(m, n)
$$

with

$$
\Delta(m, n):=-2 \pi \sum_{\substack{c \equiv 0(q) \\ c>0}} \frac{S(m, n ; c)}{c} J_{1}\left(\frac{4 \pi \sqrt{m n}}{c}\right)
$$

and

$$
S(m, n ; c)=\sum_{x(c),(x, c)=1} e\left(\frac{m x+n \bar{x}}{c}\right) .
$$

Note that the identity (2.3) is independent of the choice of the basis $\mathcal{B}_{2}(q)$; since in this paper $q$ is prime, there are no 'oldforms' and $S_{2}^{*}(q)$ is an orthogonal basis of $S_{2}^{*}(q)$. In particular we have

$$
\left|S_{2}^{*}(q)\right|=\operatorname{dim} S_{2}(q)=\frac{q}{12}+\mathrm{O}(1)
$$

To simplify later discussions, we introduce some notation. Given an Euler product $L(s)=\prod_{p} L_{p}(s)$ and an integer $A$, let $L_{A}(s)=\prod_{p \mid A} L_{p}(s)$ and $L^{(A)}(s)=$ $\prod_{(p, A)=1} L_{p}(s)$. To avoid confusion, the $A$ th power of $L(s)$ will be noted $L(s)^{A}$. Given an integer $h$ and a character $\chi$ of modulus $D$, we let $G(\chi, h)=\sum_{x(D)} \chi(x) e(h x / D)$ denote the Gauss sum and let $G(\chi)$ denote $G(\chi, 1)$. When the modulus of $\chi$ is not clear 
from the context (for example if $\chi$ is induced from a character of lower level) we use the more precise notation $G(\chi, h ; D)$. We let $\tilde{\chi}$ denote the unique primitive character inducing $\chi$. If $D=\prod_{p} p^{\alpha_{p}}$, we factor $\chi()$ into $\chi(*):=\prod_{p \mid D} \chi_{p}(*)$ where $\chi_{p}$ are characters of modulus $p^{\alpha_{p}}$.

Because (2.3) will be used for essentially all averaging over forms in this paper, it will be convenient to introduce the the following notation for the weighted average of forms:

$$
\sum_{f \in S_{2}^{*}(q)}^{h} \alpha_{f}:=\sum_{f \in S_{2}^{*}(q)} \frac{\alpha_{f}}{4 \pi(f, f)} .
$$

\section{Derivation of the Arithmetic Applications}

In this section we show how Theorem 1.1 and its variant, Theorem 1.4, imply 1.3, 1.5 and Theorem 1.6. For $K$ a cyclic extension of degree at most five we let $\operatorname{Gal}(K)$ denote its Galois group over $\mathbf{Q}$ and $\widehat{\operatorname{Gal}(K)}$ its character group. Let $\chi$ be the generator of $\widehat{\operatorname{Gal}(K)}$. Then for $f \in S_{2}^{*}(q)$,

$$
L\left(f, \frac{1}{2}\right) L\left(f \cdot \chi, \frac{1}{2}\right) L\left(f \cdot \chi^{2}, \frac{1}{2}\right) \neq 0 \quad \Longleftrightarrow \quad L\left(f, \frac{1}{2}\right) L\left(f \cdot \bar{\chi}, \frac{1}{2}\right) L\left(f \cdot \bar{\chi}^{2}, \frac{1}{2}\right) \neq 0
$$

and, since $1, \chi, \chi^{2}, \bar{\chi}$, and $\bar{\chi}^{2}$ cover all of $\widehat{\operatorname{Gal}(K)}$, we have

$$
L\left(f_{K}, \frac{1}{2}\right)=\prod_{\chi \in \operatorname{Gal}(K)} L\left(f \cdot \chi, \frac{1}{2}\right) \neq 0 .
$$

Thus, Theorem 1.1 implies Corollary 1.3 and similarly Theorem 1.4 implies Corollary 1.5 .

Our starting point in the derivation of Theorem 1.6 is the work of Shimura [SH] on the arithmeticity of central values of $L$ functions: for any $\sigma \in \operatorname{Gal}(\overline{\mathrm{Q}}), f \in S_{2}^{*}(q)$, and character $\chi$,

$$
L(f \cdot \chi, 1 / 2) \neq 0 \quad \Longleftrightarrow \quad L\left(f^{\sigma} \cdot \chi^{\sigma}, 1 / 2\right) \neq 0 .
$$

When $\chi$ is real, it follows from [GZ] that

$$
\operatorname{ord}_{s=\frac{1}{2}} L(f \cdot \chi, s)=1 \Longleftrightarrow \operatorname{ord}_{s=\frac{1}{2}} L\left(f^{\sigma} \cdot \chi^{\sigma}, s\right)=1
$$

(here by $L(f \cdot \chi, s)$ we really mean $L(f \cdot \tilde{\chi}, s)$, the $L$-function of the twist by the primitive underlying character). From this it follows that

$$
\prod_{\chi \in \operatorname{Gal}(K)} L(f \cdot \chi, 1 / 2) \neq 0 \Leftrightarrow \prod_{\chi \in \operatorname{Gal}(K)} L\left(f^{\sigma} \cdot \chi^{\sigma}, 1 / 2\right) \neq 0 \Leftrightarrow \prod_{\chi \in \operatorname{Gal}(K)} L\left(f^{\sigma} \cdot \chi, 1 / 2\right) \neq 0,
$$

since $\sigma$ stabilizes $\widehat{\operatorname{Gal}(K)}$. More precisely, if $\chi$ has order $d$ and $\sigma(\exp (2 \pi \mathrm{i} / d))=$ $\exp (2 \pi \mathrm{i} a / d)$ for $(a, d)=1$ then $\chi^{\sigma}=\chi^{a}$. It follows that

$$
\prod_{\chi \in \operatorname{Gal}(K)} L(f \cdot \chi, 1 / 2) \neq 0 \Longleftrightarrow \forall \chi \in \widehat{\operatorname{Gal}(K)}, \prod_{f^{\sigma},\{\sigma \in \operatorname{Gal}(\bar{Q})\}} L\left(f^{\sigma} \cdot \chi, 1 / 2\right) \neq 0 .
$$


Let $A_{f}$ denote the subquotient of $J_{0}(q)$ associated to $f$ by Shimura theory. This is an Abelian variety of dimension $2 d_{f}$. Let $A_{f}^{K}=\operatorname{Res}_{K / \mathbf{Q}} A_{f / K}$ be the restriction from $K$ to $\mathbf{Q}$ of the extension of scalars of $A_{f}$ to $K$; this is an Abelian variety over $\mathbf{Q}$ of dimension $(\operatorname{deg} K)\left(\operatorname{dim} A_{f}\right)$. The set of rational points $A_{f}^{K}(\mathbf{Q})$ is naturally isomorphic as a $\mathrm{Z}$-module to the set of $K$-rational points of $A_{f}$; in particular there is an action of $\operatorname{Gal}(K)$ on $A_{f}^{K}(\mathbf{Q})$ and a natural decomposition of $A_{f}^{K}(\mathbf{Q}) \otimes \mathbf{C}$ into $\chi$ eigencomponents for $\chi \in \operatorname{Gal}(K)$,

$$
A_{f}^{K}(\mathbf{Q}) \otimes \mathbf{C}=\bigoplus_{\chi \in \operatorname{Gal}(K)}\left(A_{f}^{K}(\mathbf{Q}) \otimes \mathbf{C}\right)^{\chi}
$$

By the work of $\mathrm{Kato}^{\star}$ (see $[\mathrm{Ru}, \mathrm{Sc}]$ and the discussion in Section 5 of [Me]),

$$
\prod_{f^{\prime} \in\left\{f^{\sigma}, \sigma \in \operatorname{Gal}(\bar{Q})\right\}} L\left(f^{\sigma} \cdot \chi, 1 / 2\right) \neq 0 \text { implies that } \operatorname{dim}\left(A_{f}^{K}(\mathbf{Q}) \otimes \mathbf{C}\right)^{\chi}=0 .
$$

hence

$$
L\left(f_{K}, \frac{1}{2}\right) \neq 0 \text { implies that } \operatorname{rank} A_{f}(K)=0 .
$$

If $K$ contains a quadratic field $K^{\prime}$ such that $\chi(-q)=-1$, then

$$
L\left(f, \frac{1}{2}\right) L\left(f \cdot \chi_{K^{\prime}}, \frac{1}{2}\right)=L\left(f_{K}, \frac{1}{2}\right)=0,
$$

so if we assume that $\operatorname{ord}_{\frac{1}{2}} L\left(f_{K}, s\right)=1$ when either

$$
L\left(f, \frac{1}{2}\right) \neq 0 \quad \text { or } \quad L\left(f \cdot \chi_{K^{\prime}}, \frac{1}{2}\right) \neq 0,
$$

and that $L\left(f \cdot \chi, \frac{1}{2}\right) \neq 0$ for all the other $\left.\chi \in \widehat{\operatorname{Gal}(K}\right)$, then this argument continues to hold and for all $\chi \in \widehat{\operatorname{Gal}(K)}$ with one exception the corresponding $\chi$-eigencomponent is zero-dimensional. Suppose that the exception is the trivial character: ord $_{s=\frac{1}{2}}$ $L(f, s)=1$; then from the $[\mathrm{GZ}, \mathrm{Ko}, \mathrm{KL}]$,

$$
\operatorname{rank} A_{f}(\mathbf{Q})=\operatorname{ord}_{s=\frac{1}{2}} L\left(A_{f}, s\right)=d_{f},
$$

hence in this case

$$
\operatorname{ord}_{s=\frac{1}{2}} L\left(f_{K}, s\right)=1 \text { implies that } \operatorname{rank} A_{f}(K)=d_{f} \text {. }
$$

The same argument holds in the case ord ${ }_{s=\frac{1}{2}} L\left(f \cdot \chi_{K^{\prime}}, s\right)=1$ with a minor difficulty: one needs instead to consider the product $A_{f} \times A_{f \cdot \chi_{K^{\prime}}}$ where $A_{f \cdot \chi_{K^{\prime}}}$ is the subquotient of $J_{0}\left(q\left(\operatorname{cond} \chi_{K^{\prime}}\right)^{2}\right)$ corresponding to the twisted form $f \cdot \chi_{K^{\prime}} \in S_{2}^{*}\left(q\left(\operatorname{cond} \chi_{K^{\prime}}\right)^{2}\right)$. From the hypotheses $\operatorname{ord}_{\frac{1}{2}} L\left(f \cdot \chi_{K^{\prime}}, s\right)=1, L\left(f, \frac{1}{2}\right) \neq 0$ we have $\operatorname{rank} A_{f} \times A_{f \cdot \chi_{K^{\prime}}}=d_{f \cdot \chi_{K^{\prime}}}=d_{f}$.

To conclude the derivation of Theorem 1.6, we consider, for $K$ cyclic of degree at most five, the quotient $J=\prod_{f} A_{f}$, where $f$ ranges through a set of representatives of $\operatorname{Gal}(\overline{\mathrm{Q}})$-orbits of $f \in S_{2}^{*}(q)$ such that the order of $L\left(f_{K}, s\right)$ at $s=\frac{1}{2}$ is 1 or 0 , depending on whether $K$ does or does not contain a quadratic subfield $K^{\prime}$ such that $\chi_{K^{\prime}}(-q)=-1$. From (3.2) and (3.3) $J(K)$ has the expected rank. The condition that

\footnotetext{
${ }^{\star}$ If $\chi$ is real, one uses the work of Gross and Zagier and Kolyvagin and Logachev instead.
} 
$K$ is unramified at 2 or 5 if $\operatorname{deg} K=4$ or 5 , respectively, insures that the conductors of the characters of $\operatorname{Gal}(K)$ are coprime with their order and hence are squarefree, so, from Corollaries 1.3 and 1.5 we have for $q$ a sufficiently large prime

$$
\operatorname{dim} J \geqslant 2 c\left|S_{2}^{*}(q)\right|=2 c \operatorname{dim} J_{0}(q) .
$$

\section{Principle of the Proof of Nonvanishing}

The basic idea of the proof is that for any numbers $L_{1}(f), L_{2}(f), L_{3}(f)$ one has by Hölder's inequality

$$
\begin{aligned}
& \left(\sum_{f \in S_{2}^{*}(q)} L_{1}(f) L_{2}(f) L_{3}(f)\right)^{4} \\
& \quad \leqslant\left(\sum_{\substack{f \in \epsilon_{2}^{*}(q) \\
L_{1} L_{2} L_{3}(f) \neq 0}} 1\right)\left(\sum_{f \in S_{2}^{*}(q)}\left|L_{1}(f)\right|^{4}\right)\left(\sum_{f \in S_{2}^{*}(q)}\left|L_{2}(f)\right|^{4}\right)\left(\sum_{f \in S_{2}^{*}(q)}\left|L_{3}(f)\right|^{4}\right) .
\end{aligned}
$$

We take $L_{i}(f)=M_{i}(f) L\left(f \cdot \chi_{i}, \frac{1}{2}\right)$ where $M_{i}(f)$ is a 'mollifier' chosen so that

$$
\sum_{f \in S_{2}^{*}(q)} \frac{L_{1}(f) L_{2}(f) L_{3}(f)}{\left|S_{2}^{*}(q)\right|} \geqslant c_{0}+o(1) \quad \text { and } \quad \sum_{f \in S_{2}^{*}(q)} \frac{\left|L_{i}(f)\right|^{4}}{\left|S_{2}^{*}(q)\right|} \leqslant c_{i}+\mathrm{o}(1) \text {, }
$$

where $c_{0}, c_{1}, c_{2}, c_{3}$ are positive. In the following sections we will show that

$$
\left|\sum_{f \in S_{2}^{*}(q)}^{h} L_{1}(f) L_{2}(f) L_{3}(f)\right| \geqslant\left(c_{0}+\mathrm{o}(1)\right)(\log q)^{k_{0}}
$$

and

$$
\sum_{f \in S_{2}^{*}(q)}\left|L_{i}(f)\right|^{4} \leqslant\left(c_{i}+\mathrm{o}(1)\right)(\log q)^{k_{i}},
$$

for $i=1,2,3$, with $4 k_{0}=k_{1}+k_{2}+k_{3}$ so that

$$
\frac{c_{0}^{4}}{c_{1} c_{2} c_{3}}+\mathrm{o}(1) \leqslant \sum_{\substack{s_{\in} S_{2}^{*}(q) \\ L_{1} L_{2} L_{3}(f) \neq 0}}^{h} 1
$$

To finish the proof of Theorem 1.1, we must still replace the weight $1 / 4 \pi(f, f)$ by the 'natural' weight $1 /\left|S_{2}^{*}(q)\right|$. This can be done using the axiomatic system of [KM2] and [KMV2] with no loss in the size of the constants, but since we do not care about the size of the constants, it is enough to use the inequality (already used by Luo in [Lu2], in a similar context)

$$
\sum_{\substack{f \in \sum_{2}^{*}(q) \\ L_{1} L_{2} L_{3}(f) \neq 0}}^{h} 1 \leqslant\left(\sum_{\substack{f \in S_{2}^{*}(q) \\ L_{1} L_{2} L_{3}(f) \neq 0}} 1\right)^{1 / 2}\left(\sum_{f \in S_{2}^{*}(q)}^{h} \frac{1}{4 \pi(f, f)}\right)^{1 / 2}
$$

and the upper bound due to E. Royer [Ro]: 
LEMMA 4.1. There exists an absolute constant $C$ such that, for $q$ a sufficiently large prime,

$$
\sum_{f \in S_{2}^{*}(q)}^{h} \frac{1}{4 \pi(f, f)} \leqslant C q^{-1} .
$$

\section{The Third Moment}

\subsection{EXPRESSION OF THE CENTRAL VALUES AS RAPIDILY CONVERGING SERIES}

Let $\chi$ be a primitive character of conductor $D$, and let $\varepsilon_{\chi}=-\chi(-q) G(\chi) / G(\bar{\chi})$. Take $f \in S_{2}^{*}(q)$ with $(q, D)=1$. Then from (1.1) and a contour shift we have

where

$$
L\left(f \cdot \chi, \frac{1}{2}\right)=\sum_{n} \frac{\lambda_{f}(n) \chi(n)}{n^{1 / 2}} V\left(\frac{n}{q^{1 / 2} D}\right)+\varepsilon_{\chi} \varepsilon_{f} \sum_{n} \frac{\lambda_{f}(n) \chi(n)}{n^{1 / 2}} V\left(\frac{n}{q^{1 / 2} D}\right)
$$

$$
V(y)=\frac{1}{2 \pi \mathrm{i}} \int_{(3)} \Gamma(1+s)(2 \pi y)^{-s} \frac{\mathrm{d} s}{s} .
$$

If $\chi$ is real then

$$
\left(\frac{\sqrt{q} D}{2 \pi}\right)^{-1} \Lambda^{\prime}\left(f \cdot \chi, \frac{1}{2}\right)=\left(1-\varepsilon_{\chi} \varepsilon_{f}\right) \sum_{n} \frac{\lambda_{f}(n)}{n^{1 / 2}} V\left(\frac{n}{q^{1 / 2} D}\right) .
$$

For $\chi_{1}, \chi_{2}$ two primitive characters of respective moduli $D_{1}, D_{2}$, and $\left(q, D_{1} D_{2}\right)=1$, we denote by

$$
\chi_{1} * \chi_{2}(n)=\sum_{n_{1} n_{2}=n} \chi_{1}\left(n_{1}\right) \chi_{2}\left(n_{2}\right)
$$

their Dirichlet convolution. By (2.1), we get

$$
L\left(f \cdot \chi_{1}, s\right) L\left(f \cdot \chi_{2}, s\right)=L^{(q)}\left(2 s, \chi_{1} \chi_{2}\right) \sum_{n} \lambda_{f}(n) \chi_{1} * \chi_{2}(n) n^{-s}
$$

so that

$$
\begin{aligned}
L\left(f \cdot \chi_{1}, \frac{1}{2}\right) L\left(f \cdot \chi_{2}, \frac{1}{2}\right)= & \sum_{n} \frac{\lambda_{f}(n) \chi_{1} * \chi_{2}(n)}{n^{1 / 2}} W_{\chi_{1} \chi_{2}}\left(\frac{n}{q D_{1} D_{2}}\right)+ \\
& +\varepsilon_{\chi_{1}} \varepsilon_{\chi_{2}} \sum_{n} \frac{\lambda_{f}(n) \bar{\chi}_{1} * \bar{\chi}_{2}(n)}{n^{1 / 2}} W_{\overline{\chi_{1} \chi_{2}}}\left(\frac{n}{q D_{1} D_{2}}\right) \\
:= & P\left(f, \chi_{1} \chi_{2}\right)+\varepsilon_{\chi_{1}} \varepsilon_{\chi_{2}} P\left(f, \bar{\chi}_{1} \bar{\chi}_{2}\right)
\end{aligned}
$$

say, with

$$
W_{\chi_{1} \chi_{2}}(y)=\frac{1}{2 \pi \mathrm{i}} \int_{(3)} \Gamma(1+s)^{2} L^{(q)}\left(1+2 s, \chi_{1} \chi_{2}\right)\left(4 \pi^{2} y\right)^{-s} \frac{\mathrm{d} s}{s} .
$$

Shifting the line of integration, we infer that

$$
y^{j} V^{(j)}(y), y^{j} W_{\chi_{1} \chi_{2}}^{(j)}(y) \ll(1+y)^{-A}|\log y|
$$


for all $j \geqslant 0$ and all $A>0$, the implied constant depending on $j, A, D_{1}, D_{2}$ only (the $|\log y|$ factor appearing if and only if $\chi_{1}=\bar{\chi}_{2}$ ). On the other hand, if we shift the line to $\Re e s=-1 / 2$ we have (if $\chi_{1} \neq \bar{\chi}_{2}$ )

$$
W_{\chi_{1} \chi_{2}}(y)=L\left(\chi_{1} \chi_{2}, 1\right)+O_{D_{1}, D_{2}}\left(y^{1 / 2}\right) .
$$

\subsection{MOLLIFICATION}

Let $\chi_{1}, \chi_{2}, \chi_{3}$ be distinct primitive Dirichlet characters of conductors $D_{1}, D_{2}, D_{3}$, and let $q$ be a large prime with $\left(q, D_{1} D_{2} D_{3}\right)=1$. Since $L\left(f \cdot \bar{\chi}, \frac{1}{2}\right)=\overline{L\left(f \cdot \chi, \frac{1}{2}\right)}$ we may also assume that $\chi_{i} \neq \bar{\chi}_{j}$ for $i \neq j$. For each $f \in S_{2}^{*}(q)$ and $\chi_{i}, i=1,2,3$ we associate a mollifier $M_{i}(f)$ of the form

$$
M_{i}(f)=\sum_{\substack{\ell \leqslant L \\(\ell, q)=1}} x_{i}(\ell) \frac{\lambda_{f}(\ell)}{\ell^{1 / 2}},
$$

where $L:=q^{\Delta}$ for some fixed $\Delta<1$ and the $x_{i}(\ell)$ are complex numbers satisfying $x_{i}(\ell) \ll q^{\varepsilon}$ for all $\varepsilon>0$, the implied constant depending on $\varepsilon, D_{1}, D_{2}, D_{3}$ only. To simplify the notations to come we note that (2.1) lets us write

$$
M_{1}(f) M_{2}(f) M_{3}(f)=: M(f)=\sum_{\substack{\ell \leqslant L^{\prime} \\(\ell, q)=1}} \frac{x(\ell)}{\ell^{1 / 2}},
$$

with $L^{\prime}=q^{3 \Delta}$ and the $x(\ell)$ satisfying the same bound. To be precise, we have

$$
x(\ell)=\sum_{\mathrm{d}, \mathrm{d}^{\prime}} \frac{1}{\mathrm{dd}^{\prime}} \sum_{\ell_{3} \ell_{4}=\ell} \sum_{\ell_{1} \ell_{2}=\ell_{3} \mathrm{~d}^{\prime}} x_{1}\left(\mathrm{~d} \ell_{1}\right) x_{2}\left(\mathrm{~d} \ell_{2}\right) x_{3}\left(\mathrm{~d}^{\prime} \ell_{3}\right) .
$$

In view of (5.1) and (5.3), it suffices to estimate the sums

and

$$
P M\left(\chi_{1}, \chi_{2}, \chi_{3}\right):=\sum_{f}^{h} P\left(f, \chi_{1} \chi_{2}\right) P\left(f, \chi_{3}\right) M(f)
$$

$$
P M^{\varepsilon}\left(\chi_{1}, \chi_{2}, \bar{\chi}_{3}\right):=\sum_{f}^{h} \varepsilon_{f} P\left(f, \chi_{1} \chi_{2}\right) P\left(f, \bar{\chi}_{3}\right) M(f)
$$

and to take an appropriate linear combination involving the conjugates of $\chi_{1}, \chi_{2}$. We make an additionial reduction by writing $P\left(f, \chi_{3}\right) M(f)$ in the form

$$
\begin{aligned}
P\left(f, \chi_{3}\right) M(f) & =\sum_{\ell, n} \frac{\lambda_{f}(\ell n)}{(\ell n)^{1 / 2}} \sum_{(d, q)=1} \frac{x(\mathrm{~d} \ell) \chi_{3}(\mathrm{~d} n)}{\mathrm{d}} V\left(\frac{\mathrm{d} n}{q^{1 / 2} D_{3}}\right) \\
& :=\sum_{n} \frac{\lambda_{f}(n)}{n^{1 / 2}} y(n) .
\end{aligned}
$$

Note that, in view of (5.4) and the bound on $x(\ell)$,

$$
y(n) \ll q^{\varepsilon}\left(1+\frac{n}{q^{1 / 2+3 \Delta}}\right)^{-A}
$$

for all $\varepsilon, A>0$ the implied constant depending on $\varepsilon, A$, and $D_{1} D_{2} D_{3}$. 


\subsection{EVALUATION OF $P M\left(\chi_{1}, \chi_{2}, \chi_{3}\right)$}

Since $q$ is prime, we have $\mathcal{B}_{2}(q)=S_{2}^{*}(q)$ and Petersson's formula provides a decomposition of $P M\left(\chi_{1}, \chi_{2}, \chi_{3}\right)$ into the sum of a diagonal term,

$$
\sum_{m} \frac{y(m) \chi_{1} * \chi_{2}(m)}{m} W_{\chi_{1} \chi_{2}}\left(\frac{m}{q D_{1} D_{2}}\right)
$$

and a nondiagonal term,

$$
\begin{aligned}
P M^{N D}= & -2 \pi \sum_{c>0, c \equiv 0(q)} c^{-1} \sum_{m, n} \frac{\chi_{1} * \chi_{2}(m)}{m^{1 / 2}} W_{\chi_{1} \chi_{2}}\left(\frac{m}{q D_{1} D_{2}}\right) \frac{y(n)}{n^{1 / 2}} \times \\
& \times S(m, n ; c) J_{1}\left(\frac{4 \pi \sqrt{m n}}{c}\right) .
\end{aligned}
$$

Now we appeal to the large sieve inequality of [DFI2]:

PROPOSITION 5.1. Let $k \geqslant 2$ be an integer. For $\eta$ a smooth function supported in $[C, 2 C]$ such that $\eta^{(i)} \ll_{i} C^{-i}$ for all $i \geqslant 0$, set

$$
\Delta_{\eta}(n, \ell):=2 \pi i^{-k} \sum_{\substack{c \equiv 0(q) \\ c>0}} \frac{S(n, \ell ; c)}{c} J_{k-1}\left(\frac{4 \pi \sqrt{\ell n}}{c}\right) \eta(c) .
$$

Then for any sequences of complex numbers $x_{m}, y_{n}$,

$$
\sum_{m \leqslant M} \sum_{n \leqslant N} x_{m} y_{n} \Delta_{\eta}(\ell, n) \ll_{\varepsilon, k} C^{\varepsilon}\left(\frac{\sqrt{L N}}{C}\right)^{k-3 / 2}\left(1+\frac{L}{q}\right)^{1 / 2}\left(1+\frac{N}{q}\right)^{1 / 2}\|\mathbf{x}\|_{2}\|\mathbf{y}\|_{2}
$$

with any $\varepsilon>0$ the implied constant depending on $\varepsilon$ and $k$ only.

Introducing a smooth partition of unity on the variables $c, m, n$ and using Proposition 5.1 along with (5.4) and (5.8), we find that the expression (5.9) is dominated by $q^{\varepsilon-(1-6 \Delta) / 8}$ for all $\varepsilon>0$ the implied constant depending on $\varepsilon, D_{1}, D_{2}, D_{3}$.

Remark. It is possible to avoid the use of Proposition 5.1, a rather deep result, through elementary methods (see [V2], for example) with no loss in the quality of the estimate. Here we prefer to get the desired result as quickly as possible.

We thus have (using (5.5))

$$
\begin{array}{rl}
\sum_{f}^{h} & P\left(f, \chi_{1} \chi_{2}\right) P\left(f, \chi_{3}\right) M(f) \\
& =\sum_{\mathrm{d}, \ell, n} \frac{x(\mathrm{~d} \ell) \chi_{1} * \chi_{2}(\ell n) \chi_{3}(\mathrm{~d} n)}{\mathrm{d} \ell n} V\left(\frac{\mathrm{d} n}{q^{1 / 2} D_{3}}\right) W_{\chi_{1} \chi_{2}}\left(\frac{\ell n}{q D_{1} D_{2}}\right)+\mathrm{o}(1) . \\
& =L\left(\chi_{1} \chi_{2}, 1\right) \sum_{\mathrm{d}, \ell, n} \frac{x(\mathrm{~d} \ell) \chi_{1} * \chi_{2}(\ell n) \chi_{3}(\mathrm{~d} n)}{\mathrm{d} \ell n} V\left(\frac{\mathrm{d} n}{q^{1 / 2} D_{3}}\right)+\mathrm{o}(1)
\end{array}
$$

as long as $\Delta<1 / 6$, the implied constant depending on $D_{1}, D_{2}, D_{3}, \Delta$. 
Before going to the evaluation of the more difficult term $P M^{\varepsilon}\left(\chi_{1}, \chi_{2}, \bar{\chi}_{3}\right)$, we show that we are finished calculating the third moment if any of the characters, say $\chi_{1}$, is real. By (1.1),

$$
L\left(f \cdot \chi_{1}, \frac{1}{2}\right)=\frac{1+\varepsilon_{\chi_{1}} \varepsilon_{f}}{2} L\left(f \cdot \chi_{1}, \frac{1}{2}\right)
$$

since both sides vanish if $\varepsilon_{f}=-\varepsilon_{\chi_{1}}$, so

$$
\begin{aligned}
& L\left(f \cdot \chi_{1}, \frac{1}{2}\right) L\left(f \cdot \chi_{2}, \frac{1}{2}\right) L\left(f \cdot \chi_{3}, \frac{1}{2}\right) \\
& \quad=\frac{1+\varepsilon_{\chi_{1}} \varepsilon_{f}}{2} L\left(f \cdot \chi_{1}, \frac{1}{2}\right) L\left(f \cdot \chi_{2}, \frac{1}{2}\right)\left(P\left(f, \chi_{3}\right)+\varepsilon_{\chi_{3}} \varepsilon_{f} P\left(f, \bar{\chi}_{3}\right)\right) .
\end{aligned}
$$

But, since both sides are zero unless $\varepsilon_{f}=\varepsilon_{\chi_{1}}$, we have

$$
\left(1+\varepsilon_{f} \varepsilon_{\chi_{1}}\right) \varepsilon_{\chi_{3}} \varepsilon_{f}=\left(1+\varepsilon_{f} \varepsilon_{\chi_{1}}\right) \varepsilon_{\chi_{3}} \varepsilon_{\chi_{1}}
$$

Thus

$$
\begin{aligned}
& L\left(f \cdot \chi_{1}, \frac{1}{2}\right) L\left(f \cdot \chi_{2}, \frac{1}{2}\right) L\left(f \cdot \chi_{3}, \frac{1}{2}\right) \\
& \quad=L\left(f \cdot \chi_{1}, \frac{1}{2}\right) L\left(f \cdot \chi_{2}, \frac{1}{2}\right)\left(P\left(f, \chi_{3}\right)+\varepsilon_{\chi_{1}} \varepsilon_{\chi_{3}} P\left(f, \bar{\chi}_{3}\right)\right),
\end{aligned}
$$

and both terms can be evaluated by (5.11).

\section{Evaluation of $\boldsymbol{P M}^{\varepsilon}\left(\chi_{1}, \chi_{2}, \bar{\chi}_{3}\right)$}

Note that, by appropriate labelling of characters, we may assume that $\chi_{1} \chi_{2}(-1)=1$. We define $y(n)$ by

$$
\begin{aligned}
P\left(f, \bar{\chi}_{3}\right) M(f) & =\sum_{\ell, n} \frac{\lambda_{f}(\ell n)}{(\ell n)^{1 / 2}} \sum_{(\mathrm{d}, q)=1} \frac{x(\mathrm{~d} \ell) \bar{\chi}_{3}(\mathrm{~d} n)}{\mathrm{d}} V\left(\frac{\mathrm{d} n}{q^{1 / 2} D_{3}}\right) \\
& :=\sum_{n} \frac{\lambda_{f}(n)}{n^{1 / 2}} y(n) .
\end{aligned}
$$

We have

$$
\begin{aligned}
& P M^{\varepsilon}\left(\chi_{1}, \chi_{2}, \bar{\chi}_{3}\right) \\
& \quad=-\sqrt{q} \sum_{m, n} \sum_{\frac{\chi_{1}}{} * \chi_{2}(m) y(n)}^{m^{1 / 2} n^{1 / 2}} W_{\chi_{1} \chi_{2}}\left(\frac{m}{q D_{1} D_{2}}\right) \sum_{f}^{h} \lambda_{f}(q m) \lambda_{f}(n) .
\end{aligned}
$$

We use Petersson's formula to break this into a 'diagonal' term,

$$
-\sum_{m} \frac{\chi_{1} * \chi_{2}(m) y(q m)}{m} W_{\chi_{1} \chi_{2}}\left(\frac{m}{q D_{1} D_{2}}\right)
$$

and a 'nondiagonal' term,

$$
\begin{aligned}
- & 2 \pi \sqrt{q} \sum_{c>0, c \equiv 0(q)} c^{-1} \sum_{m, n} \frac{\chi_{1} * \chi_{2}(m)}{m^{1 / 2}} W_{\chi_{1} \chi_{2}}\left(\frac{m}{q D_{1} D_{2}}\right) \frac{y(n)}{n^{1 / 2}} \times \\
& \times S(q m, n ; c) J_{1}\left(\frac{4 \pi \sqrt{q m n}}{c}\right) .
\end{aligned}
$$


By (5.4) and (5.8) the diagonal term is $\ll q^{\varepsilon}\left(1+q^{(1-6 \Delta) / 2}\right)^{-A}$ for all $\varepsilon, A>0$ and is thus negligible as long as $\Delta<1 / 6$. Thus we need only consider the non-diagonal term. Note first that the contribution from when $n$ is divisible by $q$ is $\ll q^{\varepsilon}\left(1+q^{(1-6 \Delta) / 2}\right)^{-A}$ for all $\varepsilon, A>0$, and can thus also be ignored. Thus we may assume that $(n, q)=1$. We note that $S(q m, n ; c)=0$ unless $q$ divides $c$ exactly once, in which case, writing $c=q c^{\prime}$ with $\left(c^{\prime}, q\right)=1$, we have

$S(q m, n ; c)=S\left(m, \bar{q} n ; c^{\prime}\right) S\left(0, \overline{c^{\prime}} n ; q\right)=-S\left(m, \bar{q} n ; c^{\prime}\right)$.

Thus

$$
\begin{aligned}
P M^{\varepsilon}= & \frac{2 \pi}{\sqrt{q}} \sum_{(n, q)=1} \frac{y(n)}{n^{1 / 2}} \sum_{(c, q)=1} \sum_{m} \frac{\chi_{1} * \chi_{2}(m)}{m^{1 / 2}} \frac{S(m, \bar{q} n ; c)}{c} \times \\
& \times W_{\chi_{1} \chi_{2}}\left(\frac{m}{q D_{1} D_{2}}\right) J_{1}\left(\frac{4 \pi \sqrt{m n / q}}{c}\right)+\text { Err. }
\end{aligned}
$$

By Weil's bound for Kloosterman sums and the inequality $J_{1}(x) \ll x$ we can ignore the contribution from large $c$, say $c \geqslant C$ for $C=q^{1000}$. To simplify computations, we multiply the expression by $\eta(m)$, where $\eta(x)$ is smooth, vanishing near the origin, and equal to one on $[1,+\infty)$. Note that the sum is unchanged by this step.

\subsection{APPLICATION OF POISSON'S FORMULA}

We now write $S(m, \bar{q} n ; c)$ as a sum of exponentials $e((a m+\overline{a q n}) / c)$ and apply Proposition A.2 for the function

$$
\eta(x) F(x)=\eta(x) x^{-1 / 2} W_{\chi_{1} \chi_{2}}\left(\frac{x}{q D_{1} D_{2}}\right) J_{1}\left(\frac{4 \pi \sqrt{x n / q}}{c}\right) .
$$

We can then write $P M^{\varepsilon}=P M_{\text {main }}^{\varepsilon}+P M_{\mathrm{er}}^{\varepsilon}+E r r$ with

$$
\begin{aligned}
P M_{\text {main }}^{\varepsilon}= & 2 \pi \frac{G\left(\chi_{2}\right) L\left(1, \chi_{1} \bar{\chi}_{2}\right)}{\sqrt{q}} \times \\
& \times \sum_{(n, q)=1} \frac{y(n)}{n^{1 / 2}} \sum_{\substack{c \leqslant C, D_{2} \mid c \\
(c, q)=1}} \chi_{1}\left(\frac{c}{D_{2}}\right) \frac{G\left(\chi_{2}, \bar{q} n ; c\right)}{c^{2}} \int_{0}^{\infty} \eta(x) F(x) \mathrm{d} x+ \\
& +2 \pi \frac{G\left(\chi_{1}\right) L\left(1, \bar{\chi}_{1} \chi_{2}\right)}{\sqrt{q}} \times \\
& \times \sum_{(n, q)=1} \frac{y(n)}{n^{1 / 2}} \sum_{\substack{c \leqslant C, D_{1} \mid c \\
(c, q)=1}} \chi_{2}\left(\frac{c}{D_{1}}\right) \frac{G\left(\chi_{1}, \bar{q} n ; c\right)}{c^{2}} \int_{0}^{\infty} \eta(x) F(x) \mathrm{d} x,
\end{aligned}
$$

where $G\left(\chi_{2}, \bar{q} n ; c\right)=\sum_{a(c),(a, c)=1} \chi_{2}(a) e(a \bar{q} n / c)$ and 


$$
\begin{aligned}
P M_{e r}^{\varepsilon}= & -\frac{4 \pi^{2}}{\sqrt{q}} \sum_{(n, q)=1} \frac{y(n)}{n^{1 / 2}} \sum_{\substack{c \leqslant C \\
(c, q)=1}} \sum_{m_{1}, m_{2}} \frac{R\left(m_{1}, m_{2}, \bar{q} n ; c\right)}{c c_{1} c_{2}} \times \\
& \times \int_{0}^{\infty} \eta(x) F(x) Y_{0}\left(4 \pi \sqrt{\frac{m_{1} m_{2} x}{c_{1} c_{2}}}\right) \mathrm{d} x+ \\
& +8 \pi \frac{\chi_{2}(-1)}{\sqrt{q}} \sum_{(n, q)=1} \frac{y(n)}{n^{1 / 2}} \sum_{\substack{c \leqslant C \\
(c, q)=1}} \sum_{m_{1}, m_{2}} \frac{R\left(m_{1}, m_{2},-\bar{q} n ; c\right)}{c c_{1} c_{2}} \times \\
& \times \int_{0}^{\infty} \eta(x) F(x) K_{0}\left(4 \pi \sqrt{\frac{m_{1} m_{2} x}{c_{1} c_{2}}}\right) \mathrm{d} x,
\end{aligned}
$$

where $R\left(m_{1}, m_{2}, n ; c\right)$ is the exponential sum defined in (B.1). Using Lemma B.1 we see that

$$
\begin{aligned}
P M_{e r}^{\varepsilon} \ll & q^{-1 / 2} \sum_{(n, q)=1} \frac{|y(n)|}{n^{1 / 2}} \sum_{\substack{c \leqslant C \\
(c, q)=1}} \sum_{m \geqslant 1} \tau(m) \frac{\left(\left(c_{1} c_{2} / c^{2}\right) n-q m, c\right)}{c^{2}} \times \\
& \times\left|\int_{0}^{\infty} \eta(x) F(x) Y_{0}\left(4 \pi \sqrt{\frac{m x}{c_{1} c_{2}}}\right) \mathrm{d} x\right|+ \\
& +q^{-1 / 2} \sum_{(n, q)=1} \frac{|y(n)|}{n^{1 / 2}} \sum_{\substack{c \leqslant C \\
(c, q)=1}} \sum_{m \geqslant 1} \tau(m) \frac{\left(\left(c_{1} c_{2} / c^{2}\right) n+q m, c\right)}{c^{2}} \times \\
& \times\left|\int_{0}^{\infty} \eta(x) F(x) K_{0}\left(4 \pi \sqrt{\frac{m x}{c_{1} c_{2}}}\right) \mathrm{d} x\right|,
\end{aligned}
$$

the implied constant depending only on $D_{1}, D_{2}$. We bound these terms by the methods of [KM2] 2.4.6 which gives that the inner sums over the variable $c, m$ are bounded by $\ll q^{\varepsilon-1 / 2} n^{1 / 2}$. Note in particular the singularity for

$$
n=q m\left(c_{1} c_{2} / c^{2}\right)^{-1} \geqslant q m /\left(D_{1} D_{2}\right)
$$

but from (5.8), as long as $1 / 2+3 \Delta<1$, it gives a negligable contribution. The net result is that $P M_{e r}^{\varepsilon}$ is bounded by

$$
\ll q^{\varepsilon-1} \sum_{(n, q)=1}|y(n)| \ll q^{\varepsilon-1 / 2+3 \Delta},
$$

which is admissible as long as $\Delta<1 / 6$.

\subsection{EVALUATION OF $P M_{\text {main }}^{\varepsilon}$}

Now we evaluate the two terms of $P M_{\text {main }}^{\varepsilon}$. First we remove the function $\eta(x)$ from the integral at a cost $\ll q^{-1 / 2+3 \Delta}$ (using that $J_{1}(x) \ll x$ ), and we extend the summation to $c>C$ at a negligible cost. Next we make the change of variable $y=\left(4 \pi \sqrt{n} / \sqrt{q} c D_{2}\right) x^{1 / 2}$. In the definition of $W_{\chi_{1} \chi_{2}}$ as an inverse Mellin transform 
we shift the line of integration to $\sigma=1 / 4$, switch this integral with the $x$-integral and the $c$ sum (everything is absolutely convergent) and use the identity

$$
\int_{0}^{\infty} J_{1}(y) y^{-2 s} \mathrm{~d} y=2^{-2 s} \frac{\Gamma(1-s)}{\Gamma(1+s)} .
$$

We obtain finally that the first of the two terms of $P M_{\text {main }}^{\varepsilon}$ is

$$
\begin{aligned}
& \frac{G\left(\chi_{2}\right)}{D_{2}} L\left(1, \chi_{1} \chi_{2}\right) \sum_{n} \frac{y(n)}{n} \frac{1}{2 \pi \mathrm{i}} \times \\
& \quad \times \int_{(1 / 4)} \Gamma(1-s) \Gamma(1+s) L^{(q)}\left(1+2 s, \chi_{1} \chi_{2}\right) Z(1+2 s)\left(\frac{D_{1}}{D_{2} n}\right)^{s} \frac{\mathrm{d} s}{s},
\end{aligned}
$$

where

$$
Z(s)=\sum_{(c, q)=1} \chi_{1}(c) \frac{G\left(\chi_{2}, \bar{q} n ; c D_{2}\right)}{c^{s}} .
$$

We have

$$
\begin{aligned}
G\left(\chi_{2}, \bar{q} n ; c D_{2}\right) & =\sum_{\substack{a\left(c D_{2}\right) \\
(a, c)=1}} \chi_{2}(a) e\left(\frac{a \bar{q} n}{c D_{2}}\right) \\
& =\sum_{u \mid c} \mu(u) \chi_{2}(u) \sum_{a\left(c D_{2}\right)} \chi_{2}(a) e\left(\frac{a u \bar{q} n}{c D_{2}}\right) \\
& =\sum_{\substack{u|c \\
c / u| n}} \mu(u) \chi_{2}(u) \frac{c}{u} \sum_{a\left(D_{2}\right)} \chi_{2}(a) e\left(\frac{a \bar{q} n /(c / u)}{D_{2}}\right) \\
& =G\left(\chi_{2}\right) \chi_{2}(q) \sum_{\substack{e f=n \\
e \mid c}} e \bar{\chi}_{2}(f) \mu(c / e) \chi_{2}(c / e) .
\end{aligned}
$$

We obtain that

$$
Z(s)=\sum_{\substack{e f=n \\(e, q)=1}} \chi_{1}(e) \bar{\chi}_{2}(f) e^{1-s} L^{(q)}\left(\chi_{1} \chi_{2}, s\right)^{-1} .
$$

Note that we can drop the condition $(e, q)=1$ at an admissible cost since $q \mid e$ implies that $q \mid n$, and we have already ruled out that case. Finally, up to an admissible remainder term, (6.2) equals

$$
\frac{G^{2}\left(\chi_{2}\right) \chi_{2}(q)}{D_{2}} L\left(1, \chi_{1} \bar{\chi}_{2}\right) \sum_{n} \frac{y(n)}{n} F\left(\chi_{1}, \chi_{2}, n\right),
$$

where

$$
F\left(\chi_{1}, \chi_{2}, n\right)=\frac{1}{2 \pi \mathrm{i}} \int_{(1 / 4)} \Gamma(1-s) \Gamma(1+s)\left(\frac{D_{1}}{D_{2}}\right)^{s} \sum_{e f=n} \chi_{1}(e) \bar{\chi}_{2}(f)\left(\frac{e}{f}\right)^{s} \frac{\mathrm{d} s}{s} .
$$


So, up to a remainder dominated by $\ll q^{\varepsilon-1 / 2+3 \Delta}$,

$$
\begin{aligned}
P M^{\varepsilon}=\sum_{n} \frac{y(n)}{n}\left[\frac{G^{2}\left(\chi_{2}\right) \chi_{2}(q)}{D_{2}} L\left(1, \chi_{1} \bar{\chi}_{2}\right) F\left(\chi_{1}, \chi_{2}, n\right)+\right. \\
\left.+\frac{G^{2}\left(\chi_{1}\right) \chi_{1}(q)}{D_{1}} L\left(1, \bar{\chi}_{1} \chi_{2}\right) F\left(\chi_{2}, \chi_{1}, n\right)\right] .
\end{aligned}
$$

This finishes our computation of $P M^{\S}\left(\chi_{1}, \chi_{2}, \bar{\chi}_{3}\right)$.

\subsection{END OF THE COMPUTATION}

We now combine the sums to calculate

$$
\begin{aligned}
& \sum_{f}^{h} L\left(f \cdot \chi_{1}, \frac{1}{2}\right) L\left(f \cdot \chi_{2}, \frac{1}{2}\right) \varepsilon_{f} P\left(f, \bar{\chi}_{3}\right) M(f) \\
& \quad=\sum_{f}^{h} P\left(f, \chi_{1} \chi_{2}\right) \varepsilon_{f} P\left(f, \bar{\chi}_{3}\right) M(f)+\varepsilon_{\chi_{1}} \varepsilon_{\chi_{2}} \sum_{f}^{h} P\left(f, \bar{\chi}_{1} \bar{\chi}_{2}\right) \varepsilon_{f} P\left(f, \bar{\chi}_{3}\right) M(f) .
\end{aligned}
$$

At this point, it is sufficient to compute

$$
\begin{aligned}
& {\left[\frac{G^{2}\left(\chi_{2}\right) \chi_{2}(q)}{D_{2}} L\left(1, \chi_{1} \bar{\chi}_{2}\right) F\left(\chi_{1}, \chi_{2}, n\right)+\frac{G^{2}\left(\chi_{1}\right) \chi_{1}(q)}{D_{1}} L\left(1, \bar{\chi}_{1} \chi_{2}\right) \sum_{n} F\left(\chi_{2}, \chi_{1}, n\right)\right]+} \\
& \quad+\varepsilon_{\chi_{1}} \varepsilon_{\chi_{2}}\left[\frac{G^{2}\left(\bar{\chi}_{2}\right) \bar{\chi}_{2}(q)}{D_{2}} L\left(1, \bar{\chi}_{1} \chi_{2}\right) \sum_{n} F\left(\bar{\chi}_{1}, \bar{\chi}_{2}, n\right)+\right. \\
& \left.\quad+\frac{G^{2}\left(\bar{\chi}_{1}\right) \bar{\chi}_{1}(q)}{D_{1}} L\left(1, \chi_{1} \bar{\chi}_{2}\right) \sum_{n} F\left(\bar{\chi}_{2}, \bar{\chi}_{1}, n\right)\right]
\end{aligned}
$$

By shifting the $s$ contour in (6.3) to $\sigma=-1 / 4$ we get a simple pole at $s=0$. Making the change of variable $s \rightarrow-s$, we have

$$
F\left(\chi_{1}, \chi_{2}, n\right)+F\left(\bar{\chi}_{2}, \bar{\chi}_{1}, n\right)=\chi_{1} * \bar{\chi}_{2}(n)
$$

Using the hypothesis $\chi_{1} \chi_{2}(-1)=1$ and the identity

$$
\varepsilon_{\chi_{1}} \varepsilon_{\chi_{2}}=\chi_{1} \chi_{2}(q) \frac{G\left(\chi_{1}\right)}{G\left(\bar{\chi}_{1}\right)} \frac{G\left(\chi_{2}\right)}{G\left(\bar{\chi}_{2}\right)},
$$

we find that (6.4) equals

$$
\frac{G^{2}\left(\chi_{2}\right) \chi_{2}(q)}{D_{2}} L\left(1, \chi_{1} \bar{\chi}_{2}\right) \chi_{1} * \bar{\chi}_{2}(n)+\frac{G^{2}\left(\chi_{1}\right) \chi_{1}(q)}{D_{1}} L\left(1, \bar{\chi}_{1} \chi_{2}\right) \bar{\chi}_{1} * \chi_{2}(n)
$$


Thus we have proved the following, theorem:

THEOREM 6.1. Let $\chi_{1}, \chi_{2}, \chi_{3}$ be three distinct primitive characters of conductor $D_{1}, D_{2}, D_{3}$ such that $\chi_{1} \chi_{2}(-1)=1$. Then for any $\Delta<1 / 6$,

$$
\begin{aligned}
\sum^{h} & \prod L\left(f \cdot \chi_{i}, \frac{1}{2}\right) M_{i}(f) \\
= & P M\left(\chi_{1}, \chi_{2}, \chi_{3}\right)+\varepsilon_{\chi_{1}} \varepsilon_{\chi_{2}} P M\left(\bar{\chi}_{1}, \bar{\chi}_{2}, \chi_{3}\right)+ \\
& +\varepsilon_{3} P M^{\varepsilon}\left(\chi_{1}, \chi_{2}, \bar{\chi}_{3}\right)+\varepsilon_{3} P M^{\varepsilon}\left(\chi_{2}, \chi_{1}, \bar{\chi}_{3}\right)+o(1)
\end{aligned}
$$

the implied constant depending on only on $D_{1}, D_{2}, D_{3}, \Delta$, where

$$
P M\left(\chi_{1}, \chi_{2}, \chi_{3}\right)=L\left(1, \chi_{1} \chi_{2}\right) \sum \sum_{\mathrm{d}, \ell, n} \frac{x(\mathrm{~d} \ell) \chi_{1} * \chi_{2}(\ell n) \chi_{3}(\mathrm{~d} n)}{\mathrm{d} \ell n} V\left(\frac{\mathrm{d} n}{q^{1 / 2} D_{3}}\right)
$$

and

$$
P M^{\varepsilon}\left(\chi_{1}, \chi_{2}, \bar{\chi}_{3}\right)=G^{2} \frac{\left(\chi_{2}\right) \chi_{2}(q)}{D_{2}} L\left(1, \chi_{1} \bar{\chi}_{2}\right) \sum \sum_{d, \ell, n} \frac{x(d \ell) * \bar{\chi}_{2}(\ell n) \bar{\chi}_{3}(d n)}{d \ell n} V\left(\frac{d n}{q^{1 / 2} D_{3}}\right)
$$

\section{The Fourth Moment}

In this section and the following, $\chi$ is a complex primitive character of conductor $D$, such that $\chi^{2}$ is also primitive of conductor $D>1$. We wish to calculate asymptotics for the fourth moment

$$
\sum^{h}\left|L\left(f \cdot \chi, \frac{1}{2}\right)\right|^{4}|M(f)|^{4} .
$$

We start by calculating a precise asymptotic expansion for the twisted fourth moment

$$
M_{4}(\ell):=\sum_{f \in S_{2}^{*}(q)}^{h}|L(f \cdot \chi, 1 / 2)|^{4} \lambda_{f}(\ell)
$$

We can assume in what follows that $\ell$ is coprime with $q D$.

PROPOSITION 7.1. Let $\chi$ be a nontrivial complex character of conductor $D$ squarefree, we assume also that $\chi^{2}$ is also primitive. For any prime $q$, and any $\ell<q$ coprime with $q D$, an any $\varepsilon>0$ we have

$$
M_{4}(\ell)=M^{D}(\ell)+M^{O D}(\ell)+M^{O O D}(\ell)+O_{\varepsilon, D}\left(q^{\varepsilon}\left(\ell^{3 / 4} q^{-1 / 12}+\ell^{17 / 8} q^{-1 / 4}+\ell q^{-1 / 6}\right)\right)
$$

where $M^{D}(\ell)+M^{O D}(\ell)$ is defined in (7.9) and $M^{O O D}(\ell)$ is defined in (8.14).

This proposition is a variant of Theorem 1.2 of [KMV2] and is proved in essentially the same way. Since the analysis of the error terms is exactly the same as in [DFI2, KMV2] (because $D$ is fixed) we will almost entirely skip it to concentrate on the evaluation of the main terms. In particular when we refer to an expression 
as being an 'admissible error term' we mean that the contribution of this expression to the whole $M_{4}(\ell)$ can be bounded by $O_{\varepsilon, D}\left(q^{\varepsilon}\left(\ell^{3 / 4} q^{-1 / 12}+\ell^{17 / 8} q^{-1 / 4}+\ell q^{-1 / 6}\right)\right)$.

To simplify notation we define the twisted divisor function

$$
\tau_{\chi}(n):=\chi * \bar{\chi}(n)=\sum_{a b=n} \chi(a) \bar{\chi}(b)
$$

it satisfies

$$
\tau_{\chi}(m) \tau_{\chi}(n)=\sum_{\mathrm{d} \mid(m, n)} \varepsilon_{D}(d) \tau_{\chi}\left(\frac{m n}{\mathrm{~d}^{2}}\right), \tau_{\chi}(m n)=\sum_{\mathrm{d} \mid(m, n)} \varepsilon_{D}(\mathrm{~d}) \mu(\mathrm{d}) \tau_{\chi}\left(\frac{m}{\mathrm{~d}}\right) \tau_{\chi}\left(\frac{n}{\mathrm{~d}}\right)
$$

Let $G(s)$ be a real even polynomial such that $G(0)=1$ and $G$ vanishes at order at least 2 at $s=1,2,3$ (this simplifies some technical aspects of the computation). We have

$$
|L(f \otimes \chi, 1 / 2)|^{2}=2 \sum_{n} \frac{\tau_{\chi}(n)}{\sqrt{n}} \lambda_{f}(n) W\left(\frac{n}{q D^{2}}\right)
$$

with

$$
W(y):=\frac{1}{2 \pi \mathrm{i}} \int_{(3)} G(s) \Gamma^{2}(1+s) \zeta^{(q D)}(1+2 s)\left(4 \pi^{2} y\right)^{-s} \frac{\mathrm{d} s}{s} .
$$

We have by (2.1)

$$
\begin{aligned}
M_{4}(\ell)= & \sum_{d e=\ell} d^{-1 / 2} \sum_{a b=d} \frac{\mu(a)}{a^{1 / 2}} \tau_{\chi}(b) \sum_{m, n} \frac{\tau_{\chi}(m) \tau_{\chi}(n)}{(m n)^{1 / 2}} W\left(\frac{m}{q D^{2}}\right) \times \\
& \times W\left(\frac{a d n}{q D^{2}}\right) \sum_{f}^{h} \lambda_{f}(m) \lambda_{f}(a e n) .
\end{aligned}
$$

Using (2.3), we split this into

$$
M_{4}(\ell)=M^{D}(\ell)+M^{N D}(\ell)
$$

with

$$
\begin{aligned}
M_{4}^{D}(\ell)= & \sum_{d e=\ell} \frac{1}{d^{1 / 2}} \sum_{a b=d} \frac{\mu(a)}{a^{1 / 2}} \tau_{\chi}(b) \sum_{n} \frac{\tau_{\chi}(a e n) \tau_{\chi}(n)}{(a e)^{1 / 2} n} W\left(\frac{a e n}{q D^{2}}\right) W\left(\frac{a d n}{q D^{2}}\right), \\
M_{4}^{N D}(\ell)= & -2 \pi \sum_{d e=\ell} \frac{1}{d^{1 / 2}} \sum_{a b=d} \frac{\mu(a)}{\sqrt{a}} \tau_{\chi}(b) \sum_{c \equiv 0(q)} \frac{1}{c} \sum_{m, n} \frac{\tau_{\chi}(m) \tau_{\chi}(n)}{\sqrt{m n}} W\left(\frac{m}{q D^{2}}\right) \times \\
& \times W\left(\frac{a d n}{q D^{2}}\right) S(m, a e n ; c) J_{k-1}\left(\frac{4 \pi \sqrt{a e m n}}{c}\right) .
\end{aligned}
$$

We have by (7.2)

$$
M^{D}(\ell)=\frac{1}{\ell^{1 / 2}} \sum_{d e=\ell} \sum_{n} \frac{\tau_{\chi}(e n) \tau_{\chi}(d n)}{n} W\left(\frac{e n}{q D^{2}}\right) W\left(\frac{d n}{q D^{2}}\right) .
$$


Before evaluating $M^{N D}(\ell)$, we make a smooth dyadic partition of unity on the variables $x$ and $n$ by introducing (as with the third moment) $\eta(x)$, which vanishes near the origin and is one on $[1,+\infty)$. We decompose $\eta$ as $\eta(x)=\sum_{M \geqslant 1} \eta_{M}(x)$, a sum of smooth functions $\eta_{M}$ which are compactly supported on $[M / 2,2 M]$ with derivatives satisfying $x^{j} \eta^{(j)}(x) \ll_{j} 1$ for any $j$. We set

$$
D(x, n):=\frac{1}{\sqrt{x n}} W\left(\frac{x}{q D^{2}}\right) W\left(\frac{a d n}{q D^{2}}\right)
$$

and we use the generic notation $F_{M, N}(x, n)$ or $F(x, n)$ to designate a function of the form

$$
F_{M, N}(x, n):=\eta_{M}(x) \eta_{N}(n) D(x, n) .
$$

We thus have

$$
\begin{aligned}
M_{4}^{N D}(\ell)= & -2 \pi \sum_{d e=\ell} \frac{1}{d^{1 / 2}} \sum_{a b=d} \frac{\mu(a)}{\sqrt{a}} \tau_{\chi}(b) \times \\
& \times \sum_{M, N} \sum_{c \equiv 0(q)} \frac{1}{c} \sum_{m, n} \tau_{\chi}(m) \tau_{\chi}(n) F_{M, N}(m, n) S(m, \text { aen } ; c) J_{1}\left(\frac{4 \pi \sqrt{a e m n}}{c}\right) .
\end{aligned}
$$

We note that we can remove the contributions from $M+N \geqslant q^{1+\varepsilon}$ and then from $c \geqslant C$ (where $C=\min \left(q^{7 / 6}, q^{2 / 3} M^{1 / 2}\right)$ ) at an admissible cost, using (5.4) and Proposition 5.1. We apply Proposition A.2 and get

$$
\begin{aligned}
& c \sum_{m} \tau_{\chi}(m) F(m, n) S(m, \text { aen } ; c) \\
& =\delta_{D \mid c} 2 \Re e\left(\chi\left(\frac{c}{D}\right) G(\chi, \text { aen } ; c) G(\bar{\chi}) L\left(1, \chi^{2}\right)\right) \int_{0}^{\infty} F(x, n) \mathrm{d} x+ \\
& \quad+\sum_{ \pm} \chi(\mp 1) \sum_{m \geqslant 1} T(m, \mp a e n ; c) \hat{F}^{ \pm}(m, n)
\end{aligned}
$$

where

$$
\begin{aligned}
& \hat{F}^{ \pm}(y, n):=-2 \pi \int_{0}^{\infty} F(x, n) J_{1}\left(\frac{4 \pi \sqrt{x a e n}}{c}\right) K^{ \pm}\left(4 \pi \frac{\sqrt{x y}}{[c, D]}\right) \mathrm{d} x, \\
& K^{-}(x):=-2 \pi Y_{0}(x), \quad K^{+}(x):=4 K_{0}(x), \\
& T(m, n ; c):=\sum_{m_{1} m_{2}=m} R\left(m_{1}, m_{2}, n ; c\right)
\end{aligned}
$$

and

$$
R\left(m_{1}, m_{2}, n ; c\right)=\frac{c}{[c, D]^{2}} \sum_{\substack{x(c) \\(x, c)=1}} e\left(\frac{n \bar{x}}{c}\right) \sum_{r_{1}, r_{2}([c, D])} \chi\left(r_{1}\right) \bar{\chi}\left(r_{2}\right) e\left(\frac{m_{1} r_{1}+m_{2} r_{2}}{[c, D]}+\frac{r_{1} r_{2} x}{c}\right)
$$


is the sum studied in Section B.1 of the Appendix. The contribution of the first term can be shown negligible in exactly the same way as in [DFI2] p. 229 or [KMV2] 3.3, the only adjustment being powers of $D$ to consider, so we will not cover it in detail here. This leaves the sum over $m$, which we break into two cases.

\subsection{EVALUATION OF THE OFF-DIAGONAL TERM}

We first consider the special case $m=(D /(c, D))^{2}$ aen (the 'off-diagonal' term), specifically its contribution to the ' + ' part of the sum in (7.6). For notational convenience, we let $D_{3}=D /(c, D)$, so that

$$
\begin{aligned}
M_{4}^{O D}(\ell)= & \sum_{d e=\ell} \frac{1}{d^{1 / 2}} \sum_{a b=d} \frac{\mu(a)}{\sqrt{a}} \tau_{\chi}(b) \times \\
& \times \sum_{\substack { M, N \\
\begin{subarray}{c}{c=0(q) \\
c \leqslant c{ M , N \\
\begin{subarray} { c } { c = 0 ( q ) \\
c \leqslant c } }\end{subarray}} \frac{1}{c} \sum_{n} \tau_{\chi}(n) T\left(a e n D_{3}^{2}, \text { aen } ; c\right) \hat{F}_{M, N}^{-}\left(D_{3}^{2} \text { aen }, n\right) .
\end{aligned}
$$

From (B.4), we see that $T\left(a e n D_{3}^{2}, a e n ; c\right)=0$ unless $D_{3}=1$, so $D \mid c$. Combining (B.4) with (B.3), we find that

$$
T(\text { aen }, \text { aen } ; c)=\tau_{\chi}(\text { aen }) \phi\left(\frac{c}{D_{1}}\right) \mu\left(D_{1}\right)
$$

where $D_{1}$ is the largest divisor of $(D, c)$ such that $\left(D_{1}, c / D_{1}\right)=1$. As in [KMV2] 3.5, we can drop the constrains $c \leqslant C$ and $M+N \leqslant q^{1+\varepsilon}$ at an admissible cost. At this point (7.7) is almost exactly identical to the off-diagonal terms studied in [KMV2], Section 3.6, the only difference being the replacement of $\tau$ by $\tau_{\chi}$ and $\phi(c)$ by $\phi\left(c / D_{1}\right) \mu\left(D_{1}\right)$. Thus we may follow the steps of that paper precisely, using the calculation

$$
\sum_{q D \mid c} \frac{\phi\left(\frac{c}{D_{1}}\right) \mu\left(D_{1}\right)}{c^{1+2 s}}=\frac{\phi(q)}{q^{1+2 s}} D^{1-2 s} \frac{\zeta(2 s)}{\zeta^{(q D)}(1+2 s) \zeta^{(D)}(1-2 s)},
$$

and the functional equation

$$
\zeta(2 s) \Gamma(s)=\pi^{2 s-1 / 2} \zeta(1-2 s) \Gamma(1 / 2-s) .
$$

to get an off-diagonal term of

$$
\begin{aligned}
& \sum_{d e=\ell} \frac{1}{\ell^{1 / 2}} \sum_{a b=d} \frac{\mu(a)}{a} \tau_{\chi}(b) \sum_{n} \frac{\tau_{\chi}(a e n)}{n} W\left(\frac{a d n}{q D^{2}}\right) \times \\
& \quad \times \frac{1}{2 \pi \mathrm{i}} \int_{(3)} G(s) \Gamma^{2}(1-s) \frac{\varphi(q)}{q} \zeta^{(D)}(1-2 s)\left(\frac{4 \pi^{2} a e n}{q D^{2}}\right)^{s} \frac{\mathrm{d} s}{s} .
\end{aligned}
$$


We make the change of variable $s^{\prime}=-s$, then shift the resulting contour to the right up to Res $=3$, passing a pole at $s^{\prime}=0$. After rearranging the $a, b, e$ sums and combining with the the diagonal term (7.3), we obtain that

$$
\begin{aligned}
M^{D}(\ell)+M^{O D}(\ell)= & \frac{\varphi(q)}{q} \frac{4}{\ell^{1 / 2}} \sum_{d e=\ell} \sum_{n} \frac{\tau_{\chi}(d n) \tau_{\chi}(e n)}{n} W\left(\frac{d n}{q D^{2}}\right) \operatorname{Res}_{s=0} \times \\
& \times \frac{G(s) \Gamma^{2}(1+s)\left(q D^{2}\right)^{s}}{s\left(4 \pi^{2} e n\right)^{s}} \zeta^{(D)}(1+2 s)+ \\
& +\frac{4}{\ell^{1 / 2}} \sum_{d e=\ell} \sum_{n} \frac{\tau_{\chi}(d n) \tau_{\chi}(e n)}{n} W\left(\frac{d n}{q D^{2}}\right) W^{*}\left(\frac{e n}{q D^{2}}\right)
\end{aligned}
$$

up to and admissible error term with

$$
W^{*}(y)=\frac{1}{2 \pi \mathrm{i}} \int_{(3)} G(s) \Gamma^{2}(1+s)\left(\zeta^{(q D)}(1+2 s)-\frac{\varphi(q)}{q} \zeta^{(D)}(1+2 s)\right)\left(4 \pi^{2} y\right)^{-s} \frac{\mathrm{d} s}{s} .
$$

Shifting the contour to Res $=\varepsilon$ gives $W^{*}(y) \ll_{\varepsilon}(y q)^{\varepsilon} q^{-1}$, so this second sum is negligible.

\section{The Off-Off Diagonal Term}

The 'off-off diagonal' term, which we denote $M^{O O D}(\ell)$, is the contribution from $M^{N D}(\ell)$ when $m \neq D_{3}^{2}$ aen. As in [KMV2], its evaluation is by far the most difficult and technical. We start with a notational adjustment: to any $c>0$ we associate the decomposition $D=D_{1} D_{2}$ and the divisor $D_{3} \mid D_{2}$ uniquely defined by the properties

$$
c=c^{\prime} D_{1}, \quad\left(c^{\prime}, D_{1} D_{3}\right)=1, \quad\left(D_{2} / D_{3}\right)^{2} \mid c^{\prime} .
$$

Note that this is slightly different than the decomposition in Appendix B, we have adjusted it because here the only important distinction is between primes dividing $c$ once and dividing it any other number of times. There is no change in the meaning of $D_{3}$, but what we now call $D_{2}$ is called $D_{2} D_{3}$ in the appendix.

We first write $M^{O O D}(\ell)$ as a sum over $M$ and $N$ of terms of the form

$$
\begin{aligned}
M_{M, N}^{O O D}(\ell)=-2 \pi & \sum_{d e=\ell} \frac{1}{d^{1 / 2}} \sum_{a b=d} \frac{\mu(a)}{\sqrt{a}} \tau_{\chi}(b) \times \\
& \times \sum_{c \equiv 0(q)} \frac{1}{c^{2}} \sum_{ \pm} \chi(\mp) \sum_{h \neq 0} \sum_{m \pm D_{3}^{2} a e n=h} T(m, \mp a e n ; c) F_{M, N}^{ \pm}(m, n) .
\end{aligned}
$$

Using Equation (B.4), we see that the off-off-diagonal term is provided by the sum over $a, c, e, M$, and $N$ of

$$
\begin{aligned}
\chi_{D_{3}}(-1) \sum_{v \in\{ \pm 1\}} \chi(-v) \sum_{h \neq 0} r\left(h ; c^{\prime}\right) \times \\
\quad \times\left[\sum_{m_{1} m_{2}+v D_{3}^{2} \text { aen }=h} \bar{\chi}_{D_{2}}\left(m_{1}\right) \chi_{D_{2}}\left(m_{2}\right) \tau_{\chi}(n) R\left(m_{1}, m_{2},- \text { vaen } ; D_{1}\right) \hat{F}_{M, N}^{ \pm}\left(m_{1} m_{2}, n\right)\right]
\end{aligned}
$$


with

$$
R\left(m_{1}, m_{2}, n ; D_{1}\right)=\frac{1}{D_{1}} \sum_{x\left(D_{1}\right)}^{*} \sum_{r_{1}, r_{2}\left(D_{1}\right)} \chi_{D_{1}}\left(r_{1}\right) \bar{\chi}_{D_{1}}\left(r_{2}\right) e\left(\frac{m_{1} r_{1}+m_{2} r_{2}+D_{3}\left(r_{1} r_{2} x+n \bar{x}\right)}{D_{1}}\right)
$$

Since $q \mid c^{\prime}$, we can decompose $r\left(h ; c^{\prime}\right)$ as

$$
r\left(h ; c^{\prime}\right)=\sum_{\substack{g c^{\prime \prime}=c^{\prime},(q, g)=1 \\ c^{\prime \prime} \mid h}} \mu(g) c^{\prime \prime}+\sum_{\substack{g c^{\prime \prime}=c^{\prime}, q\left|g \\ c^{\prime \prime}\right| h}} \mu(g) c^{\prime \prime}:=r^{m}\left(h ; c^{\prime}\right)+r^{e}\left(h, c^{\prime}\right) .
$$

One can show, exactly as in [KMV2] 4.4, that the $r^{e}(h ; c)$ portion contributes an admissible error term so in the expression (8.1) we may replace $r\left(h, c^{\prime}\right)$ by $r^{m}\left(h ; c^{\prime}\right)$. We compute now the sum over the $m_{1}, m_{2}, n$ variables. The treatment of remainder terms and the choice of parameters in what is to come is the same as in [DFI2] and we refer to that paper (and also to [DFI1]). We apply the $\delta$-symbol method [DFI1] to detect the condition $m_{1} m_{2} \pm D_{3}^{2} a e n=h$, giving

$$
\begin{aligned}
\sum_{m_{1} m_{2}+v D_{3}^{2} a e n=h} \cdots= & \sum_{1 \leqslant r<2 R} \frac{1}{D_{1}} \sum_{d(r)}^{*} e\left(\frac{-d h}{r}\right) \sum_{r_{1}, r_{2}, x\left(D_{1}\right)}^{*} \chi_{D_{1}}\left(r_{1}\right) \bar{\chi}_{D_{1}}\left(r_{2}\right) e\left(\frac{r_{1} r_{2} x}{D_{1}}\right) \times \\
& \times \sum_{m_{1}, m_{2}} \bar{\chi}_{D_{2}}\left(m_{1}\right) \chi_{D_{2}}\left(m_{2}\right) e\left(\frac{m_{1} r_{1}+m_{2} r_{2}}{D_{1}}+\frac{d m_{1} m_{2}}{r}\right) \times \\
& \times \sum_{n} \tau_{\chi}(n) e\left(v D_{3}^{2} a e\left(\frac{d}{r}-\frac{\bar{x}}{D_{1}}\right) n\right) E^{v}\left(m_{1} m_{2}, n\right)
\end{aligned}
$$

where $R$ is chosen depending on $M, N$ and

$$
E^{v}(x, y)=\hat{F}^{v}(x, y) \Delta_{r}\left(x+v D_{3}^{2} a e y-h\right) .
$$

Now we perform Poisson summation using Proposition A.1 of Appendix A: we obtain

$$
\sum_{m_{1}, m_{2}, n} \sum_{\cdots} \cdots \operatorname{Res}_{s=t=0} \frac{\widehat{E}^{v}(1+s, 1+t)}{[r, D]^{2(s+t)}}\left(\frac{I_{0}}{s^{2}}+\frac{1}{s}\left(I^{-}+I^{+}\right)\right)\left(\frac{I I_{0}}{t^{2}}+\frac{\chi(v)}{t}\left(I I^{-}+I I^{+}\right)\right)+\mathrm{Err},
$$

where

$$
\begin{aligned}
& \widehat{E}^{v}(1+s, 1+t)=\iint_{0}^{+\infty} E^{v}(x, y) x^{s} y^{t} \mathrm{~d} x \mathrm{~d} y \\
& I_{0}=I_{0}\left(d, r_{1}, r_{2}\right)=\frac{1}{[r, D]^{2}} \sum_{s_{1}, s_{2}([r, D])} \bar{\chi}_{D_{2}}\left(s_{1}\right) \chi_{D_{2}}\left(s_{2}\right) e\left(\frac{r_{1} s_{1}+r_{2} s_{2}}{D_{1}}+\frac{d s_{1} s_{2}}{r}\right)
\end{aligned}
$$




$$
\begin{aligned}
& I I_{0}=I I_{0}(d, x)=\frac{1}{[r, D]^{2}} \sum_{s_{1}, s_{2}([r, D])} \bar{\chi}\left(s_{1}\right) \chi\left(s_{2}\right) e\left(D_{3}^{2} a e\left(\frac{d}{r}-\frac{\bar{x}}{D_{1}}\right) s_{1} s_{2}\right), \\
& I^{-}=I^{-}\left(d, r_{1}, r_{2}\right)=\frac{1}{[r, D]^{2}} \sum_{s_{1}, s_{2}([r, D])} \bar{\chi}_{D_{2}}\left(s_{1}\right) \chi_{D_{2}}\left(s_{2}\right) e\left(\frac{r_{1} s_{1}+r_{2} s_{2}}{D_{1}}+\frac{d s_{1} s_{2}}{r}\right)\left(-\frac{\Gamma^{\prime}}{\Gamma}\left(\frac{s_{1}}{[r, D]}\right)\right), \\
& I I^{-}=I I^{-}(d, x)=\frac{1}{[r, D]^{2}} \sum_{s_{1}, s_{2}([r, D])} \bar{\chi}\left(s_{1}\right) \chi\left(s_{2}\right) e\left(D_{3}^{2} a e\left(\frac{d}{r}-\frac{\bar{x}}{D_{1}}\right) s_{1} s_{2}\right)\left(-\frac{\Gamma^{\prime}}{\Gamma}\left(\frac{s_{1}}{[r, D]}\right)\right)
\end{aligned}
$$

and $I^{+}, I I^{+}$are defined similarly with $-\Gamma^{\prime} / \Gamma\left(s_{2} /[r, D]\right)^{\star}$. The portion called ' $E r r$ ' is the sum of 8 more terms (see (A.2)) involving sums of the (discrete) Fourier transforms of the functions

$$
\begin{aligned}
& \left(s_{1}, s_{2}\right) \rightarrow \bar{\chi}_{D_{2}}\left(s_{1}\right) \chi_{D_{2}}\left(s_{2}\right) e\left(\frac{s_{1} r_{1}+s_{2} r_{2}}{D_{1}}+\frac{d s_{1} s_{2}}{r}\right), \\
& \left(s_{1}, s_{2}\right) \rightarrow \bar{\chi}\left(s_{1}\right) \chi\left(s_{2}\right) e\left(v D_{3}^{2} a e\left(\frac{d}{r}-\frac{\bar{x}}{D_{1}}\right) s_{1} s_{2}\right),
\end{aligned}
$$

weighted by certain Bessel transforms of $E^{v}$. Summing back over the variables $d, r_{1}, r_{2}, x$ one obtains sums of Kloosterman sums to which one applies Weil's bound. The details work precisely as in [DFI2], so we skip the rather tedious calculations involved. This shows, as in [DFI2] p. 229-231, that Err contributes an admissible remainder term to the fourth moment, so that the main term comes from the contribution of the pole at $s=t=1$ of the Hurwitz zeta functions.

\subsection{COMPUTATION OF $I_{0}\left(d, r_{1}, r_{2}\right)$}

Since $\chi^{2}$ is primitive, either $\chi_{D_{2}}^{2}$ is nontrivial or $D_{2}=1$. Summing over $s_{2}$ we see that $I_{0}\left(d, r_{1}, r_{2}\right)$ is zero unless $D_{2}=1$ and $D \mid r$. In this case the sum becomes

$$
I_{0}\left(d, r_{1}, r_{2}\right)=\frac{1}{r^{2}} \sum_{s_{1}, s_{2}(r)} e\left(\frac{r_{1} s_{1}+r_{2} s_{2}}{D}+\frac{d s_{1} s_{2}}{r}\right)=\frac{1}{r} e\left(-\frac{\bar{d} r^{\prime} r_{1} r_{2}}{D}\right)
$$

with $r=r^{\prime} D$.

\subsection{COMPUTATION OF $I I_{0}(d, x)$}

Summing over $s_{2}$, then over $s_{1}$, we find that $I I_{0}$ is zero unless $D_{1} \| r$ and $\chi_{D_{1}}^{2}$ is trivial. The same holds for $D_{2}$ and $\chi_{D_{2}}^{2}$, so, since $\chi_{D}$ is assumed non-trivial, we have

$$
I I_{0}(d, x)=0 .
$$

\footnotetext{
${ }^{\star}$ Note that in order to factor out the term $\chi(v)$ we have (implicitly) made the changes of variables $s_{2}^{\prime}=v s_{2}$ for $I I^{-}$and $s_{1}^{\prime}=v s_{1}$ for $I I^{+}$, so that these expressions are now independent of $v$.
} 


\subsection{COMPUTATION OF $I^{-}\left(d, r_{1}, r_{2}\right)$}

Once again, the sum is zero unless $D \mid r$ and $(D, r / D)=1$. Let $r=D r^{\prime}$. Decomposing $s_{2}$ as $s_{2}=s_{2}^{\prime}+D t$, with $0 \leqslant t<r^{\prime}$, we get $s_{1} \equiv 0\left(r^{\prime}\right)$ and

$$
I^{-}\left(d, r_{1}, r_{2}\right)=\frac{\bar{\chi}_{D_{2}}\left(r^{\prime}\right)}{r^{\prime} D^{2}} \sum_{s_{1}, s_{2}(D)} \bar{\chi}_{D_{2}}\left(s_{1}\right) \chi_{D_{2}}\left(s_{2}\right) e\left(\frac{D_{2} r^{\prime} r_{1} s_{1}+D_{2} r_{2} s_{2}+d s_{1} s_{2}}{D}\right)\left(-\frac{\Gamma^{\prime}}{\Gamma}\left(\frac{s_{1}}{D}\right)\right) .
$$

The $s_{2}$ sum is now a Gauss sum equalling

$$
D_{1} \bar{\chi}_{D_{2}}\left(\bar{D}_{1} d s_{1}\right) G\left(\chi_{D_{2}}\right) \delta_{s_{1}=-\bar{d} D_{2} r_{2}\left(D_{1}\right)},
$$

so

$$
I^{-}\left(d, r_{1}, r_{2}\right)=\frac{\bar{\chi}_{D_{2}}\left(\bar{D}_{1} d r^{\prime}\right)}{r^{\prime} D_{2} D} G\left(\chi_{D_{2}}\right) \sum_{\substack{s_{1}(D) \\ s_{1} \equiv-\bar{d} D_{2} r_{2}\left(D_{1}\right)}} \bar{\chi}_{D_{2}}^{2} e\left(\frac{r^{\prime} r_{1} s_{1}}{D_{1}}\right)\left(s_{1}\right)\left(-\frac{\Gamma^{\prime}}{\Gamma}\left(\frac{s_{1}}{D}\right)\right) .
$$

Since $-\bar{d} D_{2} r_{2}$ is coprime with $D_{1}$, the exponential term is constant, and we can detect the congruence through multiplicative characters $\psi$ of modulus $D_{1}$, namely

$$
\sum_{\substack{s_{1}(D) \\ s_{1} \equiv-\bar{d} D_{2} r_{2}\left(D_{1}\right)}}=\frac{1}{\varphi\left(D_{1}\right)} \sum_{\psi\left(D_{1}\right)} \bar{\psi}\left(-\bar{d} D_{2} r_{2}\right) \sum_{1 \leqslant s_{1} \leqslant D} \psi\left(s_{1}\right) \bar{\chi}_{D_{2}}^{2}\left(s_{1}\right)\left(-\frac{\Gamma^{\prime}}{\Gamma}\left(\frac{s_{1}}{D}\right)\right) .
$$

Using (A.9) we obtain

$$
\begin{aligned}
I^{-}\left(d, r_{1}, r_{2}\right)= & \frac{\bar{\chi}_{D_{2}}\left(\bar{D}_{1} d r^{\prime}\right)}{r^{\prime} D_{2}} e\left(-\frac{r_{1} r_{2} r^{\prime} D_{2} \bar{d}}{D_{1}}\right) \frac{G\left(\chi_{D_{2}}\right)}{\varphi\left(D_{1}\right)} \times \\
& \times \sum_{\psi\left(D_{1}\right)} \bar{\psi}\left(-\bar{d} D_{2} r_{2}\right) \operatorname{Res}_{s=0} D^{s} L\left(\psi \bar{\chi}_{D_{2}}^{2}, 1+s\right) .
\end{aligned}
$$

The same holds for $I^{+}\left(d, r_{1}, r_{2}\right)$ except that $\chi$ and $\bar{\chi}$ exchange places.

\subsection{COMPUTATION OF $I I^{-}(d, x)$}

Summing over $s_{2}$ shows that the sum is zero unless $D_{2} \| r$, and more generally we see that the sum is zero unless every prime factor of $(r, D)$ divides $r$ exactly once. Thus we may factor $r$ into $r=r^{\prime}(r, D)$ with $\left(r^{\prime}, D\right)=1$. Summing over $s_{2}=s_{2}^{\prime}+D t$ with $0 \leqslant t<r^{\prime}$ gives zero unless $s_{1} \equiv 0$ modulo $r^{\prime} /\left(a e, r^{\prime}\right)$, so

$$
\begin{aligned}
I I^{-}(d, x)= & \frac{\bar{\chi}\left(r^{\prime} /\left(a e, r^{\prime}\right)\right)}{r^{\prime} D^{2}} \sum_{\substack{s_{1}\left(D\left(a e, r^{\prime}\right)\right) \\
s_{2}(D)}} \bar{\chi}\left(s_{1}\right) \chi\left(s_{2}\right) e\left(D_{3}^{2} \frac{a e}{\left(a e, r^{\prime}\right)}\left(\frac{d}{(D, r)}-\frac{r^{\prime} \bar{x}}{D_{1}}\right) s_{1} s_{2}\right) \times \\
& \times\left(-\frac{\Gamma^{\prime}}{\Gamma}\left(\frac{s_{1}}{D\left(a e, r^{\prime}\right)}\right)\right)
\end{aligned}
$$


The $s_{2}$ sum now equals

$$
G\left(\chi, D_{3}^{2} \frac{a e}{\left(a e, r^{\prime}\right)}\left(d \frac{D}{(D, r)}-D_{2} r^{\prime} \bar{x}\right) s_{1} ; D\right)=\bar{\chi}\left(D_{3}^{2} \frac{a e}{\left(a e, r^{\prime}\right)}\left(d \frac{D}{(D, r)}-D_{2} r^{\prime} \bar{x}\right) s_{1}\right) G(\chi)
$$

note that this is zero unless $D_{3}=1$, so $D \mid c$. Using (A.9),

$$
I I^{+}(d, x)=\left(a e, r^{\prime}\right) \frac{\chi\left(\frac{a e r^{\prime}}{\left(a e, r^{\prime}\right)^{2}}\left(d \frac{D}{(D, r)}-D_{2} r^{\prime} \bar{x}\right)\right)}{r^{\prime} D} G(\bar{\chi}) L\left(\chi^{2}, 1\right) .
$$

Again, the same applies for $I I^{-}(d, x)$ except that $\chi$ and $\bar{\chi}$ exchange places.

\subsection{COMPUTATION OF THE CROSS-TERMS}

Next we need to compute

$$
\begin{aligned}
A(h, c ; r):= & \frac{1}{D_{1}} \sum_{d(r)}^{*} e\left(\frac{-d h}{r}\right) \times \\
& \times \sum_{r_{1}, r_{2}, x\left(D_{1}\right)}^{*} \chi_{D_{1}}\left(r_{1}\right) \bar{\chi}_{D_{1}}\left(r_{2}\right) e\left(\frac{r_{1} r_{2} x}{D_{1}}\right) I_{0}\left(d, r_{1}, r_{2}\right) \sum_{ \pm} I I^{ \pm}(d, x) \\
B(h, c ; r):= & 1 / D_{1} \sum_{d(r)}^{*} e\left(\frac{-d h}{r}\right) \times \\
& \times \sum_{r_{1}, r_{2}, x\left(D_{1}\right)}^{*} \chi_{D_{1}}\left(r_{1}\right) \bar{\chi}_{D_{1}}\left(r_{2}\right) e\left(\frac{r_{1} r_{2} x}{D_{1}}\right) \sum_{ \pm} I^{ \pm}\left(d, r_{1}, r_{2}\right) \sum_{ \pm} I^{ \pm}(d, x) .
\end{aligned}
$$

By Section 8.4, these terms are zero unless $D_{3}=1$, and that $c$ only affects $A(h, c ; r)$ and $B(h, c ; r)$ through the decomposition of $D$ into $D_{1}$ and $D_{2}$.

\subsubsection{Computation of $A(h, c ; r)$}

As seen in Section 8.1, $A(h, c ; r)$ is zero unless $D_{1}=D$ and $D \mid r$, so the sum over $r_{1}$, $r_{2}$ is

$$
\sum_{r_{1}, r_{2}(D)} \chi\left(r_{1}\right) \bar{\chi}\left(r_{2}\right) e\left(\frac{r_{1} r_{2}\left(x-\bar{d} r^{\prime}\right)}{D}\right)=0
$$

since $\chi^{2}$ is nontrivial. So $A(h, c ; r)=0$.

\subsubsection{Computation of $B(h, c ; r)$}

By Section 8.3, $B(h, c ; r)$ is zero unless $r=D r^{\prime}$ with $\left(r^{\prime}, D\right)=1$. For notational purposes, we let $r(1):=r_{1}, r(-1):=r_{2}$, so that for $\varepsilon, \varepsilon^{\prime} \in\{-1,1\}$, 


$$
\begin{aligned}
I^{\varepsilon}\left(d, r_{1}, r_{2}\right)= & \frac{\chi_{D_{2}}^{\varepsilon}\left(\bar{D}_{1} r^{\prime} d\right)}{r^{\prime} D_{2}} G\left(\bar{\chi}_{D_{2}}^{\varepsilon}\right) e\left(-\frac{r_{1} r_{2} r^{\prime} D_{2} \bar{d}}{D_{1}}\right) \frac{1}{\varphi\left(D_{1}\right)} \times \\
& \times \sum_{\psi\left(D_{1}\right)} \bar{\psi}\left(-D_{2} \bar{d} r(\varepsilon)\right) \operatorname{Res}_{s=0} D^{s} L\left(\psi \chi_{D_{2}}^{2 \varepsilon} 1+s\right), \\
I I^{\varepsilon^{\prime}}(d, x)= & \left(a e, r^{\prime}\right) \frac{\chi^{\varepsilon^{\prime}}\left(\frac{a e r^{\prime}}{\left(a e, r^{\prime}\right)^{2}}\left(d-D_{2} r^{\prime} \bar{x}\right)\right)}{r} G\left(\bar{\chi}^{\varepsilon^{\prime}}\right) L\left(\chi^{2 \varepsilon^{\prime}}, 1\right) .
\end{aligned}
$$

Thus we can define

with

$$
B(h, c ; r)=\sum_{\varepsilon, \varepsilon^{\prime}} B^{\varepsilon \varepsilon^{\prime}}(h, c ; r),
$$

$$
\begin{aligned}
B^{\varepsilon e^{\prime}}(h, c ; r):= & \frac{1}{D_{1}} \sum_{d(r)}^{*} e\left(\frac{-d h}{r}\right) \sum_{r_{1}, r_{2}, x\left(D_{1}\right)}^{*} \chi_{D_{1}}\left(r_{1}\right) \bar{\chi}_{D_{1}}\left(r_{2}\right) e\left(\frac{r_{1} r_{2} x}{D_{1}}\right) I^{\varepsilon}\left(d, r_{1}, r_{2}\right) I I^{\varepsilon^{\prime}}(d, x) \\
= & \frac{\chi_{D_{2}}^{\varepsilon}\left(\bar{D}_{1} r^{\prime}\right) \chi^{\varepsilon^{\prime}}\left(\frac{a e r^{\prime}}{\left(a e, r^{\prime}\right)^{2}}\right) G\left(\bar{\chi}_{D_{2}}^{\varepsilon}\right) G\left(\bar{\chi}^{\varepsilon^{\prime}}\right) L\left(\chi^{2 \varepsilon^{\prime}}, 1\right)}{r^{2} /\left(a e, r^{\prime}\right)} \frac{1}{\varphi\left(D_{1}\right)} \times \\
& \times \sum_{\psi\left(D_{1}\right)} \bar{\psi}\left(-D_{2}\right) \operatorname{Res}_{s=0} D^{s} L\left(\psi \chi_{D_{2}}^{2 \varepsilon}, 1+s\right) \times \\
& \times \sum_{\substack{d(r) \\
x\left(D_{1}\right)}}^{*} e\left(-\frac{d h}{r}\right)\left(\psi \chi_{D_{2}}^{\varepsilon}\right)(d) \chi^{\varepsilon^{\prime}}\left(d-D_{2} r^{\prime} \bar{x}\right) \times \\
& \times \sum_{r_{1}, r_{2}\left(D_{1}\right)} \chi_{D_{1}}\left(r_{1}\right) \bar{\chi}_{D_{1}}\left(r_{2}\right) \bar{\psi}(r(\varepsilon)) e\left(\frac{r_{1} r_{2}\left(x-r^{\prime} D_{2} \bar{d}\right)}{D_{1}}\right)
\end{aligned}
$$

The $r_{1}$ and $r_{2}$ sums give

$$
\begin{aligned}
& \sum_{r_{1}, r_{2}\left(D_{1}\right)} \chi_{D_{1}}\left(r_{1}\right) \bar{\chi}_{D_{1}}\left(r_{2}\right) \bar{\psi}(r(\varepsilon)) e\left(\frac{r_{1} r_{2}\left(x-r^{\prime} D_{2} \bar{d}\right)}{D_{1}}\right) \\
& \quad=\delta_{\psi=\chi_{D_{1}}^{2 \varepsilon}} \varphi\left(D_{1}\right) G\left(\bar{\chi}_{D_{1}}^{\varepsilon}\right) \chi_{D_{1}}^{\varepsilon}\left(x-r^{\prime} D_{2} \bar{d}\right),
\end{aligned}
$$

So

$$
\begin{aligned}
B^{\varepsilon \varepsilon^{\prime}}(h, c ; r)= & \frac{\chi_{D_{2}}^{\varepsilon}\left(r^{\prime}\right) \bar{\chi}_{D_{1}}^{\varepsilon}\left(D_{2}\right) \chi^{\varepsilon^{\prime}}\left(\frac{a e r^{\prime}}{\left(a e, r^{\prime}\right)^{2}}\right) G\left(\bar{\chi}^{\varepsilon}\right) G\left(\bar{\chi}^{\varepsilon^{\prime}}\right) L\left(\chi^{2 \varepsilon}, 1\right) L\left(\chi^{2 \varepsilon^{\prime}}, 1\right)}{r^{2} /\left(a e, r^{\prime}\right)} \times \\
& \times \sum_{\substack{d(r) \\
x\left(D_{1}\right)}}^{*} e\left(-\frac{d h}{r}\right)\left(\chi_{D_{1}}^{\varepsilon} \chi_{D_{2}}^{\varepsilon+\varepsilon^{\prime}}\right)(d) \chi_{D_{1}}^{\varepsilon-\varepsilon^{\prime}}(x) \chi_{D_{1}}^{\varepsilon+\varepsilon^{\prime}}\left(d x-D_{2} r^{\prime}\right) .
\end{aligned}
$$

We next compute the sum over $d$ and $x$. It factors over moduli into the product $S\left(r^{\prime}\right) S\left(D_{1}\right) S\left(D_{2}\right)$ of 3 sums. We let $d=D_{1} D_{2} d^{\prime}+r^{\prime} D_{1} d_{2}+r^{\prime} D_{2} d_{1}$, so the first sum is 


$$
S\left(r^{\prime}\right)=\sum_{d^{\prime}\left(r^{\prime}\right)}^{*} e\left(-\frac{d^{\prime} h}{r^{\prime}}\right)=r\left(h ; r^{\prime}\right)
$$

and the third sum is

$$
S\left(D_{2}\right)=\chi^{\varepsilon+\varepsilon^{\prime}}\left(r^{\prime}\right) \sum_{d\left(D_{2}\right)}^{*} e\left(-\frac{d_{2} h}{D_{2}}\right) \chi_{D_{2}}^{\varepsilon+\varepsilon^{\prime}}\left(d_{2} D_{1}\right)=\chi_{D_{2}}^{\varepsilon+\varepsilon^{\prime}}\left(D_{1} r^{\prime}\right) G\left(\chi_{D_{2}}^{\varepsilon+\varepsilon^{\prime}}, h ; D_{2}\right) .
$$

The second sum is more complicated:

$$
S\left(D_{1}\right)=\sum_{d_{1}, x\left(D_{1}\right)}^{*} e\left(-\frac{d_{1} h}{D_{1}}\right) \chi_{D_{1}}^{\varepsilon}\left(d_{1} r^{\prime} D_{2}\right) \chi_{D_{1}}^{-\varepsilon^{\prime}}(x) \chi_{D_{1}}^{\varepsilon+\varepsilon^{\prime}}\left(x d_{1} r^{\prime} D_{2}-r^{\prime} D_{2}\right)
$$

Making the variable change $x^{\prime}=x d_{1}$ lets us factor this into a Jacobi sum over $x$ and a Gauss sum over $d_{1}$, giving

$$
S\left(D_{1}\right)=\chi_{D_{1}}^{2 \varepsilon+\varepsilon^{\prime}}\left(-r^{\prime} D_{2}\right) G\left(\chi_{D_{1}}^{\varepsilon+\varepsilon^{\prime}}, h ; D_{1}\right) J\left(\chi_{D_{1}}^{-\varepsilon^{\prime}}, \chi_{D_{1}}^{\varepsilon+\varepsilon^{\prime}}\right)
$$

Combining the various sums thus yields

$$
\begin{aligned}
B^{\varepsilon \varepsilon^{\prime}}(h, c ; r) & \\
= & \frac{\chi_{D_{2}}^{\varepsilon}\left(r^{\prime}\right) \bar{\chi}_{D_{1}}^{\varepsilon}\left(D_{2}\right) \chi^{\varepsilon^{\prime}}\left(\frac{a e r^{\prime}}{\left(a e, r^{\prime}\right)^{2}}\right) G\left(\bar{\chi}^{\varepsilon}\right) G\left(\bar{\chi}^{\varepsilon^{\prime}}\right) L\left(\chi^{2 \varepsilon}, 1\right) L\left(\chi^{2 \varepsilon^{\prime}}, 1\right)}{r^{2} /\left(a e, r^{\prime}\right)} \times \\
& \times r\left(h ; r^{\prime}\right) \chi_{D_{2}}^{\varepsilon+\varepsilon^{\prime}}\left(D_{1} r^{\prime}\right) G\left(\chi_{D_{2}}^{\varepsilon+\varepsilon^{\prime}}, h ; D_{2}\right) \chi_{D_{1}}^{2 \varepsilon+\varepsilon^{\prime}}\left(D_{2} r^{\prime}\right) G\left(\chi_{D_{1}}^{\varepsilon+\varepsilon^{\prime}}, h ; D_{1}\right) J\left(\chi_{D_{1}}^{-\varepsilon^{\prime}}, \chi_{D_{1}}^{\varepsilon+\varepsilon^{\prime}}\right) . \\
= & \frac{\chi^{2\left(\varepsilon+\varepsilon^{\prime}\right)}\left(r^{\prime}\right) \chi^{\varepsilon^{\prime}}\left(\frac{a e}{\left(a e, r^{\prime}\right)^{2}}\right) G\left(\bar{\chi}^{\varepsilon}\right) G\left(\bar{\chi}^{\varepsilon^{\prime}}\right) L\left(\chi^{2 \varepsilon}, 1\right) L\left(\chi^{2 \varepsilon^{\prime}}, 1\right) r\left(h ; r^{\prime}\right)}{r^{2} /\left(a e, r^{\prime}\right)} \times \\
& \times G\left(\chi^{\varepsilon+\varepsilon^{\prime}}, h ; D\right) J\left(\chi_{D_{1}^{-\varepsilon^{\prime}},}^{-\varepsilon}, \chi_{D_{1}}^{\varepsilon+\varepsilon^{\prime}}\right) .
\end{aligned}
$$

The two cases $\varepsilon= \pm \varepsilon^{\prime}$ contribute in slightly different ways. If $\varepsilon=\varepsilon^{\prime}$, then

$$
\begin{aligned}
B^{\varepsilon \varepsilon}(h, c ; r)= & J\left(\chi_{D_{1}}^{-\varepsilon}, \chi_{D_{1}}^{\varepsilon+\varepsilon^{\prime}}\right) \chi^{4 \varepsilon}\left(r^{\prime}\right) \chi^{\varepsilon}\left(\frac{a e}{\left(a e, r^{\prime}\right)^{2}}\right) G\left(\bar{\chi}^{\varepsilon}\right)^{2} G\left(\chi^{2 \varepsilon}, h ; D\right) \times \\
& \times L\left(\chi^{2 \varepsilon}, 1\right)^{2} \frac{r\left(h ; r^{\prime}\right)}{r^{2} /\left(a e, r^{\prime}\right)} .
\end{aligned}
$$

However, if $\varepsilon=-\varepsilon^{\prime}$, then $J\left(\chi_{D_{1}}^{-\varepsilon}, \chi_{D_{1}}^{\varepsilon+\varepsilon^{\prime}}\right)=\mu\left(D_{1}\right)$, so

$$
B^{\varepsilon-\varepsilon}(h, c ; r)=\mu\left(D_{1}\right) \bar{\chi}^{\varepsilon}\left(\frac{a e}{\left(a e, r^{\prime}\right)^{2}}\right)\left|L\left(\chi^{2}, 1\right)\right|^{2} \frac{r(h ; r)}{r r^{\prime} /\left(a e, r^{\prime}\right)} .
$$




\subsection{THE MAIN OFF-OFF-DIAGONAL TERM}

Since $A(h, c ; r)$ vanishes,

$$
\sum_{m_{1}, m_{2}, n} \sum \cdots=\widehat{E}^{v}(1,1) \chi(v) B(h, c ; r)+E r r
$$

where Err is admissible and $v \in\{ \pm 1\}$. Following [DFI1] p. 215, we have

$$
\begin{aligned}
\widehat{E}^{v}(1,1) & =\iint_{0}^{\infty} \hat{F}^{v}(x, y) \Delta_{r}\left(x+v D_{3}^{2} a e y-h\right) \mathrm{d} x \mathrm{~d} y \\
& =\int_{0}^{\infty} \hat{F}^{v}\left(h-v D_{3}^{2} a e y, y\right) \mathrm{d} y+E r r
\end{aligned}
$$

since $\Delta_{r}$ approximate well the Dirac distribution. Hence

$$
\sum_{m_{1}, m_{2}, n} \sum_{n} \cdots(v) B(h, c ; r) \int_{0}^{\infty} \hat{F}^{v}\left(h-v D_{3}^{2} a e y, y\right) \mathrm{d} y+E r r,
$$

where in both equations Err is an admissible error term. Collecting all the terms (ie., summing over $M, N$ ), we find that the main off-off diagonal term is given by

$$
\begin{aligned}
M^{O O D}(\ell)=\chi(-1) \sum_{d e=\ell} & \frac{1}{d^{1 / 2}} \sum_{a b=d} \frac{\mu(a)}{a^{1 / 2}} \tau_{\chi}(b) \times \\
& \times \sum_{\substack{c \equiv 0(q D) \\
c \leqslant q^{A}}} \frac{1}{c^{2}} \sum_{\substack{r=0(D)=\\
(D, r / D)=1}} \sum_{h \neq 0} r^{m}\left(h ; c^{\prime}\right) B(h, c ; r)\left[V^{+}(h)+V^{-}(h)\right]
\end{aligned}
$$

with $A=100$, say, and

$$
\begin{aligned}
V^{ \pm}(h)= & -2 \pi \iint_{0}^{\infty} \delta_{h \mp a e y>0} K^{ \pm}\left(4 \pi \frac{\sqrt{x(h \mp a e y)}}{c}\right) J_{1}\left(4 \pi \frac{\sqrt{x(a e y)}}{c}\right) \times \\
& \times \eta(x) \eta(y) D(x, y) \mathrm{d} x \mathrm{~d} y,
\end{aligned}
$$

where $D$ is defined by (7.4). Since $r^{m}\left(h ; c^{\prime}\right) B(h, c ; r)$ is even in $h$, we can replace $h$ by its absolute value, as in [KMV2], with the effect of replacing $\left[V^{+}(h)+V^{-}(h)\right]$ by (compare [KMV2] (35))

$$
\begin{aligned}
& \frac{h^{1 / 2}}{(a e)^{1 / 2}} \iint(x y)^{-1 / 2} W\left(\frac{x}{g D^{2}}\right) W\left(\frac{d h y}{e q D^{2}}\right) \eta(x) \eta\left(\frac{h y}{a e}\right) J_{1}\left(4 \pi \frac{\sqrt{h x y}}{c}\right) \times \\
& \times\left[-2 \pi Y_{0}\left(4 \pi \frac{\sqrt{h x(1+y)}}{c}\right)-2 \pi \delta_{y>1} Y_{0}\left(4 \pi \frac{\sqrt{h x(y-1)}}{c}\right)+\right. \\
& \left.\quad+4 \delta_{y<1} K_{0}\left(4 \pi \frac{\sqrt{h x(1-y)}}{c}\right)\right] \mathrm{d} x \mathrm{~d} y .
\end{aligned}
$$

One can show, exactly as in [KMV2] 4.1, that we may, up to an admissible error term, drop the constraint $c \leqslant q^{A}$ and replace $\eta(x)$ and $\eta\left(\frac{h y}{a e}\right)$ by 1 . Following 
[KMV2] 4.1-4.3, but with slightly simpler calculations because there are no logarithms, we find that (8.13) equals

$$
\begin{aligned}
& \frac{16(a e)^{-1 / 2}}{(2 \pi \mathrm{i})^{2}} \int_{(1.7)} \int_{(0.6)} \zeta^{(q D)}(1+2 s) \zeta^{(q D)}(1+2 t)\left(\frac{e}{d}\right)^{t} c^{1-2 s} h^{s-t} \frac{\left(q D^{2}\right)^{s+t}}{\left(4 \pi^{2}\right)^{t}} \times \\
& \quad \times G(s) G(t) \Gamma(1+s) \Gamma(1-s) \Gamma(1+t) \Gamma(1-t) \times \\
& \quad \times \cos \left(\pi \frac{t+s}{2}\right) \Gamma(t+s) \cos \left(\pi \frac{t-s}{2}\right) \Gamma(t-s) \frac{\mathrm{d} s \mathrm{~d} t}{s t} .
\end{aligned}
$$

Plugging this into (8.12) we obtain, up to an admissible error term,

where

$$
\begin{aligned}
M^{O O D}(\ell)= & \frac{1}{\ell^{1 / 2}} \frac{16}{(2 \pi \mathrm{i})^{2}} \int_{(1.7)} \int_{(0.6)} \zeta^{(q D)}(1+2 s) \zeta^{(q D)}(1+2 t) L(\ell, s, t) \frac{\left(q D^{2}\right)^{s+t}}{\left(4 \pi^{2}\right)^{t}} \times \\
& \times G(s) G(t) \Gamma(1+s) \Gamma(1-s) \Gamma(1+t) \Gamma(1-t) \times \\
& \times \cos \left(\pi \frac{t+s}{2}\right) \Gamma(t+s) \cos \left(\pi \frac{t-s}{2}\right) \Gamma(t-s) \frac{\mathrm{d} s \mathrm{~d} t}{s t}
\end{aligned}
$$

$$
\begin{aligned}
L(\ell, s, t)= & \chi(-1) \sum_{a b e=\ell} \frac{\mu(a)}{a} \tau_{\chi}(b)\left(\frac{e}{a b}\right)^{t} \sum_{c \equiv 0(q D)} \frac{1}{c^{1+2 s}} \times \\
& \times \sum_{\substack{r=0(D) \\
(D, r)=1}} \sum_{h \geqslant 1} h^{s-t} r^{m}\left(h ; c^{\prime}\right) B(h, c ; r)
\end{aligned}
$$

and $B(h, r ; c)$ is given by $(8.9)$ and $(8.10)$. This concludes the proof of Proposition 7.1.

\section{Computation of $M^{O O D}(\ell)$}

We next need to evaluate $M^{O O D}(\ell)$. The largest remaining step is to compute the formal $L$ series $L(\ell, s, t)$ and its contribution to the off-off-diagonal term. Up to the symmetry $\chi \rightarrow \bar{\chi}$ it is enough to compute $L^{++}(\ell, s, t)$ and $L^{-+}(\ell, s, t)$, defined as in (8.15) but with $B^{++}(h, c ; r)$ and $B^{-+}(h, c ; r)$ instead of $B(h, c ; r)$.

\subsection{COMPUTATION OF $L^{++}(\ell, s, t)$}

We want to factor $L^{++}(\ell, s, t)$ over relatively prime moduli as much as possible. We set

$$
\begin{aligned}
& h=h^{\prime} h^{\prime \prime}, \quad\left(h^{\prime}, D\right)=1, \quad h^{\prime \prime} \mid D^{\infty} ; \\
& c=c^{\prime} D_{1}=c^{\prime \prime} D_{1} D_{2}^{2} D^{\prime}, \quad D^{\prime} \mid D_{2}^{\infty}, \quad\left(c^{\prime \prime}, D\right)=1 ; \\
& r=r^{\prime} D, \quad\left(r^{\prime}, D\right)=1,
\end{aligned}
$$

so that

$$
\begin{aligned}
& r^{m}\left(h ; c^{\prime}\right)=r^{m}\left(h^{\prime} h^{\prime \prime} ; c^{\prime \prime} D_{2}^{2} D^{\prime}\right)=r^{m}\left(h^{\prime} ; c^{\prime \prime}\right) r\left(h^{\prime \prime} ; D_{2}^{2} D^{\prime}\right) \\
& B^{++}(h, c ; r)= \\
& \chi\left(\frac{a e\left(r^{\prime}\right)^{4}}{\left(a e, r^{\prime}\right)^{2}}\right) \bar{\chi}^{2}\left(h^{\prime}\right)\left(a e, r^{\prime}\right) \frac{r\left(h^{\prime} ; r^{\prime}\right)}{\left(r^{\prime}\right)^{2}} J\left(\bar{\chi}_{D_{1}}, \chi_{D_{1}}^{2}\right) \times \\
& \times G\left(\chi^{2}, h^{\prime \prime} ; D\right) \frac{G(\bar{\chi})^{2} L\left(\chi^{2}, 1\right)^{2}}{D^{2}} .
\end{aligned}
$$


Hence we have

$$
\begin{aligned}
L^{++}(\ell, s, t)= & \frac{G(\bar{\chi})^{2} L\left(\chi^{2}, 1\right)^{2}}{D^{2}} \times \\
& \times\left(\chi(-1) \sum_{\substack{D_{1} D_{2}=D \\
D^{\prime} \mid D_{2}^{\infty}}} \frac{J\left(\bar{\chi}_{D_{1}}, \chi_{D_{1}}^{2}\right)}{\left(D_{1} D_{2}^{2} D^{\prime}\right)^{1+2 s}} \sum_{h \mid D^{\infty}} h^{s-t} r\left(h ; D_{2}^{2} D^{\prime}\right) G\left(\chi^{2}, h ; D\right)\right) \times \\
& \times\left(\sum_{a b e=\ell} \frac{\mu(a)}{a} \tau_{\chi}(b)\left(\frac{e}{a b}\right)^{t} \chi(a e) \sum_{c \equiv 0(q)}^{\prime} \frac{1}{c^{1+2 s}} \sum_{r}^{\prime} \chi^{4}(r) \frac{\bar{\chi}^{2}((a e, r))(a e, r)}{r^{2}} \times\right. \\
& \left.\times \sum_{h \geqslant 1}^{\prime} \bar{\chi}^{2}(h) r(h ; r) r^{m}(h ; c)^{t-s}\right) \\
:= & \frac{G(\bar{\chi})^{2} L\left(\chi^{2}, 1\right)^{2}}{D^{2}} L_{D}^{++}(\ell, s, t) L^{(D),++}(\ell, s, t)
\end{aligned}
$$

where the $\sum^{\prime}$ means sum over integers coprime with $D$.

\subsubsection{Computation of $L^{(D),++}(\ell, s, t)$}

We follow [KMV2], (43) through (45): using (8.2) we have (setting $A=a e$ )

$$
\begin{aligned}
& L^{(D),++}(\ell, s, t) \\
& =\frac{1}{\ell^{t}} \sum_{A b=\ell} \tau_{\chi}(b) A^{2 t} \zeta_{A}(1+2 t)^{-1} \chi(A) \sum_{(g, q)=1}^{\prime} \frac{\mu(g)}{g^{1+2 s}} \times \\
& \quad \times \sum_{c \equiv 0(q)}^{\prime} \frac{1}{c^{2 s}} \sum_{v} \frac{\mu(v) \chi^{4}(v)}{v^{2}} \sum_{w}^{\prime} \chi^{4}(w) \frac{\bar{\chi}^{2}((A, v w))(A, v w)}{w} \sum_{c, w \mid h} \frac{\bar{\chi}^{2}(h)}{h^{t-s}} .
\end{aligned}
$$

The $g$ sum is $\zeta^{(q D)}(1+2 s)^{-1}$. We separate variables using the identity

$$
\sum_{c, w} f(c, w) \sum_{c, w \mid h} g(h)=\sum_{u, x} \mu(u) \sum_{c, w} f(u x c, u x w) \sum_{h} g\left(c w u^{2} x h\right) .
$$

We factor the resulting $c$ and $h$ sums to obtain (compare with [KMV2] (43))

$$
\begin{aligned}
L^{D,++} & (\ell, s, t) \\
= & \frac{\bar{\chi}^{2}(q) L\left(\bar{\chi}^{2}, t+s\right) L\left(\bar{\chi}^{2}, t-s\right)}{q^{s+t \zeta^{(q D)}}(1+2 s)} \frac{1}{\ell^{t}} \sum_{A b=\ell} \tau_{\chi}(b) A^{2 t} \zeta_{A}(1+2 t)^{-1} \chi(A) \times \\
& \times \sum_{v} \frac{\mu(v) \chi^{4}(v)}{v^{2}} \sum_{x} \frac{\chi^{2}(x)}{x^{1+t+s}} \sum_{(u, q)=1}^{\prime} \frac{\mu(u)}{u^{1+2 t}} \sum_{w} \chi^{2}(w) \frac{\bar{\chi}^{2}((A, u v w x))(A, u v w x)}{w^{1+t-s}} .
\end{aligned}
$$


Next we factor $u=u_{1} u_{2}$ with $u_{1} \mid A,\left(u_{2}, A\right)=1$, replace $A$ by $u_{1} A$, sum over $u_{2}$, and write $u_{1} b=B$ to obtain

$$
\begin{aligned}
L^{D,++}(\ell, s, t) & \\
= & \frac{\chi(\ell) \bar{\chi}^{2}(q) L\left(\bar{\chi}^{2}, t+s\right) L\left(\bar{\chi}^{2}, t-s\right)}{q^{s+t} \zeta^{(q D)}(1+2 s) \zeta^{(q D)}(1+2 t)} \times \\
& \times \frac{1}{\ell^{t}} \sum_{A B=\ell} A^{2 t} \sum_{v} \frac{\mu(v) \chi^{4}(v)}{v^{2}} \sum_{x} \frac{\chi^{2}(x)}{x^{1+t+s}} \sum_{w} \chi^{2}(w) \frac{\bar{\chi}^{2}((A, v w x))(A, v w x)}{w^{1+t-s}} .
\end{aligned}
$$

We factor $v=v_{1} v_{2}$ as we did for $u$, replacing $A$ by $A v_{2}$, so the $v_{1}$ sum equals $L_{A v_{2}}\left(\chi^{4}, 2\right) L\left(\chi^{4}, 2\right)^{-1}$. We combine the product $x w$ into a single variable $w$, and use the relation

$$
\sum_{A, w} \bar{\chi}^{2}((A, w))(A, w) f(A, w)=\sum_{E} E \bar{\chi}^{2}(E) L_{E}\left(\chi^{2}, 1\right)^{-1} \sum_{A, w} f(E A, E w),
$$

together with

$$
\sum_{E, w} \frac{\sigma_{2 s}(E w)}{(E w)^{s}} g(E, w)=\sum_{a} \mu(a) \sum_{E, w} \frac{\sigma_{2 s}(E)}{E^{s}} \frac{\sigma_{2 s}(w)}{w^{s}} g(a E, a w)
$$

to end up with

$$
\begin{aligned}
& L^{(D),++}(\ell, s, t) \\
& =\frac{\bar{\chi}^{2}(q) L\left(\chi^{2}, 1+t+s\right) L\left(\chi^{2}, 1+t-s\right) L\left(\bar{\chi}^{2}, t+s\right) L\left(\bar{\chi}^{2}, t-s\right)}{q^{s+t} L\left(\chi^{4}, 2\right) \zeta^{(q D)}(1+2 s) \zeta^{(q D)}(1+2 t)} \times \\
& \quad \times \frac{\chi(\ell)}{\ell^{t}} \sum_{a A E \mid \ell} A^{2 t} L_{A}\left(\chi^{2}, 1\right)^{-1} E^{t} \frac{\sigma_{2 s}(E)}{E^{s}} \frac{\mu(a) \chi^{2}(a)}{a} L_{a E}\left(\chi^{2}, 1\right)^{-1} L_{a A E}\left(\chi^{4}, 2\right) .
\end{aligned}
$$

\subsubsection{Computation of $L_{D}^{++}(\ell, s, t)$}

Since $\chi^{2}$ is primitive, $r\left(h ; D_{2}^{2} D^{\prime}\right) G\left(\chi^{2}, h ; D\right)$ is zero unless $h=1$ and $D_{2}^{2} D^{\prime}=1$, so that $D_{1}=D$. This simplifies calculations considerably, leaving us with

$$
L_{D}^{++}(\ell, s, t)=\chi(-1) \frac{J\left(\bar{\chi}_{D}, \chi_{D}^{2}\right) G\left(\chi^{2}\right)}{D^{1+2 s}}=D^{-2 s} \frac{G\left(\chi^{2}\right)^{2}}{G(\chi)^{2}} .
$$

\subsubsection{Computation of the off-off diagonal term I}

Using (8.14) (9.1), (9.2), and (9.3) we see that the contribution of $L^{++}(\ell, s, t)$ to $M^{O O D}(\ell)$ is

$$
\begin{aligned}
M^{O O D,++}(\ell)= & \frac{\bar{\chi}^{2}(q)}{\ell^{1 / 2}} \frac{G\left(\chi^{2}\right)^{2}}{G(\chi)^{4}} \frac{L\left(\chi^{2}, 1\right)^{2}}{L\left(\chi^{4}, 2\right)} \times \\
& \times \frac{16}{(2 \pi \mathrm{i})^{2}} \int_{(1.7)} \int_{(0.6)} L\left(\chi^{2}, 1+t+s\right) L\left(\chi^{2}, 1+t-s\right) L\left(\bar{\chi}^{2}, t+s\right) \times
\end{aligned}
$$




$$
\begin{aligned}
& \times L\left(\bar{\chi}^{2}, t-s\right) \frac{D^{2 t}}{(2 \pi)^{2 t}} \times G(s) G(t) \Gamma(1+s) \Gamma(1-s) \Gamma(1+t) \Gamma(1-t) \times \\
& \times \cos \left(\pi \frac{t+s}{2}\right) \Gamma(t+s) \cos \left(\pi \frac{t-s}{2}\right) \Gamma(t-s) \times \\
& \times \frac{\chi(\ell)}{\ell^{t}} \sum_{a A E \mid \ell} A^{2 t} L_{A}\left(\chi^{2}, 1\right)^{-1} E^{t} \frac{\sigma_{2 s}(E)}{E^{s}} \frac{\mu(a) \chi^{2}(a)}{a} L_{a E}\left(\chi^{2}, 1\right)^{-1} \times \\
& \times L_{a A E}\left(\chi^{4}, 2\right) \frac{\mathrm{d} s \mathrm{~d} t}{s t} .
\end{aligned}
$$

Since $\chi^{2}$ is primitive and $\chi^{2}(-1)=1, L\left(\bar{\chi}^{2}, s\right)$ satisfies the asymmetric functional equation

$$
2\left(\frac{D}{2 \pi}\right)^{s} \cos (\pi s / 2) \Gamma(s) L\left(\bar{\chi}^{2}, s\right)=G\left(\bar{\chi}^{2}\right) L\left(\bar{\chi}^{2}, 1-s\right) .
$$

Applying this twice we obtain

$$
\begin{aligned}
& M^{O O D,++}(\ell) \\
& =\frac{\bar{\chi}^{2}(q)}{\ell^{1 / 2}} \frac{D^{2}}{G(\chi)^{4}} \frac{L\left(\chi^{2}, 1\right)^{2}}{L\left(\chi^{4}, 2\right)} \times \\
& \quad \times \frac{4}{(2 \pi \mathrm{i})^{2}} \int_{(1.7)} \int_{(0.6)} G(s) G(t) \prod_{ \pm} \Gamma(1 \pm s) \Gamma(1 \pm t) \prod_{ \pm, \pm} L\left(\chi^{2}, 1 \pm t \pm s\right) \times \\
& \quad \times \frac{\chi(\ell)}{\ell^{t}} \sum_{a A E \mid \ell} A^{2 t} L_{A}\left(\chi^{2}, 1\right)^{-1} E^{t} \frac{\sigma_{2 s}(E)}{E^{s}} \frac{\mu(a) \chi^{2}(a)}{a} L_{a E}\left(\chi^{2}, 1\right)^{-1} L_{a A E}\left(\chi^{4}, 2\right) \frac{\mathrm{d} s \mathrm{~d} t}{s t} .
\end{aligned}
$$

Let $\Phi(s, t, \ell)$ be the multiplicative function

$$
\Phi(s, t ; \ell)=\frac{1}{\ell^{t}} \sum_{a A E \mid \ell} A^{2 t} L_{A}\left(\chi^{2}, 1\right)^{-1} E^{t} \frac{\sigma_{2 s}(E)}{E^{s}} \frac{\mu(a) \chi^{2}(a)}{a} L_{a E}\left(\chi^{2}, 1\right)^{-1} L_{a A E}\left(\chi^{4}, 2\right) .
$$

One can check (compare with [KMV2] 4.3.1) that $\Phi(s, t ; \ell)$ is an even function is both $s$ and $t$ : obviously we may assume that $\ell=p^{\alpha}$ for some prime $p \nmid D$. Evenness in $s$ is obvious, but evenness in $t$ requires a case-by-case breakdown of whether $p$ divides each of the variables. More precisely we rewrite

$$
\Phi(s, t ; \ell)=\sum_{a c A E=\ell}\left(\frac{A}{a c}\right)^{t} L_{A}\left(\chi^{2}, 1\right)^{-1} \frac{\sigma_{2 s}(E)}{E^{s}} \frac{\mu(a) \chi^{2}(a)}{a} L_{a E}\left(\chi^{2}, 1\right)^{-1} L_{a A E}\left(\chi^{4}, 2\right)
$$

and we split the sum according to three cases which are even in $s$ and $t$ :

- If $p \mid E$, we have

$$
\sum_{\substack{E \ell^{\prime}=\ell \\ E>1}} \frac{\sigma_{2 s}(E)}{E^{s}} \frac{1}{1+\frac{\chi^{2}(p)}{p}} \sum_{A B=\ell^{\prime}}\left(\frac{A}{B}\right)^{t} L_{A}\left(\chi^{2}, 1\right)^{-1} L_{B}\left(\chi^{2}, 1\right)^{-1} .
$$


- If $E=1$, and either $A=\ell$ or $a c=\ell$, we have

$$
\ell^{t} \frac{1}{1+\frac{\chi^{2}(p)}{p}}+\ell^{-t}\left(1-\frac{\chi^{2}}{p\left(1+\frac{\chi^{2}(p)}{p}\right)}\right)=\frac{\ell^{t}+\ell^{-t}}{1+\frac{\chi^{2}(p)}{p}} .
$$

- If $E=1$, and $p \mid A$ and $p \mid a c$, we have

$$
\begin{aligned}
& \frac{1}{1+\frac{\chi^{2}(p)}{p}} \sum_{A B=\ell / p^{2}}\left(\frac{A}{B}\right)^{t} \sum_{a c=B p} \frac{\mu(a) \chi^{2}(a)}{a} L_{a}\left(\chi^{2}, 1\right)^{-1} \\
& =\frac{1-\frac{\chi^{2}(p)}{p}+\frac{\chi^{4}(p)}{p^{2}}}{1+\frac{\chi^{2}(p)}{p}} \sum_{A B=\ell / p^{2}}\left(\frac{A}{B}\right)^{t} .
\end{aligned}
$$

From (9.4) it follows that the integrand in $M^{O O D,++}(\ell)$ is an odd function of $s$ and $t$. Thus, by shifting the contours in $s$ and $t$, we find that $M^{O O D,++}(\ell)$ equals one-fourth of the residue at $s=t=0$ of the integrand (since the polynomial $G(s)$ vanishes at $s= \pm 1$, the $\Gamma$ factors contribute no poles). The same holds for $L^{--}(s, t ; \ell)$ with $\chi$ exchanged for $\bar{\chi}$, thus

$$
M^{O O D, \varepsilon \varepsilon}(\ell)=\bar{\chi}^{2 \varepsilon}(q) \frac{D^{2}}{G\left(\chi^{\varepsilon}\right)^{4}} \frac{L\left(\chi^{2 \varepsilon}, 1\right)^{6}}{L\left(\chi^{4 \varepsilon}, 2\right)} \frac{\chi^{\varepsilon}(\ell)}{\ell^{1 / 2}} \Phi^{\varepsilon}(\ell)
$$

with

$$
\Phi^{\varepsilon}(\ell)=\sum_{a A E \mid \ell} L_{A}\left(\chi^{2 \varepsilon}, 1\right)^{-1} \tau(E) \frac{\mu(a) \chi^{2 \varepsilon}(a)}{a} L_{a E}\left(\chi^{2 \varepsilon}, 1\right)^{-1} L_{a A E}\left(\chi^{4 \varepsilon}, 2\right) .
$$

\subsection{COMPUTATION OF $L^{-+}(\ell, s, t)$}

Next we compute

$$
\begin{aligned}
& L^{-+}(\ell, s, t) \\
& =\chi(-1)\left|L\left(\chi^{2}, 1\right)\right|^{2} \sum_{a b e=\ell} \frac{\mu(a)}{a} \tau_{\chi}(b)\left(\frac{e}{a b}\right)^{t} \sum_{c \equiv 0(q D)} \frac{1}{c^{1+2 s}} \times \\
& \quad \times \sum_{\left(D, r^{\prime}\right)=1} \sum_{h \geqslant 1} h^{s-t} r^{m}\left(h ; c^{\prime}\right) \mu\left(D_{1}\right) \chi(a e) \chi\left(\left(a e, r^{\prime}\right)\right)^{2} \frac{r\left(h ; r^{\prime} D\right)\left(a e, r^{\prime}\right)}{D\left(r^{\prime}\right)^{2}} .
\end{aligned}
$$

The calculation is again done by factoring over primes and grouping those primes'into three disjoint sets: those dividing $D$, those dividing $\ell$, and those dividing'neither. The last set requires exactly the same computations as were done in [KMV2], except slightly simpler since there are no logarithms involved, and we wind up with

$$
\begin{aligned}
q^{-s-t} \zeta^{(q \ell D)}(1+2 s)^{-1} \zeta^{(q \ell D)}(1+2 t)^{-1} \zeta^{(\ell D)}(2)^{-1} \\
\quad \times \prod_{ \pm} \zeta^{(\ell D)}(1 \pm s+t) \zeta_{\ell D}(t \pm s) .
\end{aligned}
$$


The product over primes dividing $\ell$ is similar both to the calculations of the previous'section and to those of [KMV2]. Introducing the same variables to account for the divisibility conditions on $h$ and the various common divisors, we wind up with

$$
\begin{aligned}
& \chi(\ell) \prod_{p \mid \ell} \frac{\left(1-p^{-1-2 s}\right)\left(1-p^{-1-2 t}\right)\left(1-p^{-2}\right)}{\left(1-p^{-1-t-s}\right)\left(1-p^{-1-t+s}\right)\left(1-p^{-t-s}\right)\left(1-p^{-t+s}\right)} \times \\
& \quad \times \frac{1}{\ell^{t}} \sum_{a E A \mid \ell} A^{2 t} L_{A}\left(\bar{\chi}^{2}, 1\right)^{-1} E^{t} \frac{\sigma_{2 s}(E) \bar{\chi}(E)^{2}}{E^{s}} \frac{\mu(a) \bar{\chi}(a)^{2}}{a} L_{a E}\left(\chi^{2}, 1\right)^{-1} \zeta_{a A E}(2) .
\end{aligned}
$$

Let $\Psi(s, t ; \ell)$ be the multiplicative function of $\ell$ given on the second line of (9.8). Once again, one can check that $\Psi(s, t ; \ell)$ is even in both $s$ and $t$. From (9.7) and (9.8), the contribution from all primes not dividing $D$ is now

$$
\begin{aligned}
& \frac{\chi(\ell)}{q^{s+t}} \zeta^{(q D)}(1+2 s)^{-1} \zeta^{(q D)}(1+2 t)^{-1} \zeta^{(D)}(2)^{-1} \times \\
& \quad \times \prod_{ \pm} \zeta^{(D)}(1 \pm s+t) \zeta^{(D)}(t \pm s) \Psi(s, t ; \ell) .
\end{aligned}
$$

It thus remains to calculate the contribution from primes dividing $D$. Since $(D, \ell)=1$, several things simplify, and we are left with

$$
L_{D}^{-+}(s, t)=\sum_{D|c| D^{\infty}} \frac{\mu\left(D_{1}\right)}{c^{1+2 s}} \sum_{h \mid D^{\infty}} h^{s-t} r\left(h ; c^{\prime}\right) r(h ; D) / D,
$$

where $c^{\prime}=c / D, D_{1} D_{2}=D$ with $D_{2}=\left(D, c^{\prime}\right)$. Again, this sum factors over primes. The important distinction is whether a given prime divides $c$ exactly once (in which case it divides $D_{1}$ ), or more than once (in which case it divides $D_{2}$ ). The first case yields

$$
p^{-2-2 s} \mu(p) \sum_{l \geqslant 0} p^{l(s-t)} r\left(p^{l} ; p\right)=-p^{-2-2 s} \frac{p^{1+s-t}-1}{1-p^{s-t}}=-p^{-1-s-t} \frac{1-p^{-1-s+t}}{1-p^{s-t}},
$$

while the second yields

$$
\sum_{k \geqslant 2} p^{-k(1+2 s)} \frac{p-1}{p} \sum_{l \geqslant k-1} p^{l(s-t)} r\left(p^{l} ; p^{k}\right)=\left(1-p^{-1}\right) \frac{1-p^{-1-s+t}}{1-p^{s-t}} \frac{p^{-2 s-2 t}}{1-p^{-s-t}} .
$$

Combining the terms, we have

$$
p^{-2 s-2 t} \frac{\left(1-p^{-1-s+t}\right)\left(1-p^{-1+s+t}\right)}{\left(1-p^{s-t}\right)\left(1-p^{-s-t}\right)} .
$$


From this last equation and (9.9) we obtain that the full product is

$$
\begin{aligned}
L^{-+}(\ell, s, t)= & \frac{\chi(\ell)}{\left(q D^{2}\right)^{s+t}} \frac{\chi(-1)\left|L\left(\chi^{2}, 1\right)\right|^{2}}{\zeta^{(q D)}(1+2 s) \zeta^{(q D)}(1+2 t)} \zeta^{(D)}(2)^{-1} \times \\
& \times \prod_{ \pm} \frac{\zeta^{(D)}(1 \pm s+t) \zeta(t \pm s)}{\zeta^{(D)}(1 \pm s-t)} \Psi(s, t ; \ell) .
\end{aligned}
$$

Again, $L^{+-}$is the same except with $\chi$ and $\bar{\chi}$ switched.

\subsubsection{Computation of the Off-Off-Diagonal Term II}

Putting this back in (8.14) and using the functional equation

$$
\frac{\Gamma(t \pm s) \zeta(t \pm s)}{(2 \pi)^{t \pm s}}=\frac{\zeta(1-(t \pm s))}{2 \cos (\pi(t \pm s) / 2)}
$$

we find that the contribution of $B^{-+}$is

$$
\begin{aligned}
M^{O O D-+}(\ell)= & 4 \frac{\chi(-1)\left|L\left(\chi^{2}, 1\right)\right|^{2}}{\zeta^{(D)}(2)(2 \pi \mathrm{i})^{2}} \int_{(1.7)} \int_{(0.6)} \frac{\chi(\ell)}{\ell^{1 / 2}} \Psi(s, t ; \ell) \times \\
& \times G(s) G(t) \prod_{ \pm} \Gamma(1 \pm s) \Gamma(1 \pm t) \prod_{ \pm, \pm} \zeta^{(D)}(1 \pm s \pm t) \frac{\mathrm{d} s \mathrm{~d} t}{s t}
\end{aligned}
$$

and since the whole integrand is now evidently odd in $s$ and $t$ we have

$$
\begin{aligned}
M^{O O D-+}(\ell)= & \operatorname{Res}_{s=t=0} \frac{\chi(-1)\left|L\left(\chi^{2}, 1\right)\right|^{2}}{\zeta^{(D)}(2)} \frac{\chi(\ell)}{\ell^{1 / 2}} \Psi(s, t ; \ell) \times \\
& \times \frac{G(s) G(t)}{s t} \prod_{ \pm} \Gamma(1 \pm s) \Gamma(1 \pm t) \prod_{ \pm, \pm} \zeta^{(D)}(1 \pm s \pm t) .
\end{aligned}
$$

\subsection{COMPUTATION OF THE 'PURE' FOURTH MOMENT}

We now analyze the case $\ell=1$ in greater detail. From Proposition 7.1 we have for all $\varepsilon>0$

$$
M_{4}(1)=M^{D}(1)+M^{O D}(1)+2 \Re e\left(M^{O O D++}(1)+M^{O O D-+}(1)\right)+O_{\varepsilon, D}\left(q^{-1 / 12+\varepsilon}\right) .
$$

where $M^{D}(1)+M^{O D}(1)$ is given by the first term of (7.9), and the other terms are given by (9.5), (9.10). Shifting the $t$-contour in the integral defining $W\left(n / q D^{2}\right)$ to $\Re e t=-1 / 2$ we see that, up to an admissible remainder term, $M^{D}(1)+M^{O D}(1)$ equals

$$
\begin{aligned}
& \operatorname{Res}_{s=t=0} 4 \frac{G(s) G(t) \Gamma^{2}(1+s) \Gamma^{2}(1+t)}{s t} \times \\
& \quad \times\left(\frac{q D^{2}}{\pi^{2}}\right)^{s+t} \zeta^{(D)}(1+2 s) \zeta^{(D)}(1+2 t) \sum_{n \geqslant 1} \frac{\tau_{\chi}(n)^{2}}{n^{1+s+t}} .
\end{aligned}
$$


Since

$$
\sum_{n \geqslant 1} \frac{\tau_{\chi}(n)^{2}}{n^{1+s+t}}=\frac{L\left(\chi^{2}, 1+s+t\right) L\left(\bar{\chi}^{2}, 1+s+t\right)}{\zeta^{(D)}(2+2 s+2 t)} \zeta^{(D)}(1+s+t)^{2},
$$

we see that $M^{D}(1)+M^{O D}(1)$ is a polynomial in $\log \left(q D^{2} / 4 \pi^{2}\right)$ whose highest degree term is given by

$$
\begin{aligned}
4 & \frac{G(1)^{2} \Gamma^{2}(1) \zeta_{D}(1)^{-4}\left|L\left(\chi^{2}, 1\right)\right|^{2}}{\zeta^{(D)}(2)} \operatorname{Res}_{s=t=0} \frac{\left(\frac{q D^{2}}{4 \pi^{2}}\right)^{s+t}}{s t(s+t)^{2} 2 s 2 t} \\
& =\frac{\left|L\left(\chi^{2}, 1\right)\right|^{2}}{12 \zeta(2)} \prod_{p \mid D} \frac{(1-1 / p)^{3}}{(1+1 / p)} \log \left(\frac{q D^{2}}{4 \pi^{2}}\right)^{4} .
\end{aligned}
$$

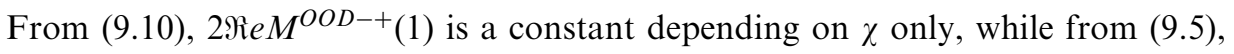

$$
2 \Re e M^{O O D++}(1)=2 \Re e\left(\bar{\chi}^{2}(q) \frac{D^{2}}{G(\chi)^{4}} \frac{L\left(\chi^{2}, 1\right)^{6}}{L\left(\chi^{4}, 2\right)}\right) .
$$

This concludes the proof of Proposition 1.2.

\section{Mollification}

In this section we evaluate the mollified moments, using the asymptotics already calculated. Following the notation of [KMV2], Section 5, we introduce the following conventions:

- For $\boldsymbol{z}=\left(z_{1}, z_{2}, z_{3}, z_{4}\right) \in \mathbf{C}^{4}$ we use $v(s, t, \boldsymbol{z}, \ell)$ for any arithmetic function of the form

$$
v(s, t, \boldsymbol{z}, \ell)=\prod_{p \mid \ell}\left(1+\frac{1}{p} f(s, t, \boldsymbol{z}, p)\right)
$$

with $|f(s, t, \boldsymbol{z}, p)|=O\left(p^{3 / 4}\right)$ uniformly for $s, t, \boldsymbol{z}$ in the domain $s, t, \Re e z_{i} \geqslant-1 / 4$.

- We use $\eta(s, t, z)$ to denote any Euler product of the form

$$
\eta(s, t, \boldsymbol{z})=\prod_{p}\left(1+\frac{1^{2}}{p} f(s, t, \boldsymbol{z}, p)\right)
$$

absolutely convergent and bounded (together with its low partial derivatives) for $z$ in the domain $\Re e z_{i} \geqslant-1 / 4$.

- Our notations are 'generic' in the sense that the exact value of $v(s, t, \boldsymbol{z}, m)$ or $\eta(s, t, z)$ may change from line to line.

The idea is that factors of this nature appear naturally in mollified moments but do not affect the degrees of any poles at the origin, so we can ignore them unless we 
need to compute the exact values of the residues. Their main advantage is that any Euler product that occurs below will be considered as a factor of type $\eta(s, t, z)$ times a product of degree one Euler products. In the event that we would want to know the proportion of non-vanishing precisely, we would want to be very careful with the exact value of such functions (especially at the origin, where we will be evaluating everything), but since the goal of this paper is merely to prove the existence of such a constant, we can afford to be a little bit less precise.

The mollifiers we use will have the following form: for $R>1$ fixed (independent of $\left.\chi_{1}, \chi_{2}, \chi_{3}\right)$ let $P_{R}$ be the product of primes less than $R$. We set

$$
x_{\chi}(\ell)= \begin{cases}0, & \text { if }\left(\ell, P_{R}\right) \neq 1, \\ \chi(\ell) \mu(\ell) P(\log (\ell / L)), & \text { else; }\end{cases}
$$

with $L=q^{\Delta}$ for some fixed small $\Delta$. We take

$$
P_{n}(x)=\frac{1}{2 \pi \mathrm{i}} \int_{(3)} \mathrm{e}^{-x z} \frac{\mathrm{d} z}{z^{n}},
$$

a degree-( $(n-1)$ polynomial on $(-\infty, 0)$ which is zero identically on $[0, \infty)$.

\subsection{THE THIRD MOMENT}

We use Theorem (6.1). Suppose first that all three characters are complex. The leading term of the mollified third moment will come from $P M\left(\chi_{1}, \chi_{2}, \chi_{3}\right)$, the other three $P M$-type terms contributing lower powers of $\log q$. In order to cover all four terms at once, we consider the general expression

$$
P M\left(\chi_{A}, \chi_{B}, \chi_{C}\right)=L\left(1, \chi_{A} \chi_{B}\right) \sum_{d, \ell, n} \frac{x(d \ell) \chi_{A} * \chi_{B}(\ell n) \chi_{C}(d n)}{d \ell n} V\left(\frac{d n}{q^{1 / 2} D_{3}}\right),
$$

where $\chi_{A}, \chi_{B}$, and $\chi_{C}$ are distinct non conjugate characters to be determined later.

Using (10.1) we see that

$$
\begin{array}{rl}
P & M\left(\chi_{A}, \chi_{B}, \chi_{C}\right) \\
= & L\left(1, \chi_{A} \chi_{B}\right) \sum_{\mathrm{d}, \ell, n} \frac{\chi_{A} * \chi_{B}(\ell n) \chi_{C}(d n)}{\mathrm{d} \ell n} \times \\
& \times \sum_{d_{1}, d_{3}} \frac{1}{d_{1} d_{3}} \sum_{\ell_{3} \ell_{4}=d \ell} \sum_{\ell_{1} \ell_{2}=d_{3} \ell_{4}} \chi_{1}\left(d_{1} \ell_{1}\right) \chi_{2}\left(d_{1} \ell_{2}\right) \chi_{3}\left(d_{3} \ell_{3}\right) \mu\left(d_{1} \ell_{1}\right) \mu\left(d_{1} \ell_{2}\right) \mu\left(d_{3} \ell_{3}\right) \times \\
& \times \frac{1}{(2 \pi \mathrm{i})^{4}} \iiint \int \Gamma(1+s)\left(\frac{q^{1 / 2} D_{3}}{d n}\right)^{s} \frac{L^{z_{1}+z_{2}+z_{3}}}{d_{1}^{z_{1}+z_{2}} d_{3}^{z_{3}} \ell_{1}^{z_{1}} \ell_{2}^{z_{2}} \ell_{3}^{z_{3}}} \frac{\mathrm{d} s}{s} \frac{\mathrm{d} z_{1} \mathrm{~d} z_{2} \mathrm{~d} z_{3}}{z_{1}^{n_{1}} z_{2}^{n_{2}} z_{3}^{n_{3}}} .
\end{array}
$$

Since the only important issue is the presence of poles, it is enough to assume that $d_{1} d_{3} d \ell n$ is square-free-all other terms can be collapsed into $\eta$ functions. Thus, given a prime $\mathrm{p}$, it is enough to consider the contributions to first order when it divides each of $d, \ell, n, d_{1}$ and $d_{3}$. Some straightforward analysis gives 


$$
\begin{aligned}
P M & \left(\chi_{A}, \chi_{B}, \chi_{C}\right) \\
= & \frac{L\left(\chi_{A} \chi_{B}, 1\right)}{(2 \pi \mathrm{i})^{4}} \iiint \int \Gamma(1+s) q^{s / 2} D_{3}^{s} \eta\left(s, z_{1}, z_{2}, z_{3}\right) \times \\
& \times L\left(\chi_{A} \chi_{C}, 1+s\right) L\left(\chi_{B} \chi_{C}, 1+s\right) \times \\
& \times \frac{L^{z_{1}+z_{2}+z_{3}} L\left(\chi_{1} \chi_{2}, 1+z_{1}+z_{2}\right) L\left(\chi_{1} \chi_{3}, 1+z_{1}+z_{3}\right) L\left(\chi_{2} \chi_{3}, 1+z_{2}+z_{3}\right)}{\prod_{i=1}^{3} L\left(\chi_{i} \chi_{A}, 1+z_{i}\right) L\left(\chi_{i} \chi_{B}, 1+z_{i}\right) L\left(\chi_{i} \chi_{C}, 1+z_{i}+s\right)} \times \\
& \times \frac{\mathrm{d} s}{s} \frac{\mathrm{d} z_{1} \mathrm{~d} z_{2} \mathrm{~d} z_{3}}{z_{1}^{n_{1}} z_{2}^{n_{2}} z_{3}^{n_{3}}} .
\end{aligned}
$$

for some $\eta\left(s, z_{1}, z_{2}, z_{3}\right)$. Now we evaluate by shifting contours to the left. Since we are assuming $q$ to be much larger than $L$, we can first shift $s$ to $\Re s=-1 / 10$ and each $z_{i}$ to $\Re z_{i}=1 / 10$, and bound the resulting contour integral by $q^{-1 / 20} L^{3 / 10}$, which is small enough to ignore. The only pole we cross in the process is at $s=0$, and it is a simple pole since $\chi_{A}, \chi_{B}$, and $\chi_{C}$ are distinct and non-conjugate. Thus we have, up to negligible error,

$$
\begin{array}{rl}
P & M\left(\chi_{A}, \chi_{B}, \chi_{C}\right) \\
= & \frac{L\left(\chi_{A} \chi_{B}, 1\right) L\left(\chi_{A} \chi_{C}, 1\right) L\left(\chi_{B} \chi_{C}, 1\right)}{(2 \pi \mathrm{i})^{3}} \iiint L^{z_{1}+z_{2}+z_{3}} \eta\left(z_{1}, z_{2}, z_{3}\right) \times \\
& \times \frac{L\left(\chi_{1} \chi_{2}, 1+z_{1}+z_{2}\right) L\left(\chi_{1} \chi_{3}, 1+z_{1}+z_{3}\right) L\left(\chi_{2} \chi_{3}, 1+z_{2}+z_{3}\right)}{\prod_{i=1}^{3} L\left(\chi_{i} \chi_{A}, 1+z_{i}\right) L\left(\chi_{i} \chi_{B}, 1+z_{i}\right) L\left(\chi_{i} \chi_{C}, 1+z_{i}\right)} \frac{\mathrm{d} z_{2} \mathrm{~d} z_{3}}{z_{1}^{n_{1}} z_{2}^{n_{2}} z_{3}^{n_{3}}} .
\end{array}
$$

Now we shift the $z$ contours to the left, one at a time. Were we to assume the Riemann Hypothesis for these $L$-functions, this would be simple, we could shift each to $\Re e z_{i}=-1 / 10$ without hitting any poles. However, as discussed in detail in [KMV2], we don't need the Riemann Hypothesis to bound the resulting contour integrals if we just shift to a contour lying to the right of all the zeros of the arious $L$-functions. Since nonvanishing of these $L$-functions has been proved in small ranges of the critical strip, this is enough (again, see [KMV2] 5.3 for a detailed discussion of how to bound the contour integrals). Thus all we have left is the residues at $z_{1}=z_{2}=z_{3}=0$, and it is here that we need to be more careful about poles. For reasons discussed while analyzing the fourth moment, we will always be taking $n_{i}=4$.

Suppose first that the characters are all complex. If all of the $L$-functions in the denominator come from nontrivial characters, then all the poles are degree 4 , and the leading term goes as $(\log L)^{9}$. However, if (for example) $\chi_{A}=\overline{\chi_{1}}$, the $z_{1}$ pole is only a triple pole, so the lead term goes at most as $(\log L)^{8}$. Thus the largest term in (6.6) when all of the characters are complex is the one coming from $P M\left(\chi_{1}, \chi_{2}, \chi_{3}\right)$, not from any of the conjugates, and that main term is

$$
P M\left(\chi_{1}, \chi_{2}, \chi_{3}\right)=\frac{\eta(0,0,0)}{\prod_{i=1}^{3} L\left(\chi_{i}^{2}, 1\right)}(\log L)^{9} .
$$


It remains to see that $\eta(0,0,0)$ is bounded away from zero for all choice of characters. Had we written $\eta\left(z_{1}, z_{2}, z_{3}\right)$ out explicitly, it would have been an Euler product over primes not dividing $P_{R}$ of terms of the form

$$
1+p^{-2} f\left(\chi_{1}, \chi_{2}, \chi_{3}, p, z_{1}, z_{2}, z_{3}\right)
$$

with $f$ bounded, times some nonzero factors from primes dividing $P_{R}$ (namely, certain factors from the $\zeta$ and $L$-functions). The latter is a finite product of nonzero terms, each of which is between $1-p^{-1}$ and $1+p^{-1}$, and the former's logarithm's absolute value is dominated by $c_{R} \sum p^{-2}$ for some constant $c_{R}$, and thus it also cannot be zero. Thus $\eta(0,0,0)$ is bounded away from zero by a constant depending only on $R$, not on the characters or their conductors. In practice, it turns out to be enough to take $P_{R}=2 \times 3 \times 5 \times 7$, but for the sake of the proof it is enough to show that it exists.

If any of the characters are real, this isn't quite right, since then $L\left(\chi_{i}^{2}, 1\right)$ is infinite. Everything we have derived through (10.4) is still correct, so it is just a matter of evaluating the degree of the various poles (in particular, so long as the characters are distinct we get no poles from the shift of the $s$ contour, so $\log q$ never appears in the formula). As we have just seen, if any of the characters are complex then we only get contributions to the main term from those $P M$ 's in which they appear unconjugated. Thus in the remaining analysis, we can assume that $\chi_{A}=\chi_{1}$ and so forth.

Now suppose for a moment that $\chi_{1}$ is real but $\chi_{2}$ and $\chi_{3}$ are complex. Then (10.4) becomes

$$
\begin{aligned}
P M= & \frac{L\left(\chi_{1} \chi_{2}, 1\right) L\left(\chi_{1} \chi_{3}, 1\right) L\left(\chi_{2} \chi_{3}, 1\right)}{(2 \pi \mathrm{i})^{3}} \iiint \frac{L^{z_{1}+z_{2}+z_{3}} \eta\left(z_{1}, z_{2}, z_{3}\right)}{\zeta_{D_{1}}\left(1+z_{1}\right)} \times \\
& \times \frac{L\left(\chi_{1} \chi_{2}, 1+z_{1}+z_{2}\right) L\left(\chi_{1} \chi_{3}, 1+z_{1}+z_{3}\right) L\left(\chi_{2} \chi_{3}, 1+z_{2}+z_{3}\right)}{L\left(\chi_{1} \chi_{2}, 1+z_{1}\right) L\left(\chi_{1} \chi_{3}, 1+z_{1}\right) \prod_{i=2}^{3} L\left(\chi_{i} \chi_{1}, 1+z_{i}\right) L\left(\chi_{i} \chi_{2}, 1+z_{i}\right) L\left(\chi_{i} \chi_{3}, 1+z_{i}\right)} \times \\
& \times \frac{\mathrm{d} z_{1} \mathrm{~d} z_{2} \mathrm{~d} z_{3}}{z_{1}^{4} z_{2}^{4} z_{3}^{4}} .
\end{aligned}
$$

The $z_{2}$ and $z_{3}$ contours can be shifted as before, giving the same powers of $\log L$, and the $z_{1}$ contour can also be shifted, and also gives only a triple pole, since the $\zeta_{D_{1}}\left(1+z_{1}\right)^{-1}$ provides a zero. Thus we wind up with a main term of

$$
P M=\eta(0,0,0) \frac{\phi\left(D_{1}\right) / D_{1}}{L\left(\chi_{2}^{2}, 1\right) L\left(\chi_{3}^{2}, 1\right)}(\log L)^{8} .
$$

Similarly, we will always get a main term of the form

$$
\eta(0,0,0) \prod_{\chi_{i}^{2}=1} \frac{\phi\left(D_{i}\right)}{D_{i}}\left(\prod_{\chi_{i}^{2} \neq 1} L\left(\chi_{i}^{2}, 1\right)\right)^{-1}(\log L)^{3 c+2 r},
$$


where there are $r$ real characters and $c$ complex characters. Since we are only concerned with the existence of such a constant, not its precise value, this is enough.

\subsection{THE FOURTH MOMENT}

We now assume that $\chi$ is complex, of squarefree level $D>1$, such that $\chi^{2}$ is still primitive. We wish to compute (and bound)

$$
M(\chi)=\sum_{f \in S_{2}^{*}(q)}^{h}|L(f \cdot \chi, 1 / 2)|^{4}\left|M_{\chi}(f)\right|^{4},
$$

where the coefficients of $M_{\chi}(f)$ are defined by (10.1). Using (2.2) (and $(\ell, q D)=1$ ) we have

$$
\begin{aligned}
\left|M_{\chi}(f)\right|^{4}= & \frac{1}{(2 \pi \mathrm{i})^{4}} \int_{(2)^{4}} L\left(\chi^{2}, 1+z_{1}+z_{3}\right) L\left(\bar{\chi}^{2}, 1+z_{2}+z_{4}\right) \times \\
& \times \prod_{\substack{i j \\
(i, j) \neq(1,3),(2,4)}} \zeta^{(D)}\left(1+z_{i}+z_{j}\right) \eta(z) \times \sum_{(\ell, q D)=1} \frac{\lambda f(\ell)}{\ell^{1 / 2}} v(z, \ell) \times \\
& \times\left(\sum_{\substack{\ell=m_{1} m_{2} \times \\
m_{3} m_{4}}} \chi\left(m_{1} m_{3}\right) \bar{\chi}\left(m_{2} m_{4}\right) \frac{\mu\left(m_{1}\right) \mu\left(m_{2}\right) \mu\left(m_{3}\right) \mu\left(m_{4}\right)}{m_{1}^{z_{1}} m_{2}^{z_{2}} m_{3}^{z_{3}} m_{4}^{z_{4}}}\right) \frac{L^{z} \mathrm{~d} z}{\boldsymbol{z}^{n}} .
\end{aligned}
$$

Thus $\left|M_{\chi}(f)\right|^{4}=\sum \lambda_{f}(\ell) x(\ell) \ell^{-1 / 2} \quad$ with $\quad x(\ell) \quad$ only supported on $\ell<L^{4}$, $\left(\ell, q D P_{R}\right)=1$. Shifting the $z_{i}$ contours to $\Re e z_{i}=\varepsilon / 4$ shows that $x(\ell) \ll_{\varepsilon} \ell^{\varepsilon}$ for any $\varepsilon>0$. Proposition 7.1 implies that

$$
M(\chi)=\sum_{\substack{\ell \leqslant L^{4} \\(\ell, q D)=1}} \frac{1}{\ell^{1 / 2}} x(\ell)\left[M^{D}(\ell)+M^{O D}(\ell)+M^{O O D}(\ell)\right]+o_{D}(1)
$$

so long as $L<q^{1 / 60-\delta}$ for some fixed $\delta$.

\subsection{THE DIAGONAL AND OFF-DIAGONAL TERMS}

We recall from (7.9) that the diagonal and off-diagonal terms take the following form:

$$
\begin{aligned}
M^{D}(\ell)+M^{O D}(\ell)= & \operatorname{Res}_{s=0} \frac{\varphi(q)}{q \ell^{1 / 2}} \frac{4}{2 \pi \mathrm{i}} \int_{(2)} G(s) G(t) \Gamma^{2}(1+s) \Gamma^{2}(1+t) \zeta^{(D)}(1+2 s) \times \\
& \times \zeta^{(q D)}(1+2 t) \times \sum_{d e=\ell} \frac{1}{d^{s} e^{t}} \sum_{n \geqslant 1} \frac{\tau_{\chi}(d n) \tau_{\chi}(e n)}{n^{1+s+t}}\left(\frac{q D^{2}}{4 \pi^{2}}\right)^{s+t} \frac{\mathrm{dt}}{s t} .
\end{aligned}
$$


We have

$$
\sum_{d e=\ell} \frac{1}{d^{s} e^{t}} \sum_{n \geqslant 1} \frac{\tau_{\chi}(d n) \tau_{\chi}(e n)}{n^{1+s+t}}=\sum_{a b d=\ell} \frac{1}{d^{s}} \frac{\mu(a) \tau_{\chi}(b)}{a^{1+s+2 t} b^{t}} \sum_{n \geqslant 1} \frac{\tau_{\chi}(a d n) \tau_{\chi}(n)}{n^{1+s+t}}
$$

so that, using the identity $\tau_{\chi}\left(p^{k+\alpha}\right)=\chi\left(p^{\alpha}\right) \tau_{\chi}\left(p^{k}\right)+\bar{\chi}\left(p^{k}\right) \tau_{\chi}\left(p^{\alpha}\right)-\chi\left(p^{\alpha}\right) \bar{\chi}\left(p^{k}\right)$,

$$
\begin{aligned}
\sum_{n \geqslant 1} \frac{\tau_{\chi}(a d n) \tau_{\chi}(n)}{n^{s}}=\frac{\zeta^{(D)}(s)^{2} L\left(\chi^{2}, s\right) L\left(\bar{\chi}^{2}, s\right)}{\zeta^{(D)}(2 s)} \times \\
\quad \times \prod_{\substack{p^{\alpha}|| a d \\
\alpha>0}}\left(\tau_{\chi}\left(p^{\alpha}\right)+\chi\left(p^{\alpha}\right)\left(1-\frac{\zeta_{p}(s) L_{p}\left(\chi^{2}, s\right)}{\zeta_{p}(2 s)}\right)\right) .
\end{aligned}
$$

Hence

$$
\begin{aligned}
& M^{D}(\ell)+M^{O D}(\ell) \\
& =\operatorname{Res}_{s=0} \frac{\varphi(q)}{q \ell^{1 / 2}} \frac{4}{2 \pi \mathrm{i}} \int_{(2)} G(s) G(t) \Gamma^{2}(1+s) \Gamma^{2}(1+t) \zeta^{(D)}(1+2 s) \zeta^{(q D)}(1+2 t) \times \\
& \times \frac{\zeta^{(D)}(1+s+t)^{2} L\left(\chi^{2}, 1+s+t\right) L\left(\bar{\chi}^{2}, 1+s+t\right)}{\zeta^{(D)}(2+2 s+2 t)} \Psi^{\prime}(s, t ; \ell)\left(\frac{q D^{2}}{4 \pi^{2}}\right)^{s+t} \frac{\mathrm{d} t}{s t},
\end{aligned}
$$

with

$$
\begin{aligned}
\Psi^{\prime}(s, t ; \ell):= & \sum_{a b d=\ell} \frac{1}{d^{s}} \frac{\mu(a) \tau_{\chi}(b)}{a^{1+s+2 t} b^{t}} \times \\
& \times \prod_{\substack{p^{\alpha}|| a d \\
\alpha>>0}}\left(\tau_{\chi}\left(p^{\alpha}\right)+\chi\left(p^{\alpha}\right)\left(1-\frac{\zeta_{p}(1+s+t) L_{p}\left(\chi^{2}, 1+s+t\right)}{\zeta_{p}(2+2 s+2 t)}\right)\right) .
\end{aligned}
$$

One can show that $\Psi^{\prime}(0,0, \ell)=\Psi(0,0, \ell)$ where $\Psi(0,0, \ell)$ is the multiplicative function defined in Section 9.2 (see (9.8)), this is important for the problem of computing an explicit lower bound for the proportion of non-vanishing.

Inserting (10.10), we find that the contribution of the diagonal and off-diagonal terms to the fourth moment takes the form

$$
\begin{aligned}
M^{D O D}(\chi)= & \frac{4}{2 \pi \mathrm{i}} \int_{(2)} \operatorname{Res}_{s=0} \frac{1}{(2 \pi \mathrm{i})^{4}} \int_{(2)^{4}} \eta(s, t, \boldsymbol{z}) G(s) G(t) \Gamma^{2}(1+s) \Gamma^{2}(1+t) \times \\
& \times \frac{L\left(\chi^{2}, 1+z_{1}+z_{3}\right) L\left(\bar{\chi}^{2}, 1+z_{2}+z_{4}\right) L\left(\chi^{2}, 1+s+t\right) L\left(\bar{\chi}^{2}, 1+s+t\right)}{\prod_{i=1}^{4} L\left(\bar{\chi}^{2(-1)^{i}}, 1+s+z_{i}\right) L\left(\chi^{2(-1)^{i}}, 1+t+z_{i}\right)} \times \\
& \times \prod_{\substack{i<j \\
(i, j) \neq(1,3),(2,4)}} \zeta^{(D)}\left(1+z_{i}+z_{j}\right) \frac{\zeta^{(D)}(1+s+t)^{2} \zeta^{(D)}(1+2 s) \zeta^{(q D)}(1+2 t)}{\prod_{i=1}^{4} \zeta^{(D)}\left(1+s+z_{i}\right) \zeta^{(D)}\left(1+t+z_{i}\right)} \times \\
& \times\left(\frac{q D^{2}}{4 \pi^{2}}\right)^{s+t} \frac{L^{z} \mathrm{~d} z}{z^{n}} \frac{\mathrm{d} t}{s t} .
\end{aligned}
$$


We shift the $z$ contours to $\Re e z_{i}=1 / 4$ and the $t$-contour to $\Re e t=-1 / 4$, passing a pole at $t=0$. The resulting integral is bounded by $\ll L q^{-1 / 4}$ which is admissible. Thus we have

$$
\begin{aligned}
M^{D O D}(\chi)= & \frac{4}{(2 \pi \mathrm{i})^{4}} \int_{(2)^{4}} \operatorname{Res}_{s=t=0} \frac{\left(q D^{2} / 4 \pi^{2}\right)^{s+t}}{s t} \eta(s, t, z) G(s) G(t) \Gamma^{2}(1+s) \Gamma^{2}(1+t) \times \\
& \times \frac{L\left(\chi^{2}, 1+z_{1}+z_{3}\right) L\left(\bar{\chi}^{2}, 1+z_{2}+z_{4}\right) L\left(\chi^{2}, 1+s+t\right) L\left(\bar{\chi}^{2}, 1+s+t\right)}{\prod_{i=1}^{4} t L\left(\bar{\chi}^{2(-1)^{i}}, 1+s+z_{i}\right) L\left(\chi^{2(-1)^{i}}, 1+t+z_{i}\right)} \times \\
& \times \prod_{\substack{i<j \\
(i, j) \neq(1,3),(2,4)}} \zeta^{(D)}\left(1+z_{i}+z_{j}\right) \times \\
& \times \frac{\zeta^{(D)}(1+s+t)^{2} \zeta^{(D)}(1+2 s) \zeta^{(q D)}(1+2 t)}{\prod_{i=1}^{4} \zeta^{(D)}\left(1+s+z_{i}\right) \zeta^{(D)}\left(1+t+z_{i}\right)} \frac{L^{z} \mathrm{~d} z}{z^{n}}
\end{aligned}
$$

As in [KMV2] 5.3, everything can be evaluated by shifting the $z_{i}$ contours just past the line $\Re z_{i}=0$, the only important contribution coming from the following 5 sequences of poles

$$
\begin{aligned}
& \left(z_{1}=0, z_{2}=0, z_{3}=0, z_{4}=0\right), \\
& \left(z_{1}=0, z_{2}=-z_{3}, z_{4}=z_{3}, z_{3}=0\right), \\
& \left(z_{1}=-z_{2}, z_{3}=z_{2}, z_{2}=0, z_{4}=0\right), \\
& \left(z_{1}=-z_{2}, z_{3}=0, z_{4}=z_{2}, z_{2}=0\right), \\
& \left(z_{1}=-z_{3}, z_{2}=0, z_{4}=z_{3}, z_{3}=0\right) .
\end{aligned}
$$

All other sequences contribute lower powers of $\log q$, so long as $n \geqslant 4$. The important question is what power of $\log L \sim \log q$ comes out of this calculation. This is simply a matter of counting poles: the $\zeta$ functions in the numerator contribute eight poles, those in the denominator remove eight, and the combined powers of $s, t$, and $z_{i}$ contribute $4 n+2$. Thus the leading power of $\log q$ or $\log L$ will be $(\log q)^{4 n-4}$. Thus, taking $n=4$, the fourth moment is dominated by $(\log q)^{12}$ times a constant of the form

$$
c \frac{L\left(\chi^{2}, 1\right) L\left(\bar{\chi}^{2}, 1\right) L\left(\chi^{2}, 1\right) L\left(\bar{\chi}^{2}, 1\right)}{\prod_{i=1}^{4} L\left(\bar{\chi}^{2(-1)^{i}}, 1\right) L\left(\chi^{2(-1)^{i}}, 1\right)} \zeta_{D}(1)^{4} \frac{\left.\zeta_{D}(1)^{2} \zeta_{D}(1) \zeta_{D}(1)\right)}{\prod_{i=1}^{4} \zeta_{D}(1) \zeta_{D}(1)}=\frac{c}{\left|L\left(\chi^{2}, 1\right)\right|^{4}}
$$

where $c$ is bounded from above independent of $\chi$. Recall, (10.5), that the main term of the third moment was proportional to $(\log q)^{9}$ times a constant of the form $c^{\prime} / L\left(\chi_{1}^{2}, 1\right) L\left(\chi_{2}^{2}, 1\right) L\left(\chi_{3}^{2}, 1\right)$ when all the characters are complex. 


\subsection{THE OFF-OFF-DIAGONAL TERMS}

Using (9.5) and (9.6), we have

$$
\begin{aligned}
M^{O O D,++}(\chi)= & \frac{\bar{\chi}^{2}(q) \mu(D) G\left(\chi^{-2}\right) G(\bar{\chi})^{2} L\left(\chi^{2}, 1\right)^{6}}{L\left(\chi^{4}, 2\right) D^{2}} \frac{1}{(2 \pi \mathrm{i})^{4}} \int_{(2)^{4}} \eta(z) \times \\
& \times \frac{L\left(\chi^{2}, 1+z_{1}+z_{3}\right) L\left(\bar{\chi}^{2}, 1+z_{2}+z_{4}\right)}{\left[L\left(\chi^{2}, 1+z_{1}\right) L\left(\bar{\chi}^{2}, 1+z_{3}\right) \zeta^{(D)}\left(1+z_{2}\right) \zeta^{(D)}\left(1+z_{3}\right)\right]^{4}} \times \\
& \times \prod_{\substack{i<j \\
(i, j) \neq(1,3),(2,4)}} \zeta^{(D)}\left(1+z_{i}+z_{j}\right) \frac{L^{z} \mathrm{~d} z}{z^{n}} .
\end{aligned}
$$

Again, all we need is bounds in terms of powers of $\log L$ arising from poles in the $z$ variables, since there are no powers of $\log q$ coming from the $s$ or $t$ residues. The numerator gives four $\zeta$ functions, the denominator eight, so when combined with the powers of $z_{i}$ there are a total of $4 n-4$ poles in four variables, giving a leading term of $(\log L)^{4 n-8}=(\log L)^{8}$ for $n=4$. Note that this is a lower power than we got from the diagonal and off-diagonal terms.

Next we consider the +- term, which from (9.10) is

$$
\begin{aligned}
& M^{O O D,+-}(\chi)=\frac{\chi(-1)\left|L\left(\chi^{2}, 1\right)\right|^{2}}{\zeta^{(D)}(2)} \frac{1}{(2 \pi \mathrm{i})^{4}} \iint_{(2)^{4}} \times \\
& \times \operatorname{Res}_{s, t=0} \eta(s, t, \boldsymbol{z}) \frac{G(s) G(t)}{s t} \prod_{ \pm} \Gamma(1 \pm s) \Gamma(1 \pm t) \prod_{ \pm, \pm} \zeta^{(D)}(1 \pm s \pm t) \times \\
& \times \frac{L\left(\chi^{2}, 1+z_{1}+z_{3}\right) L\left(\bar{\chi}^{2}, 1+z_{2}+z_{4}\right)}{\prod_{ \pm} L\left(\chi^{2}, 1+z_{1} \pm t\right) L\left(\chi^{2}, 1+z_{3} \pm t\right) L\left(\bar{\chi}^{2}, 1+z_{2} \pm s\right) L\left(\bar{\chi}^{2}, 1+z_{4} \pm s\right)} \times \\
& \times \frac{\prod_{i<j \neq(1,3),(2,4)} \zeta^{(D)}\left(1+z_{i}+z_{j}\right)}{\prod_{ \pm} \zeta\left(1+z_{1} \pm s\right) \zeta\left(1+z_{3} \pm s\right) \zeta\left(1+z_{2} \pm t\right) \zeta\left(1+z_{4} \pm t\right)} \frac{L^{z} \mathrm{~d} z}{z^{n} .}
\end{aligned}
$$

Using the same arguments, it is enough to count the factors of $\log L$ arising from taking the various $s, t, z$ poles, which is $4 n-4$. Thus for $n=4$ the off-off diagonal terms produce a main term proportional to $(\log L)^{12}$ times a constant of the form $c^{\prime \prime} /\left(\left|L\left(\chi^{2}, 1\right)\right|^{4}\right)$.

Remark. It is instructive to note that althought the off-off-diagonal terms are the most complicated of the main terms, they contribute less: for the untwisted fourth moment of Proposition 1.2, the contribution of these terms is by 4 powers of $\log q$ smaller than that of the diagonal terms. For the mollified fourth moment, setting $L=q^{\Delta}$ we see that the diagonal and off-diagonal terms contribute by (essentially) $\simeq \log ^{12} q$, while the $M^{O O D++}$ terms contribute by $\ll \Delta^{8} \log ^{8} q$ which is negligeable, and $M^{O O D-+}$ by $\simeq \Delta^{12} \log ^{12} q$ which althought contributing, becomes smaller for smaller $\Delta$ (note that for Theorem 1.1 we cannot take $\Delta$ arbitrarly small in view of the third moment). Note also that, the discrepancy in the contributions of the $M^{O O D-+}$ 
and the $M^{O O D++}$ terms can be guessed already by looking at (8.9) and (8.10): in (8.9) there is an oscillating factor $G\left(\chi^{2}, h ; D\right)$ which is not present in (8.10).

\subsection{CONCLUSION OF THE PROOF OF THEOREM 1.1}

Thus, under the assumption that all the characters are complex, we have shown that that there exist mollifiers $M_{i}(f)$ such that

$$
\begin{aligned}
& \left|\sum_{f}^{h} L\left(f \cdot \chi_{1}, \frac{1}{2}\right) M_{1}(f) L\left(f \cdot \chi_{2}, \frac{1}{2}\right) M_{2}(f) L\left(f \cdot \chi_{3}, \frac{1}{2}\right) M_{3}(f)\right| \\
& \quad \geqslant\left(c_{0}+\mathrm{o}(1)\right) \frac{(\log L)^{9}}{\left|\prod_{i=1}^{3} L\left(\chi_{i}^{2}, 1\right)\right|}
\end{aligned}
$$

and

$$
\sum_{f}^{h}\left|L\left(f \cdot \chi_{i}, \frac{1}{2}\right) M_{i}(f)\right|^{4} \leqslant\left(c_{i}+\mathrm{o}(1)\right) \frac{(\log q)^{12}}{\left|L\left(\chi_{i}^{2}, 1\right)\right|^{4}},
$$

where $c_{0}, c_{i}$ are absolute constants, $c_{0}>0$, and $L=q^{\Delta}$ for some positive fixed $\Delta$. This proves that

$$
\sum_{\substack{f, L\left(f, \mathcal{X}_{i}, 1 / 2\right) \neq \neq \\ i=1,2,3}}^{h} 1 \geqslant \frac{c_{0}^{4} \Delta^{4}}{c_{1} c_{2} c_{3}}+o(1),
$$

and we conclude the proof as explained in Section 4. Suppose now that $\chi_{1}$ is the trivial character but $\chi_{2}$ and $\chi_{3}$ are complex: from (10.7), we have

$$
\left|\sum_{f}^{h} L\left(f, \frac{1}{2}\right) M(f) L\left(f \cdot \chi_{2}, \frac{1}{2}\right) M_{2}(f) L\left(f \cdot \chi_{3}, \frac{1}{2}\right) M_{3}(f)\right| \geqslant\left(c_{0}+\mathrm{o}(1)\right) \frac{(\log L)^{8}}{\left|L\left(\chi_{2}^{2}, 1\right) L\left(\chi_{3}^{2}, 1\right)\right|} .
$$

The necessary upper bound on the fourth moment for the trivial character is provided by [KMV2], Theorem 1.4, which gives (with our present choice of mollifier)

$$
\sum_{f}^{h}\left|L\left(f, \frac{1}{2}\right) M(f)\right|^{4} \leqslant\left(c_{1}+o(1)\right)(\log q)^{8} .
$$

Since $4 \times 8=8+12+12$, we still have (10.15).

\section{Variant for Real Characters}

Finally, we discuss the variant, Theorem 1.4. Recall that this involves the case in which $\chi_{1}$ is trivial, $\chi_{2}$ is real, and $\chi_{3}$ is complex. We assume also that $D_{2}, D_{3}$ are squarefree and that $\chi_{3}^{2}$ is primitive. For $q$ prime such that $\chi_{2}(-q)=1$, the first case of Theorem 1.4 follows from the inequality 


$$
\begin{aligned}
& \left(\sum_{f \in S_{2}^{*}(q)}^{h} L_{1,2}(f) L_{3}(f)\right)^{4} \\
& \quad \leqslant\left(\sum_{\substack{f \in S_{2}^{*}(q) \\
L_{1,2}(f) L_{3}(f) \neq 0}}^{h} 1\right)\left(\sum_{f \in S_{2}^{*}(q)}^{h}\left|L_{1,2}(f)\right|^{2}\right)^{2}\left(\sum_{f \in S_{2}^{*}(q)}^{h}\left|L_{3}(f)\right|^{4}\right),
\end{aligned}
$$

where

$$
L_{1,2}(f)=M_{1}(f) M_{2}(f) L\left(f, \frac{1}{2}\right) L\left(f \cdot \chi_{2}, \frac{1}{2}\right)
$$

and

$$
L_{3}(f)=M_{3}(f) L\left(f \cdot \chi_{3}, \frac{1}{2}\right) .
$$

The only remaining question is to evaluate the second moment

$$
\sum_{f}^{h}\left(L\left(f, \frac{1}{2}\right) L\left(f \cdot \chi_{2}, \frac{1}{2}\right)\right)^{2} \lambda_{f}(\ell) .
$$

This calculation winds up being quite similar to the one we have already done, but avoids many of the pitfalls that we have encountered above because now $1 * \chi_{2}(n)$ represents the coefficients of an Eisenstein series of square-free level (for example, it is instructive to compare the calculations of Appendix B.2 which is used here with those of Appendix B.1). The remainder terms have exactly the same size as before, and the main term, gets contributions from the diagonal, off-diagonal, and offoff-diagonal which gives that the averages of $\left|L_{1,2}(f)\right|^{2}$ goes as $(\log q)^{8}$, which is precisely the correct power to make the proportion of nonvanishing triple products go as a constant: recall that the lefthand side of $(11.1)$ goes as $(\log L)^{7 \times 4}$, and the last factor of the right-hand side goes as $(\log q)^{12}$. Rather than reproduce all the calculations here and lengthen this paper even further, we merely present the asymptotics for the case $\ell=1$, which has independent interest. The average (using the harmonic weights) of the square of the $k$ th derivative of $\Lambda\left(f, \frac{1}{2}\right) \Lambda\left(f \cdot \chi_{2}, \frac{1}{2}\right)$ is the sum of an error term of the usual size, plus two main terms $M^{D O D}+M^{O O D}$, where

$$
\begin{aligned}
M^{D O D}= & \frac{4(k !)^{2}}{(2 \pi \mathrm{i})^{2}} \int_{(3)} \int_{(2)} \frac{G(t) \Gamma(1+t)^{2} L\left(\chi_{2}, 1+2 t\right)}{s^{k+1} t^{k+1}}\left(\frac{q D}{4 \pi^{2}}\right)^{t}(F(s, t)+ \\
& +\chi(-q) F(-s, t)) \mathrm{d} s \mathrm{~d} t,
\end{aligned}
$$

where

$$
\begin{aligned}
F(s, t)= & G(s) \Gamma(1+s)^{2} L\left(\chi_{2}, 1+2 s\right)\left(\frac{q D}{4 \pi^{2}}\right)^{s} \times \\
& \times \frac{\zeta(1+s+t) \zeta^{(D)}(1+s+t) L\left(\chi_{2}, 1+s+t\right)^{2}}{L\left(\chi_{2}, 2+2 s+2 t\right)},
\end{aligned}
$$


and

$$
\begin{aligned}
M^{O O D}= & \frac{4(k !)^{2} L\left(\chi_{2}, 1\right)^{2}}{\zeta(2)(2 \pi \mathrm{i})^{2}} \int_{(3)} \int_{(2)} \frac{G(s) G(t) \prod_{ \pm} \Gamma(1 \pm s) \Gamma(1 \pm t)}{t^{k+1} s^{k+1}} \times \\
& \times\left[\left(\frac{D^{s+t}}{\zeta_{D}(1+s+t)}+\frac{D^{-s-t}}{\zeta_{D}(1-s-t)}\right) \prod_{ \pm} \zeta(1 \pm(s+t)) L\left(\chi_{2}, 1 \pm(s-t)\right)+\right. \\
& +\chi(-q)\left(\frac{D^{-s+t}}{\zeta_{D}(1-s+t)}+\frac{D^{s-t}}{\zeta_{D}(1+s-t)}\right) \times \\
& \left.\times \prod_{ \pm} L\left(\chi_{2}, 1 \pm(s+t)\right) \zeta(1 \pm(s-t))\right] \mathrm{d} s \mathrm{~d} t .
\end{aligned}
$$

Notice that in both cases, the parity of the integrands is odd precisely when the relevant derivative can be nonzero, that is, when $\chi(-q)=1$ for even $k$ and when $\chi(-q)=-1$ for odd $k$. Thus these expressions can be evaluated by taking residues at $s=0$ and then $t=0$ in the usual fashion. In particular, for $k=0$ and $\chi(-q)=1$, the asymptotics go as $P_{\chi_{2}}(\log q)$, where $P_{\chi_{2}}$ is a quadratic polynomial with coefficients depending on $\chi_{2}$.

\section{Appendix A. Summation Formulae}

In this section we derive a Poisson-like summation formula for the convolution of two distinct primitive Dirichlet characters. These results are standard but we couldn't find them in the existing literature with the required degree of generality. Our methods essentially follow those of [J] but there are other ways to obtain this formula. We first start with a very general proposition obtained by double application of Poisson summation.

PROPOSITION A.1. Given $c_{1}, c_{2} \geqslant 1$ two integers and $G$ a complex valued function on $\left(\mathbf{Z} / c_{1} \mathbf{Z}\right) \times\left(\mathbf{Z} / c_{2} \mathbf{Z}\right)$. Let

$$
H\left(m_{1}, m_{2}\right)=\sum_{r_{1}\left(c_{1}\right)} \sum_{r_{2}\left(c_{2}\right)} G\left(r_{1}, r_{2}\right) e\left(\frac{m_{1} r_{1}}{c_{1}}+\frac{m_{2} r_{2}}{c_{2}}\right)
$$

denote its discrete Fourier transform, and assume that $H\left(-m_{1},-m_{2}\right)=v H\left(m_{1}, m_{2}\right)$ for some $v \in\{ \pm 1\}$. Let $F(x)$ be a smooth function on $(0, \infty)$, vanishing in a neighborhood of 0 , such that $F$ and all its derivatives have rapid decacy at $\infty$. Then

$$
\begin{aligned}
& \sum_{m_{1}, m_{2} \geqslant 1} G\left(m_{1}, m_{2}\right) F\left(m_{1} m_{2}\right) \\
& \quad=\operatorname{Res}_{s=1} \frac{\hat{F}(1+s)}{\left(c_{1} c_{2}\right)^{1+s}}\left(\frac{H(0,0)}{s^{2}}+\frac{1}{s}\left(H^{-}+H^{+}\right)\right)+
\end{aligned}
$$




$$
\begin{aligned}
& +\frac{v}{c_{1} c_{2}} \sum_{m_{1}, m_{2} \geqslant 1} H\left(m_{1}, m_{2}\right) \int_{0}^{\infty} F(x) J^{v}\left(4 \pi \sqrt{\frac{m_{1} m_{2} x}{c_{1} c_{2}}}\right) \mathrm{d} x+ \\
& +\frac{1}{c_{1} c_{2}} \sum_{m_{1}, m_{2} \geqslant 1} H\left(m_{1},-m_{2}\right) \int_{0}^{\infty} F(x) K^{v}\left(4 \pi \sqrt{\frac{m_{1} m_{2} x}{c_{1} c_{2}}}\right) \mathrm{d} x,
\end{aligned}
$$

where $\hat{F}(s)$ is the Mellin transform of $F, c_{1}=\left[c, D_{1}\right], c_{2}=\left[c, D_{2}\right]$,

$$
\begin{aligned}
& H^{-}=\sum_{r_{1}\left(c_{1}\right)} \sum_{r_{2}\left(c_{2}\right)} G\left(r_{1}, r_{2}\right)\left(-\frac{\Gamma^{\prime}}{\Gamma}\left(\frac{r_{1}}{c_{1}}\right)\right), \\
& H^{+}=\sum_{r_{1}\left(c_{1}\right)} \sum_{r_{2}\left(c_{2}\right)} G\left(r_{1}, r_{2}\right)\left(-\frac{\Gamma^{\prime}}{\Gamma}\left(\frac{r_{2}}{c_{2}}\right)\right), \\
& J^{+}(x)=-2 \pi Y_{0}(x) ; \quad J^{-}(x)=2 \pi \mathrm{i} J_{0}(x) ; \quad K^{+}(x)=4 K_{0}(x) ; \quad K^{-}(x)=0 .
\end{aligned}
$$

Proof. We rewrite the sum as

$$
\sum_{m_{1}, m_{2}} G\left(m_{1}, m_{2}\right) F\left(m_{1} m_{2}\right)=\sum_{r_{1}\left(c_{1}\right)} \sum_{r_{2}\left(c_{2}\right)} G\left(r_{1}, r_{2}\right) \sum_{m_{1} \equiv r_{1}} \sum_{m_{2} \equiv r_{2}} F\left(m_{1} m_{2}\right) .
$$

Taking the Mellin transform of $F$ and using the Hurwitz zeta function

$$
\zeta(s, \alpha)=\sum_{m \geqslant 1}(m+\alpha)^{-s},
$$

this becomes

$$
\sum_{r_{1}=1}^{c_{1}} \sum_{r_{2}=1}^{c_{2}} G\left(r_{1}, r_{2}\right) \frac{1}{2 \pi \mathrm{i}} \int_{(3)} \hat{F}(s) \frac{1}{\left(c_{1} c_{2}\right)^{s}} \zeta\left(s, \frac{r_{1}}{c_{1}}\right) \zeta\left(s, \frac{r_{2}}{c_{2}}\right) \mathrm{d} s .
$$

We shift the contour to the left, crossing over poles at $s=1$, then use the functional equation of $\zeta(s, \alpha)$ to convert to convergent sums.

A.1. THE POLES AT $s=1$

If $s$ is near 1 ,

$$
\zeta(s, \alpha)=\frac{1}{s-1}-\frac{\Gamma^{\prime}}{\Gamma}(\alpha)+\mathrm{O}(s-1)
$$

The portion of (A.3) coming from the pole is thus

$$
\sum_{r_{1}=1}^{c_{1}} \sum_{r_{2}=1}^{c_{2}} G\left(r_{1}, r_{2}\right) \operatorname{Res}_{s=1} \hat{F}(s) \frac{1}{\left(c_{1} c_{2}\right)^{s}}\left(\frac{1}{(s-1)^{2}}-\frac{1}{s-1}\left(\frac{\Gamma^{\prime}}{\Gamma}\left(\frac{r_{1}}{c_{1}}\right)+\frac{\Gamma^{\prime}}{\Gamma}\left(\frac{r_{2}}{c_{2}}\right)\right)\right) .
$$

giving the first term in (A.2). 


\section{A.2. THE SHIFTED INTEGRAL}

We recall the functional equation (see [Da])

$$
\zeta(s, \alpha)=\frac{2 \Gamma(1-s)}{(2 \pi)^{1-s}} \sum_{m=1}^{\infty} \frac{\sin 2 \pi(m \alpha+s / 4)}{m^{1-s}} .
$$

Thus upon shifting the contour in (A.3) to Res $=-2$ and replacing $s$ with $1-s$, we have the following contour integral:

$$
\begin{aligned}
& \frac{4}{c_{1} c_{2}} \sum_{r_{1}=1}^{c_{1}} \sum_{r_{2}=1}^{c_{2}} G\left(r_{1}, r_{2}\right) \frac{1}{2 \pi \mathrm{i}} \int_{(3)} \hat{F}(1-s) \Gamma(s)^{2}\left(\frac{c_{1} c_{2}}{4 \pi^{2}}\right)^{s} \sum_{m_{1}, m_{2}} \frac{1}{m_{1}^{s} m_{2}^{s}} \times \\
& \quad \times \frac{1}{4}\left(e\left(-\frac{s}{2}+\frac{m_{1} r_{1}}{c_{1}}+\frac{m_{2} r_{2}}{c_{2}}\right)+e\left(\frac{s}{2}-\frac{m_{1} r_{1}}{c_{1}}-\frac{m_{2} r_{2}}{c_{2}}\right)+e\left(\frac{m_{1} r_{1}}{c_{1}}-\frac{m_{2} r_{2}}{c_{2}}\right)+\right. \\
& \left.\quad+e\left(-\frac{m_{1} r_{1}}{c_{1}}+\frac{m_{2} r_{2}}{c_{2}}\right)\right) \mathrm{d} s .
\end{aligned}
$$

Note that $H\left(-m_{1},-m_{2}\right)=v H\left(m_{1}, m_{2}\right)$, so the contour integral's contribution to (A.3) is

$$
\begin{aligned}
& \frac{1}{c_{1} c_{2}} \sum_{m_{1}, m_{2}} \frac{1}{2 \pi \mathrm{i}} \int_{(3)} \hat{F}(1-s) \Gamma(s)^{2}\left(\frac{c_{1} c_{2}}{4 \pi^{2} m_{1} m_{2}}\right)^{s}\left(c^{v}(s) H\left(m_{1}, m_{2}\right)+\right. \\
& \left.\quad+(1+v) H\left(m_{1},-m_{2}\right)\right) \mathrm{d} s,
\end{aligned}
$$

with, $c^{+}(s)=2 \cos (\pi s)$, and $c^{-}(s)=-2 \mathrm{i} \sin (\pi s)$. We then shift the integration contour to $\Re e s=1 / 8$, open the Mellin transform $\hat{F}(1-s)$ and use the formulas (see [EMOT] Vol 1. 7.3 (17), (19), (23)), valid for $0<\sigma<1 / 4$,

$$
\begin{aligned}
& \frac{1}{2 \pi \mathrm{i}} \int_{(\sigma)} \Gamma^{2}(s) x^{-s} \mathrm{~d} s=2 K_{0}(2 \sqrt{x}), \frac{1}{2 \pi \mathrm{i}} \int_{(\sigma)} \Gamma^{2}(s) \cos (\pi s) x^{-s} \mathrm{~d} s=-\pi Y_{0}(2 \sqrt{x}), \\
& \frac{1}{2 \pi \mathrm{i}} \int_{(\sigma)} \Gamma^{2}(s) \sin (\pi s) x^{-s} \mathrm{~d} s=\pi J_{0}(2 \sqrt{x}) .
\end{aligned}
$$

This completes the proof of Proposition A.1.

\section{A.3. CONVOLUTION OF DIRICHLET CHARACTERS}

We apply Proposition A.1 to the case

$$
G\left(m_{1}, m_{2}\right)=\chi_{1}\left(m_{1}\right) \chi_{2}\left(m_{2}\right)
$$

for $\chi_{1}$ and $\chi_{2}$ two primitive Dirichlet characters.

PROPOSITION A.2. Given $\chi_{1}, \chi_{2}$ distinct primitive Dirichlet characters of conductors $D_{1}$ and $D_{2}$, respectively. Let $F(x)$ be a smooth function on $(0, \infty)$, vanishing in a neighborhood of 0 , such that $F$ and all its derivatives have rapid decay at $\infty$. Given $c$ a positive integer, and a coprime with $c$, 


$$
\begin{aligned}
\sum_{m \geqslant 1} \chi_{1} * \chi_{2}(m) e\left(\frac{a m}{c}\right) F(m) & \\
= & \frac{\delta_{D_{2} \mid c}}{c} \chi_{1}\left(\frac{c}{D_{2}}\right) \bar{\chi}_{2}(a) G\left(\chi_{2}\right) L\left(1, \chi_{1} \bar{\chi}_{2}\right) \int_{0}^{\infty} F(x) \mathrm{d} x+ \\
& +\frac{\delta_{D_{1} \mid c}}{c} \chi_{2}\left(\frac{c}{D_{1}}\right) \bar{\chi}_{1}(a) G\left(\chi_{1}\right) L\left(1, \bar{\chi}_{1} \chi_{2}\right) \int_{0}^{\infty} F(x) \mathrm{d} x+ \\
& +\frac{\chi_{1} \chi_{2}(-1)}{c_{1} c_{2}} \sum_{m_{1}, m_{2}} H\left(a, m_{1}, m_{2} ; c\right) \times \\
& \times \int_{0}^{\infty} F(x) J^{ \pm}\left(4 \pi \sqrt{\frac{m_{1} m_{2} x}{c_{1} c_{2}}}\right) \mathrm{d} x+\frac{\chi_{2}(-1)}{c_{1} c_{2}} \times \\
& \times \sum_{m_{1}, m_{2}} H\left(-a, m_{1}, m_{2} ; c\right) \times \\
& \times \int_{0}^{\infty} F(x) K^{ \pm}\left(4 \pi \sqrt{\frac{m_{1} m_{2} x}{c_{1} c_{2}}}\right) \mathrm{d} x,
\end{aligned}
$$

where

$$
\begin{aligned}
& \pm=\chi_{1} \chi_{2}(-1), \quad c_{1}=\left[c, D_{1}\right], \quad c_{2}=\left[c, D_{2}\right], \\
& H\left(a, m_{1}, m_{2} ; c\right)=\sum_{r_{1}\left(c_{1}\right)} \sum_{r_{2}\left(c_{2}\right)} \chi_{1}\left(r_{1}\right) \chi_{2}\left(r_{2}\right) e\left(\frac{m_{1} r_{1}}{c_{1}}+\frac{m_{2} r_{2}}{c_{2}}\right) e\left(\frac{a r_{1} r_{2}}{c}\right), \\
& J^{+}(x)=-2 \pi Y_{0}(x), \quad J^{-}(x)=2 \pi \mathrm{i} J_{0}(x), \quad K^{+}(x)=4 K_{0}(x), \quad K^{-}(x)=0 .
\end{aligned}
$$

Proof. This is immediate from Proposition A.1 and the following two lemmas.

LEMMA A.3. If $\chi_{1}$ and $\chi_{2}$ are primitive characters modulo $D_{1}$ and $D_{2}$, respectively, and $(a, c)=1$, then if $\chi_{1}=\chi_{2}$ and $D_{1} \mid c$,

$$
\sum_{r_{1}=1}^{\left[c, D_{1}\right]} \sum_{r_{2}=1}^{\left.c, D_{2}\right]} \chi_{1}\left(r_{1}\right) \chi_{2}\left(r_{2}\right) e\left(\frac{a r_{1} r_{2}}{c}\right)=c \chi_{1}\left(\bar{a} \frac{c}{D_{1}}\right) G\left(\chi_{1}\right) \frac{\phi\left(D_{1}\right)}{D_{1}}
$$

but otherwise the sum is zero.

Proof. If $c_{1}:=\left[D_{1}, c\right]>c$, we may write $r_{1}=c s_{1}+t_{1}$, with $1 \leqslant t_{1} \leqslant c$ and $0 \leqslant s_{1}<c_{1} / c$, and the $s_{1}$ sum takes the form $\sum \chi_{1}\left(c s_{1}+r_{1}\right)=0$ since $\chi_{1}$ is primitive modulo $D_{1}$. Thus we have $c_{1}=c$, and by symmetry we also have $c_{2}=c$, so $D_{1}$ and $D_{2}$ must both divide $c$. We now write $r_{1}=D_{1} s_{1}+t_{1}$, with $1 \leqslant t_{1} \leqslant D_{1}$ and $0 \leqslant s_{1}<c / D_{1}$, so that the $s_{1}$ sum is

$$
\sum_{s_{1}<c / D_{1}} e\left(\frac{a r_{2} s_{1} D_{1}}{c}\right)=\frac{c}{D_{1}} \delta_{c / D_{1} \mid r_{2}}
$$


Thus the complete sum is

$$
\frac{c}{D_{1}} \sum_{t_{1}=1}^{D_{1}} \sum_{s_{2}=1}^{D_{1}} \chi_{1}\left(t_{1}\right) \chi_{2}\left(s_{2} \frac{c}{D_{1}}\right) e\left(\frac{a s_{2} t_{1}}{D_{1}}\right)
$$

This vanishes unless $\left(D_{2}, c / D_{1}\right)=1$, which is to say $D_{2} \mid D_{1}$. Since the original expression was symmetric in $D_{1}$ and $D_{2}$, we must also have $D_{1} \mid D_{2}$, so $D_{1}=D_{2}$. The $t_{1}$ sum is then

$$
\sum_{t_{1}=1}^{D_{1}} \chi_{1}\left(t_{1}\right) e\left(\frac{a s_{2} t_{1}}{D_{1}}\right)=\overline{\chi_{1}}\left(a s_{2}\right) G\left(\chi_{1}\right)
$$

leaving us with the expression

$$
\frac{c}{D_{1}} \chi_{2}\left(\frac{c}{D_{1}}\right) \overline{\chi_{1}}(a) G\left(\chi_{1}\right) \sum_{s_{2}=1}^{D_{1}} \chi_{2}\left(s_{2}\right) \overline{\chi_{1}}\left(s_{2}\right)
$$

which is zero unless $\chi_{1}=\chi_{2}$.

LEMMA A.4. With notation as above,

$$
-\sum_{r_{1}\left(c_{1}\right), r_{2}\left(c_{2}\right)} \chi_{1}\left(r_{1}\right) \chi_{2}\left(r_{2}\right) e\left(\frac{a r_{1} r_{2}}{c}\right) \frac{\Gamma^{\prime}}{\Gamma}\left(\frac{r_{1}}{c_{1}}\right)=\delta_{D_{2} \mid c} c_{1} \chi_{1}\left(\frac{c}{D_{2}}\right) \bar{\chi}_{2}(a) G\left(\chi_{2}\right) L\left(1, \chi_{1} \overline{\chi_{2}}\right)
$$

if $\chi_{1} \neq \chi_{2}$, and

$$
\begin{aligned}
& -\sum_{r_{1}, r_{2}} \chi_{1}\left(r_{1}\right) \chi_{2}\left(r_{2}\right) e\left(\frac{a r_{1} r_{2}}{c}\right) \frac{\Gamma^{\prime}}{\Gamma}\left(\frac{r_{1}}{c_{1}}\right) \\
& =\delta_{D_{1} \mid c} c \frac{\varphi\left(D_{1}\right)}{D_{1}} \chi_{1}\left(\frac{c}{D_{1}}\right) \bar{\chi}_{1}(a) G\left(\chi_{1}\right)\left(\log \left(D_{1}\right)+\gamma+\Psi\left(D_{1}\right)\right)
\end{aligned}
$$

if $\chi_{1}=\chi_{2}$, where $\Psi\left(D_{1}\right)=\sum_{p \mid D_{1}} \frac{\log p}{p-1}$.

Proof. We perform the $r_{2}$ sum first, and in a manner similar to the previous proof we obtain that the sum is zero unless $D_{2} \mid c$ in which case we are left with

$$
\frac{c}{D_{2}} G\left(\chi_{2}\right) \chi_{1}\left(\frac{c}{D_{2}}\right) \bar{\chi}_{2}(a) \sum_{s=1}^{D_{1} D_{2} /\left(c, D_{1}\right)} \chi_{1} \overline{\chi_{2}}(s) \frac{\Gamma^{\prime}}{\Gamma}\left(\frac{s}{D_{1} D_{2} /\left(c, D_{1}\right)}\right) .
$$

We now use the identity, obtained using

$$
\begin{aligned}
& -\frac{\Gamma^{\prime}(\alpha)}{\Gamma(\alpha)}=\frac{1}{\alpha}+\gamma+\sum_{k \geqslant 1} \frac{1}{k+\alpha}-\frac{1}{k}, \\
& \sum_{1 \leqslant r \leqslant c D} \chi(r)\left(-\frac{\Gamma^{\prime}}{\Gamma}\left(\frac{r}{c D}\right)\right)=\operatorname{res}_{s=0} \frac{(c D)^{1+s}}{s} L(\chi, 1+s) .
\end{aligned}
$$


Thus if $\chi_{1} \neq \chi_{2}$ the sum is

$$
\frac{c}{D_{2}} \frac{D_{1} D_{2}}{\left(D_{1}, c\right)} G\left(\chi_{2}\right) \chi_{1}\left(\frac{c}{D_{2}}\right) \bar{\chi}_{2}(a) L\left(1, \chi_{1} \overline{\chi_{2}}\right)
$$

the desired result, and similar if $\chi_{1}=\chi_{2}$.

\section{Appendix B. An Exponential Sum}

In this section we study the average of the exponential sum $H\left(a, m_{1}, m_{2} ; c\right)$ defined in (A.8). Recall that $\chi_{1}, \chi_{2}$ are primitive characters of conductor $D_{1}, D_{2}$. We again let $c_{1}=\left[c, D_{1}\right]$ and $c_{2}=\left[c, D_{2}\right]$. We study the average

$$
\begin{aligned}
R\left(m_{1}, m_{2}, n ; c\right) & :=\frac{c}{c_{1} c_{2}} \sum_{a(c)}^{*} e\left(\frac{n \bar{a}}{c}\right) H\left(a, m_{1}, m_{2} ; c\right) \\
& =\frac{c}{c_{1} c_{2}} \sum_{a(c)}^{*} e\left(\frac{n \bar{a}}{c}\right) \sum_{\substack{r_{1}\left(c_{1}\right) \\
r_{2}\left(c_{2}\right)}} \chi_{1}\left(r_{1}\right) \chi_{2}\left(r_{2}\right) e\left(\frac{m_{1} r_{1}}{c_{1}}+\frac{m_{2} r_{2}}{c_{2}}+\frac{a r_{1} r_{2}}{c}\right) .
\end{aligned}
$$

LEMMA B.1.

$$
\left|R\left(m_{1}, m_{2}, n ; c\right)\right| \leqslant\left(D_{1} D_{2}\right)^{3}\left(\frac{c_{1} c_{2}}{c^{2}} n-m_{1} m_{2}, c\right)
$$

Proof. For $p$ a fixed prime number, we define the exponents $d_{1}, d_{2}, k, k_{1}, k_{2}$ by $p^{d_{1}}\left\|D_{1}, p^{d_{2}}\right\| D_{2}, p^{k}\left\|c, p^{k_{1}}\right\| c_{1}, p^{k_{2}} \| c_{2}$. We set $c_{p}=c / p^{k}$, with $c_{1, p}$ and $c_{2, p}$ defined analogously. Let $\chi_{i, p}$ denote the $p$-primary component of $\chi_{i}$. By the Chinese Remainder Theorem we can factor $R\left(m_{1}, m_{2}, n ; c\right)$ over primes to get local sums (after some changes of variable) of the form

$$
\begin{aligned}
& p^{k-k_{1}-k_{2}} \sum_{a\left(p^{k}\right)}^{*} e\left(\frac{c_{1, p} c_{2, p}{\overline{c_{p}}}^{2} n \bar{a}}{p^{k}}\right) \sum_{r_{1}\left(p^{k_{1}}\right)} \chi_{1, p}\left(r_{1}\right) e\left(\frac{m_{1} r_{1}}{p^{k_{1}}}\right) \times \\
& \times \sum_{r_{2}\left(p^{k_{2}}\right)} \chi_{2, p}\left(r_{2}\right) e\left(\frac{m_{2} r_{2}}{p^{k_{2}}}+\frac{a r_{1} r_{2}}{p^{k}}\right) .
\end{aligned}
$$

Since the context is now clear, we henceforth write $\chi_{i}$ instead of $\chi_{i, p}$. We also set $D_{p}:=c_{1, p} c_{2, p} / c_{p}^{2}$. If $k<d_{1}+d_{2}$ the sum is trivially bounded by $p^{3\left(d_{1}+d_{2}\right)}$, so we may assume that $k \geqslant d_{1}+d_{2}$, so that $k_{1}=k_{2}=k$ and $D_{p}=c_{1} c_{2} / c^{2}$. We now break into cases.

B.0.1. The Case $p \times D_{1} D_{2}$

This is the generic case; the sum takes the form

$$
p^{-k} \sum_{a\left(p^{k}\right)}^{*} e\left(\frac{D_{p} n \bar{a}}{p^{k}}\right) \sum_{r_{1}\left(p^{k}\right)} e\left(\frac{m_{1} r_{1}}{p^{k}}\right) \sum_{r_{2}\left(p^{k}\right)} e\left(\frac{\left(m_{2}+a r_{1}\right) r_{2}}{p^{k}}\right)
$$


yielding the Ramanujan sum

$$
\sum_{a\left(p^{k}\right)}^{*} e\left(\frac{\left(D_{p} n-m_{1} m_{2}\right) \bar{a}}{p^{k}}\right) \ll\left(D_{p} n-m_{1} m_{2}, p^{k}\right)
$$

\section{B.0.2. The Case $p \nmid D_{1}, p \mid D_{2}$}

We have

$$
\begin{aligned}
& p^{-k} \sum_{a\left(p^{k}\right)}^{*} e\left(\frac{D_{p} n \bar{a}}{p^{k}}\right) \sum_{r_{1}\left(p^{k}\right)} \chi_{1}\left(r_{1}\right) e\left(\frac{m_{1} r_{1}}{p^{k}}\right) \sum_{r_{2}\left(p^{k}\right)} e\left(\frac{\left(m_{2}+a r_{1}\right) r_{2}}{p^{k}}\right) \\
& =\sum_{a\left(p^{k}\right)}^{*} e\left(\frac{\left(D_{p} n-m_{1} m_{2}\right) \bar{a}}{p^{k}}\right) \chi_{1}\left(-a \bar{m}_{2}\right) \ll p^{d_{1} / 2}\left(D_{p} n-m_{1} m_{2}, p^{k}\right),
\end{aligned}
$$

since this is a Gauss sum.

\section{B.0.3. The Case $p \mid D_{1}$ and $p \mid D_{2}$}

We have

$$
p^{-k} \sum_{a\left(p^{k}\right)}^{*} e\left(\frac{D_{p} n \bar{a}}{p^{k}}\right) \sum_{r_{1}\left(p^{k}\right)} \chi_{1}\left(r_{1}\right) e\left(\frac{m_{1} r_{1}}{p^{k}}\right) \sum_{r_{2}\left(p^{k}\right)} \chi_{2}\left(r_{2}\right) e\left(\frac{\left(m_{2}+a r_{1}\right) r_{2}}{p^{k}}\right) .
$$

The $r_{2}$ sum equals

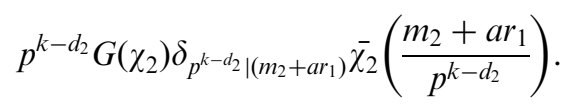

Since $k-d_{2} \geqslant d_{1}$ we may replace $\chi_{1}\left(r_{1}\right)$ by $\chi_{1}\left(-m_{2} \bar{a}\right)$ and the sum equals

$$
\begin{aligned}
& p^{2 k-d_{2}-k_{1}-k_{2}} G\left(\chi_{2}\right) \chi_{1}\left(-m_{2}\right) \sum_{a\left(p^{k}\right)}^{*} e\left(\frac{\left(D_{p} n-m_{1} m_{2}\right) \bar{a}}{p^{k}}\right) \chi_{1} \chi_{2}(\bar{a}) \sum_{s_{1}\left(p^{d_{2}}\right)} \overline{\chi_{2}}\left(s_{1}\right) e\left(\frac{m_{1} s_{1}}{p^{d_{2}}}\right) \\
& \quad \ll p^{\left(d_{1}+d_{2}\right) / 2}\left(D_{p} n-m_{1} m_{2}, p^{k}\right) .
\end{aligned}
$$

\section{B.1. THE CASE $\chi_{1}=\bar{\chi}_{2}$}

We now restrict to the particular case $\chi_{1}=\overline{\chi_{2}}=\chi$, with $D$, the conductor of $\chi$, square-free. We also suppose that $(n, D)=1$, which will always be the case in our applications. We have

$$
\begin{aligned}
R\left(m_{1}, m_{2}, n ; c\right)= & \frac{c}{[c, D]^{2}} \sum_{x(c)}^{*} e\left(\frac{n \bar{x}}{c}\right) \sum_{r_{1}, r_{2}([c, D])} \chi\left(r_{1}\right) \chi\left(\bar{r}_{2}\right) e\left(\frac{m_{1} r_{1}+m_{2} r_{2}}{[c, D]}+\frac{r_{1} r_{2} x}{c}\right) \\
= & \frac{c}{[c, D]^{2}} \frac{\varphi(c)}{\varphi([c, D])} \sum_{\substack{x, r_{1}, r_{2}([c, D]) \\
(x, c D)=1}} \chi\left(r_{1}\right) \bar{\chi}\left(r_{2}\right) \times \\
& \times e\left(\frac{m_{1} r_{1}+m_{2} r_{2}}{[c, D]}+\frac{r_{1} r_{2} x+n \bar{x}}{c}\right) .
\end{aligned}
$$


We factor the sum over primes, setting

$$
D=D_{1} D_{2} D_{3} \quad \text { and } \quad c=D_{1} c_{2} c_{3}
$$

with

$$
\left(c_{3}, D\right)=1, \quad D_{1}|| c, p\left|D_{2} \Rightarrow p^{2}\right| c_{2},\left(c_{3}, D\right)=1 .
$$

Then $[c, D]=c_{3} D_{1} c_{2} D_{3}$. We have

$$
R\left(m_{1}, m_{2}, n ; c\right)=R\left(m_{1}, m_{2}, n ; c_{3}\right) R\left(m_{1}, m_{2}, n ; c_{2}\right) R\left(m_{1}, m_{2}, n ; D_{1}\right) R\left(m_{1}, m_{2}, n ; D_{3}\right),
$$

where (after some simple changes of variable)

$$
\begin{aligned}
& R\left(m_{1}, m_{2}, n ; c_{3}\right)=\frac{1}{c_{3}} \sum_{x\left(c_{3}\right)}^{*} \sum_{r_{1}, r_{2}\left(c_{3}\right)} e\left(\frac{m_{1} r_{1}+m_{2} r_{2}+D_{3}\left(r_{1} r_{2} x+n \bar{x}\right)}{c_{3}}\right) \\
& R\left(m_{1}, m_{2}, n ; D_{3}\right)=\frac{1}{\varphi\left(D_{3}\right) D_{3}} \sum_{x\left(D_{3}\right)}^{*} \sum_{r_{1}, r_{2}\left(D_{3}\right)} \chi_{D_{3}}\left(r_{1}\right) \bar{\chi}_{D_{3}}\left(r_{2}\right) e\left(\frac{m_{1} r_{1}+m_{2} r_{2}}{D_{3}}\right) \\
& R\left(m_{1}, m_{2}, n ; c_{2}\right)=\frac{1}{c_{2}} \sum_{x\left(c_{2}\right)}^{*} \sum_{r_{1}, r_{2}\left(c_{2}\right)} \chi_{D_{2}}\left(r_{1}\right) \bar{\chi}_{D_{2}}\left(r_{2}\right) e\left(\frac{m_{1} r_{1}+m_{2} r_{2}+D_{3}\left(r_{1} r_{2} x+n \bar{x}\right)}{c_{2}}\right), \\
& R\left(m_{1}, m_{2}, n ; D_{1}\right) \\
& \quad=\frac{1}{D_{1}} \sum_{x\left(D_{1}\right)}^{*} \sum_{r_{1}, r_{2}\left(D_{1}\right)} \chi_{D_{1}}\left(r_{1}\right) \bar{\chi}_{D_{1}}\left(r_{2}\right) e\left(\frac{m_{1} r_{1}+m_{2} r_{2}+D_{3}\left(r_{1} r_{2} x+n \bar{x}\right)}{D_{1}}\right) .
\end{aligned}
$$

To evaluate the first sum, we sum first over $r_{1}$, getting

$$
R\left(m_{1}, m_{2}, n ; c_{3}\right)=r\left(m_{1} m_{1}-D_{3}^{2} n ; c_{3}\right)=r\left(m_{1} m_{1}-(D /(c, D))^{2} n ; c_{3}\right) .
$$

The second sum can also be evaluated by doing the $r_{1}$ sum first, getting

$$
R\left(m_{1}, m_{2}, n ; D_{3}\right)=\chi_{D_{3}}(-1) \bar{\chi}_{D_{3}}\left(m_{1}\right) \chi_{D_{3}}\left(m_{2}\right) .
$$

The third and fourth sums are slightly more complex. Since they are multiplicative in their modulus, we can factor over primes, reducing to the evaluation of

$$
R\left(m_{1}, m_{2}, n ; p^{\alpha}\right)=\frac{1}{p^{\alpha}} \sum_{x\left(p^{\alpha}\right)}^{*} \sum_{r_{1}, r_{2}\left(p^{\alpha}\right)} \chi_{p}\left(r_{1}\right) \bar{\chi}_{p}\left(r_{2}\right) e\left(\frac{m_{1} r_{1}+m_{2} r_{2}+D_{3}\left(r_{1} r_{2} x+n \bar{x}\right)}{p^{\alpha}}\right) .
$$

\section{B.1.1. The Third Sum $(\alpha>1)$}

First note that if $p \mid m_{1} m_{2}$, then $R\left(m_{1}, m_{2}, n ; p^{\alpha}\right)=0$, as can be seen by summing over $r_{i}$ if $p \mid m_{i}$. Thus we may assume that $p \nmid m_{1} m_{2}$, so we get

$$
R\left(m_{1}, m_{2}, n ; p^{\alpha}\right)=\bar{\chi}_{p}\left(m_{1}\right) \chi_{p}\left(m_{2}\right) r\left(1,1, \overline{m_{1} m_{2}} n ; p^{\alpha}\right) .
$$


Next we set $r_{1}=x_{1}+p y_{1}$ with $x_{1}<p \quad y_{1} \bmod \left(p^{\alpha-1}\right)$. Summing over $y_{1}$ we get $1+r_{2} x \equiv 0\left(p^{\alpha-1}\right)$. Letting $1+r_{2} x=z p^{\alpha-1}$, we sum over $x_{1}$, then over $z$, getting Gauss sums, and finally over $x$, getting

$$
R\left(m_{1}, m_{2}, n ; p^{\alpha}\right)=\bar{\chi}_{p}\left(m_{1}\right) \chi_{p}\left(m_{2}\right) r\left(m_{1} m_{2}-D_{3}^{2} n ; p^{\alpha}\right) .
$$

\section{B.1.2. The Fourth Sum $(\alpha=1)$.}

Note that if $p \mid m_{1} m_{2}$,

$$
R\left(m_{1}, m_{2}, n ; p\right)= \begin{cases}\chi^{2}\left(m_{2}\right) \bar{\chi}_{p}\left(m_{1} m_{2}-n D_{3}^{2}\right) G\left(\bar{\chi}_{p}^{2}\right) & \text { if } p \mid m_{1} \\ \bar{\chi}^{2}\left(m_{1}\right) \chi_{p}\left(m_{1} m_{2}-n D_{3}^{2}\right) G\left(\chi_{p}^{2}\right) & \text { if } p \mid m_{2}\end{cases}
$$

When $\left(p, m_{1} m_{2}\right)=1$ we have $R\left(m_{1}, m_{2}, n ; p\right)=\bar{\chi}_{p}\left(m_{1}\right) \chi_{p}\left(m_{2}\right) R\left(1,1, n \overline{m_{1} m_{2}} ; p\right)$ and some simple computations then give

$$
R\left(m_{1}, m_{2}, n ; p\right)=\chi_{p}(-1) \bar{\chi}_{p}\left(m_{1}\right) \chi_{p}\left(m_{2}\right) \sum_{x(p)} \bar{\chi}_{p}\left(x\left(1+n D_{3}^{2} \overline{m_{1} m_{2}} x\right)\right) \chi_{p}(1+x) .
$$

An interesting case occurs when $m_{1} m_{2}=n D_{3}^{2}$, in which case

$$
R\left(m_{1}, m_{2}, n ; p\right)=-\bar{\chi}_{p}\left(m_{1}\right) \chi_{p}\left(m_{2}\right) .
$$

This is the source of the 'off-diagonal' term in the main text.

To summarize, we have

$$
R\left(m_{1}, m_{2}, n ; c\right)=\chi_{D_{3}}(-1) r\left(m_{1} m_{2}-D_{3}^{2} n ; c^{\prime}\right) \bar{\chi}_{D_{2} D_{3}}\left(m_{1}\right) \chi_{D_{2} D_{3}}\left(m_{2}\right) R\left(m_{1}, m_{2}, n ; D_{1}\right)
$$

where the variables are defined by

$$
D=D_{1} D_{2} D_{3}, \quad c=c^{\prime} D_{1}, \quad\left(c^{\prime}, D_{1} D_{3}\right)=1, D_{2}^{2} \mid c^{\prime} .
$$

\section{B.2. THE CASE $\chi_{1}=1$}

Finally, we consider the case when $\chi_{1}$ is trivial, so $D_{1}=1$ and $\left[c, D_{1}\right]=c$. To simplify notation, we use $\chi$ rather than $\chi_{2}$ to denote the non-trivial character. We wish to sum

$$
R\left(m_{1}, m_{2}, n ; c\right)=\frac{1}{[c, D]} \sum_{a(c)}^{*} e\left(\frac{n \bar{a}}{c}\right) \sum_{\substack{r_{1}(c) \\ r_{2}([c, D])}} \chi\left(r_{2}\right) e\left(\frac{m_{1} r_{1}+a r_{1} r_{2}}{c}+\frac{m_{2} r_{2}}{[c, D]}\right) .
$$

Performing the $r_{1}$ sum gives

$$
\frac{c}{[c, D]} \sum_{a(c)}^{*} e\left(\frac{n \bar{a}}{c}\right) \sum_{r_{2}([c, D])} \delta_{m_{1}+a r_{2} \equiv 0(c)} \chi\left(r_{2}\right) e\left(\frac{m_{2} r_{2}}{[c, D]}\right) .
$$

Since $D$ is squarefree, we can write $[c, D]=c D_{2}$, with $D_{2}=D /(c, D)$ and $\left(D_{2}, c\right)=1$. We factor $\chi=\chi_{1} \chi_{2}$ such that the conductor of $\chi_{2}$ is $D_{2}$. We split $r_{2}$ over relatively prime moduli as $r_{2}=s_{1} c+s_{2} D_{2}$ to make the sum 


$$
\frac{1}{D_{2}} \sum_{a(c)}^{*} e\left(\frac{n \bar{a}}{c}\right) \sum_{s_{1}<D_{2}} \sum_{s_{2}(c)} \delta_{m_{1}+a D_{2} s_{2} \equiv 0(c)} \chi_{1}\left(s_{2} D_{2}\right) \chi_{2}\left(s_{1} c\right) e\left(\frac{m_{2} s_{1}}{D_{2}}+\frac{m_{2} s_{2}}{c}\right) .
$$

Note that the $s_{2}$ sum only contributes one term, $s_{2}=-m_{1} \bar{a} \overline{D_{2}}$. The $s_{1}$ sum is a Gauss sum, so the entire expression is

$$
\frac{G\left(\chi_{2}\right)}{D_{2}} \chi_{2}\left(c \bar{m}_{2}\right) \chi_{1}\left(-m_{1}\right) \sum_{a(c)}^{*} e\left(\frac{\left(n-m_{1} m_{2} \overline{D_{2}}\right) \bar{a}}{c}\right) \chi_{1}(\bar{a}) .
$$

\section{Acknowledgements}

This paper was written while the first author enjoyed the hospitality of the Institute for Advanced Study during the 1999-2000 academic year on the occasion of the program 'Analytic Number Theory and Automorphic $L$-functions' and during the fall 2000 on the occasion of the program 'Automorphic Forms and Representation Theory'. This paper is the last of a series of three papers ([KMV2, KMV3]) studying the second moment of Rankin-Selberg convolution $L$-functions of the elements of $S_{2}^{*}(q)$ against a fixed modular form. During the course of this project we have benefitted greatly from the encouragement and guidance of Henryk Iwaniec and Peter Sarnak, and it is a pleasure to thank them as we reach the end.

\section{References}

[BFH] Bump, D., Friedberg, S. and Hoffstein, J.: Nonvanishing theorems for $L$-functions of modular forms and their derivatives, Invent. Math. 102(3) (1990), 543-618.

[CF] Conrey, B. and Farmer, D.: Mean values of $L$-functions and symmetry, Internat. Math. Res. Notices 17 (2000), 883-908.

[Da] Davenport, H.: Multiplicative Number Theory, Grad. Texts in Math. 74, SpringerVerlag, New York, 1980.

[Du] Duke, W.: The critical order of vanishing of automorphic $L$-functions with large level, Invent. Math. 119(1) (1995), 165-174.

[DFI1] Duke, W., Friedlander, J. and Iwaniec, H.: A quadratic divisor problem, Invent. Math. 115 (1994), 219-239.

[DFI2] Duke, W., Friedlander, J. and Iwaniec, H.: Bounds for automorphic $L$-functions. II, Invent. Math. 115 (1994).

[EMOT] Erdeilyi, A., Magnus, W., Oberhettinger, F. and Tricomi, F. G.: Tables of Integral Transforms. Vol. I, II., Based, in part, on notes left by Harry Bateman, McGrawHill, New York, 1954.

[GZ] Gross, W. and Zagier, D.: Heegner points and derivatives of $L$-series, Invent. Math. 84(2) (1986), 225-320.

[I] Iwaniec, H.: Topics in Classical Automorphic Forms, Grad. Stud. Math. 17, Amer. Math. Soc., Providence, RI, 1997.

[IS] Iwaniec, H. and Sarnak, P.: The non-vanishing of central values of automorphic $L$-functions and Landau-Siegel zeros, Israel J. Math. 120 (2000), part A, $155-177$.

[J] Jutila, M.: A Method in the Theory of Exponential Sums, Tata Lecture Notes Math. 80, Bombay (1987). 
[KeSn] Keating, J. and Snaith, N. Comm. Math. Phys. 214 (2000), 57-89 and 91-110.

[Ko] Kolyvagin, V. Euler Systems. The Grothendieck Festschrift, Vol. II, Progr. in Math. 87, Birkhauser, Boston, 1990, pp. 435-483.

[KL] Kolyvagin, V. and Logachev.: Finiteness of the Shafarevich-Tate group and the group of rational points for some modular abelian varieties, Leningrad Math. J. 1(5) (1990), 1229-1253.

[KM1] Kowalski, E. and Michel, P.: The analytic rank of $J_{0}(q)$ and zeros of automorphic L-functions, Duke Math. J. 100 (1999), 503-547.

[KM2] Kowalski, E. and Michel, P.: A lower bound for the rank of $J_{0}(q)$, Acta Arith. 94(4) (2000), 303-343.

[KMV1] Kowalski, E., Michel, P. and VanderKam, J.: Non-vanishing of high derivatives of automorphic $L$-functions at the center of the critical strip, J. Reine Angew. Math. 526 (2000), 1-34.

[KMV2] Kowalski, E., Michel, P. and VanderKam J.: Mollification of the fourth moment of automorphic $L$-functions and arithmetic applications, Invent. Math. 142(1) (2000), 95-151.

[KMV3] Kowalski, E., Michel, P. and VanderKam, J.: Rankin-Selberg $L$-functions in the level aspect, Duke Math. J. 114(1) (2002), 123-191.

[KS] Katz, N. M. and Sarnak, P.: Zeroes of zeta functions and symmetry, Bull. Amer. Math. Soc. (NS) 36(1) (1999), 1-26.

[La] Langlands, R. P.: Base Change for GL(2), Ann. Math. Stud., Princeton Univ. Press, Princeton, NJ, 1980.

[Li] Li, W.: Newforms and functional equations, Math. Ann. 212 (1975), 285-315.

[Lu1] Luo, W.: On the nonvanishing of Rankin-Selberg L-functions, Duke Math. J. 69(2) (1993), 411-425.

[Lu2] Luo, W.: Nonvanishing of $L$ values and the strong Weyl law, Preprint, 2000.

[Me] Merel, L.: Sur la nature non-cyclotomique des points d'ordre fini des courbes elliptiques, Duke Math. J. 110(1) (2001), 81-119.

[MM] Murty, V. K. and Murty, M. R.: Mean values of derivatives of modular $L$-series, Ann. of Math. (2) 133(3) (1991), 447-475.

[PS] Phillips, R. and Sarnak, P.: On cusp forms for co-finite subgroups of $\operatorname{PSL}(2, R)$, Invent. Math. 80(2) (1985), 339-364.

[Ru] Rubin, K.: Euler systems and modular elliptic curves, In: Galois Representations in Arithmetic Algebraic Geometry (Durham, 1996), London Math. Soc. Lecture Note Ser. 254, Cambridge Univ. Press, Cambridge, 1998, pp. 351-367.

[Ro] Royer, E.: Statistique de la variable L( $\left.\operatorname{sym}^{2} f, 1\right)$, Math. Ann. 321 (2001), 667-687.

[Sc] Scholl, A.: An introduction to Kato's Euler systems, Galois Representations in Arithmetic Algebraic Geometry (Durham, 1996), London Math. Soc. Lecture Note Ser. 254, Cambridge Univ. Press, Cambridge, 1998, pp. 379-460.

[Sh] Shimura, G.: On the periods of modular forms, Math. Ann. 229(3) (1977), 211221.

[V1] VanderKam, J.: The rank of quotients of $J_{0}(N)$, Duke Math. J. 97(3) (1999), 545577.

[V2] VanderKam, J.: Linear independence of Hecke operators in the homology of $X_{0}(N)$, J. London Math. Soc. 61(2) (2000), 349-358. 